Key Words:

Components-in-Grout

Porflow

Retention:

Permanent

\title{
SPECIAL ANALYSIS: UPDATE OF DISPOSAL OF CEMENT-STABILIZED ENCAPSULATED WASTE AT THE E-AREA LOW-LEVEL WASTE FACILITY
}

Prepared by:

Leonard B. Collard

James R. Cook

May 21, 2003

Westinghouse Savannah River Company

Savannah River Site

Aiken, SC 29808

Prepared for the U.S. Department of Energy Under

Contract Number DE-AC09-96SR18500 
This document was prepared in conjunction with work accomplished under Contract No. DE-AC09-96SR18500 with the U. S. Department of Energy.

\section{DISCLAIMER}

This report was prepared as an account of work sponsored by an agency of the United States Government. Neither the United States Government nor any agency thereof, nor any of their employees, makes any warranty, express or implied, or assumes any legal liability or responsibility for the accuracy, completeness, or usefulness of any information, apparatus, product or process disclosed, or represents that its use would not infringe privately owned rights. Reference herein to any specific commercial product, process or service by trade name, trademark, manufacturer, or otherwise does not necessarily constitute or imply its endorsement, recommendation, or favoring by the United States Government or any agency thereof. The views and opinions of authors expressed herein do not necessarily state or reflect those of the United States Government or any agency thereof.

This report has been reproduced directly from the best available copy.

Available for sale to the public, in paper, from: U.S. Department of Commerce, National Technical Information Service, 5285 Port Royal Road, Springfield, VA 22161, phone: (800) 553-6847, fax: (703) 605-6900

email: orders@ntis.fedworld.gov

online ordering: http://www.ntis.gov/help/index.asp

Available electronically at http://www.osti.gov/bridge

Available for a processing fee to U.S. Department of Energy and its contractors, in paper, from: U.S. Department of Energy, Office of Scientific and Technical Information, P.O. Box 62, Oak Ridge, TN 37831-0062,

phone: (865)576-8401,

fax: (865)576-5728

email: $\underline{\text { reports@ adonis.osti.gov }}$ 
Key Words:

Components-in-Grout

Porflow

Retention:

Permanent

\section{SPECIAL ANALYSIS: UPDATE OF DISPOSAL OF CEMENT-STABILIZED ENCAPSULATED WASTE AT THE E-AREA LOW-LEVEL WASTE FACILITY}

Prepared by:

Leonard B. Collard

James R. Cook

May 21, 2003

Westinghouse Savannah River Company

Savannah River Site

Aiken, SC 29808

Prepared for the U.S. Department of Energy Under

Contract Number DE-AC09-96SR18500

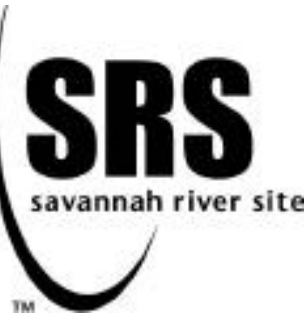




\section{TABLE OF CONTENTS}

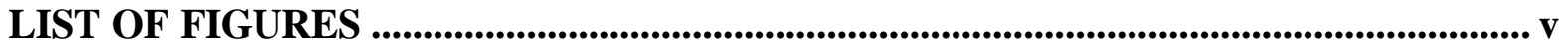

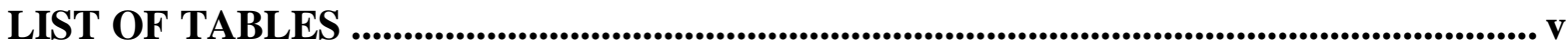

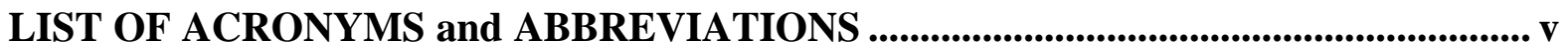

1.0 EXECUTIVE SUMMARY ........................................................................................... 1

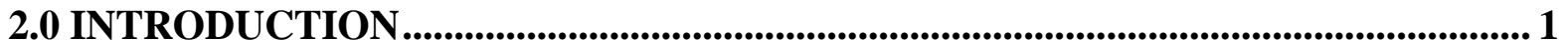

3.0 GROUNDWATER PATHWAY ASSESSMENT .................................................... 2

3.1 Modeling Changes and Improvements ...................................................................... 2

3.1.1 Vadose zone modeling changes and improvements ............................................ 3

3.1.2 Aquifer modeling changes and improvements ........................................................ 6

3.2 Groundwater Pathway Results...................................................................................... 7

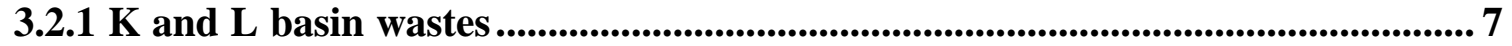

3.2.2 High-concentration I-129 waste ..................................................................... 7

3.2.3 ETF activated carbon vessels ..............................................................................9

4.0 INTRUDER AND AIR PATHWAY ANALYSIS ...................................................9

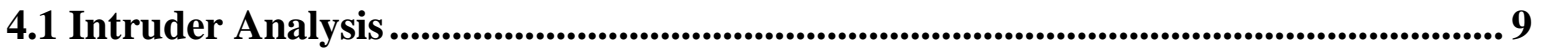

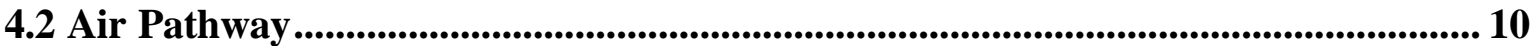

5.0 CONCLUSIONS ........................................................................................................................ 11

5.1 Comparison of Old and Revised Inventory Limits.............................................. 11

5.2 Inventory and Volume Consumption for $K$ and $L$ Basin Resins.............................. 13

5.3 ETF Activated Carbon Inventory and Volume Consumption................................... 14

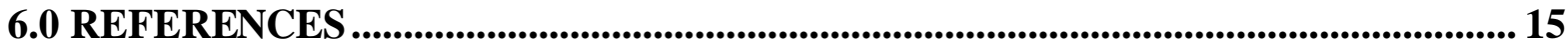

6.1 Groundwater Pathway References ..................................................................... 15

6.2 Intruder and Air Pathway References............................................................ 16

Appendix A. PA Replacement Tables................................................................................................ 17

Appendix B. PA Replacement Figures....................................................................................... 38

Appendix C. Air Analysis Report .................................................................................................... 52

Appendix D. Groundwater Pathway Model QA Changes .............................................. 58

Appendix E. Design Check........................................................................................................6 60 


\section{LIST OF FIGURES}

Figure 1. Components-in-Grout Vadose Zone Models: PA on left, New Model on Right ...... 3

Figure 2. Inventory limits versus Kd for high-concentration I-129 waste as CIG ................. 9

Figure 3. Conceptual model for fixed concentration test case .......................................... 58

Figure 4 Concentration histories for fixed concentration test case ................................... 58

\section{LIST OF TABLES}

Table 1. Changes in saturated hydraulic conductivity ...................................................... 4

Table 2. Comparison of fractions for solubility-limited radionuclide ............................... 5

Table 3. Resin-Specific Calculated $K_{d}$ Values and Generic PA $K_{d}$ Values.......................... 5

Table 4. Aquifer source node locations ......................................................................... 6

Table 5. Effect of measuring waste-specific Kds ........................................................ 7

Table 6. Groundwater pathway results for high-concentration I-129 wastes ...................... 8

Table 7. Comparison of old and revised inventory limits.............................................. 12

Table 8. Current and projected inventory for $\mathrm{K}$ and $\mathrm{L}$ basin compared to limits ................. 14

Table 9. ETF activated carbon vessel inventory and facility volume consumption............... 14

$\begin{array}{ll}\mu \mathbf{C i} & \text { microcuries } \\ \mathbf{C i} & \text { curie } \\ \mathbf{D O E} & \text { U.S. Department of Energy } \\ \mathbf{E T F} & \text { Effluent Treatment Facility } \\ \mathbf{f t} & \text { feet } \\ \mathbf{g} & \text { gram } \\ \mathbf{K} & \text { sorption coefficient } \\ \mathbf{L} & \text { liters } \\ \mathbf{L o g} & \text { logarithm } \\ \mathbf{m} & \text { meters } \\ \mathbf{M C L} & \text { maximum contaminant level } \\ \mathbf{m l} & \text { milliliters } \\ \mathbf{P A} & \text { performance assessment } \\ \mathbf{p C i} & \text { picocuries } \\ \mathbf{S A} & \text { Special Analysis } \\ \text { UDQ } & \text { Unreviewed Disposal Question }\end{array}$

\section{LIST OF ACRONYMS AND ABBREVIATIONS}

\section{microcuries}

curie

U.S. Department of Energy

Effluent Treatment Facility

gram

sorption coefficien

liters

logarithm

maximum contaminant level

milliliters

performance assessment

Special Analysis

Unreviewed Disposal Question 


\subsection{EXECUTIVE SUMMARY}

This Special Analysis for Components-in-Grout (CIG) expands the list of isotopes beyond that considered in Revision 0 (Cook, et al., 1999) to the full suite of normal isotopes. This revision also addresses selected isotopes in special waste forms from the $\mathrm{K}$ and $\mathrm{L}$ basin resins that have waste-specific Kds and high-concentration I-129 wastes with waste-specific Kds, including Effluent Treatment Facility (ETF) activated carbon vessels.

The full suite of normal isotopes was first screened using the Slit Trench screening results as a conservative approach. The isotopes that survived the screening were analyzed to determine the appropriate CIG inventory limits.

The groundwater modeling was revised to incorporate improvements and changes in other recent Special Analyses and Unreviewed Disposal Question (UDQ) evaluations. Those reports include the following:

- Analysis of aquifer source node location (Flach and Collard, 2003)

- Correction of E-Area disposal limits (Cook, 2002)

- Variations in trench dimensions (Cook, 2003)

- UDQ evaluation for K and L basin resins (Collard, 2003)

- Waste-specific Kd values for K and L basin resins (Kaplan and Coffee, 2002)

- UDQ evaluation for ETF activated carbon vessels (Collard, 2002)

- Backfill soil compaction requirements (Phifer and Collard, 2003)

The air pathway analysis was modified to consider a distributed source rather than a point source. These changes are discussed below in intruder and groundwater sections.

Tables and figures are provided in appendices to replace all the tables and figures in the Performance Assessment report that are directly related to the most recent analyses. .

Changes to inventory limits are shown in Table 7. Inventory limits for solubility-limited radionuclides require special treatment as discussed in Section 3.1.1.3. U-238 and Pu-239 were analyzed as being solubility-limited, because otherwise they would consume excessive amounts of their inventory limits. Other $\mathrm{U}$ and $\mathrm{Pu}$ isotopes were not analyzed as being solubility-limited because they would not consume excessive amounts of inventory limits.

Current and projected inventories for the $\mathrm{K}$ and $\mathrm{L}$ basin resins are compared against inventory limits for a single set of 5 CIG trenches (see Table 8). Projections for the K and L basin waste are through 2035, thus actual inventory consumption is dependent on the total number of CIG trenches excavated and filled through 2035. Current inventory for three ETF activated carbon vessels awaiting disposal are compared against inventory limits for a single set of 5 CIG trenches (see Table 9).

\subsection{INTRODUCTION}

This Special Analysis for Components-in-Grout (CIG) expands the list of isotopes beyond that considered in Revision 0 (Cook, et al., 1999) to the full suite of normal isotopes. This 
revision also addresses selected isotopes in special waste forms from the $\mathrm{K}$ and $\mathrm{L}$ basin resins that have waste-specific Kds and high-concentration I-129 wastes with waste-specific Kds, including Effluent Treatment Facility (ETF) activated carbon vessels.

The full suite of normal isotopes was first screened using the Slit Trench screening results as a conservative approach. The isotopes that survived the screening were analyzed to determine the appropriate CIG inventory limits.

The groundwater modeling was revised to incorporate improvements and changes in other recent Special Analyses and Unreviewed Disposal Question (UDQ) evaluations. Those reports include the following:

- Analysis of aquifer source node location (Flach and Collard, 2003)

- Correction of E-Area disposal limits (Cook, 2002)

- Variations in trench dimensions (Cook, 2003)

- UDQ evaluation for K and L basin resins (Collard, 2003)

- Waste-specific Kd values for K and L basin resins (Kaplan and Coffee, 2002)

- UDQ evaluation for ETF activated carbon vessels (Collard, 2002)

- Backfill soil compaction requirements (Phifer and Collard, 2003)

The air pathway analysis was modified to consider a distributed source rather than a point source. These changes are discussed below in intruder and groundwater sections.

Tables and figures are provided in appendices to replace all the tables and figures in the Performance Assessment report that are directly related to the most recent analyses.

Changes to inventory limits are presented. $\mathrm{K}$ and $\mathrm{L}$ basin resins and three ETF activated carbon vessel wastes are explicitly examined.

\subsection{GROUNDWATER PATHWAY ASSESSMENT}

\subsection{Modeling Changes and Improvements}

Several modeling changes and improvements were incorporated in the current Special Analysis. The categories of modeling changes can be separated into vadose zone models and aquifer models as follows:

\section{$\underline{\text { Vadose zone model }}$}

- Selection of material types

- Changes in saturated hydraulic conductivity

- Solubility limits for U and Pu are not incorporated except for U-238 and Pu-239

- Increased sampling frequency for contaminant flux at water table after 300 years

- $\mathrm{K}$ and $\mathrm{L}$ basin resin waste

- High-concentration I-129 waste 
Aquifer model

- Increased sampling frequency for well concentrations

- Aquifer source node location

- Reduced size of model

- High-concentration I-129 waste

Each change and improvement is described below.

\subsubsection{Vadose zone modeling changes and improvements}

\subsubsection{Selection of material types}

The computer model was revised to better represent current disposal operations. The material types for the waste zone and the surrounding grout layer were revised.

The original PA model had a trench that was $20 \mathrm{ft}$ wide by $20 \mathrm{ft}$ tall (see Figure 1). The top five feet of the trench consisted of clean backfill. The waste was placed on a 1 - $\mathrm{ft}$ thick grout layer that covered the entire width of the trench. The waste was 5 feet thick by 18 feet wide with 1-ft thick grout vertical walls surrounding it. A 9- $\mathrm{ft}$ thick grout layer covered the waste.

The revised model used in this study has the same overall dimensions as the PA model (see Figure 1). However, only the top four feet of the trench consists of clean backfill. The waste was placed on a 1-ft thick grout layer that covered the entire width of the trench. The waste was 14 feet thick by 18 feet wide with $1-\mathrm{ft}$ thick grout vertical walls surrounding it. A 1 - $\mathrm{ft}$ thick grout layer covered the waste.
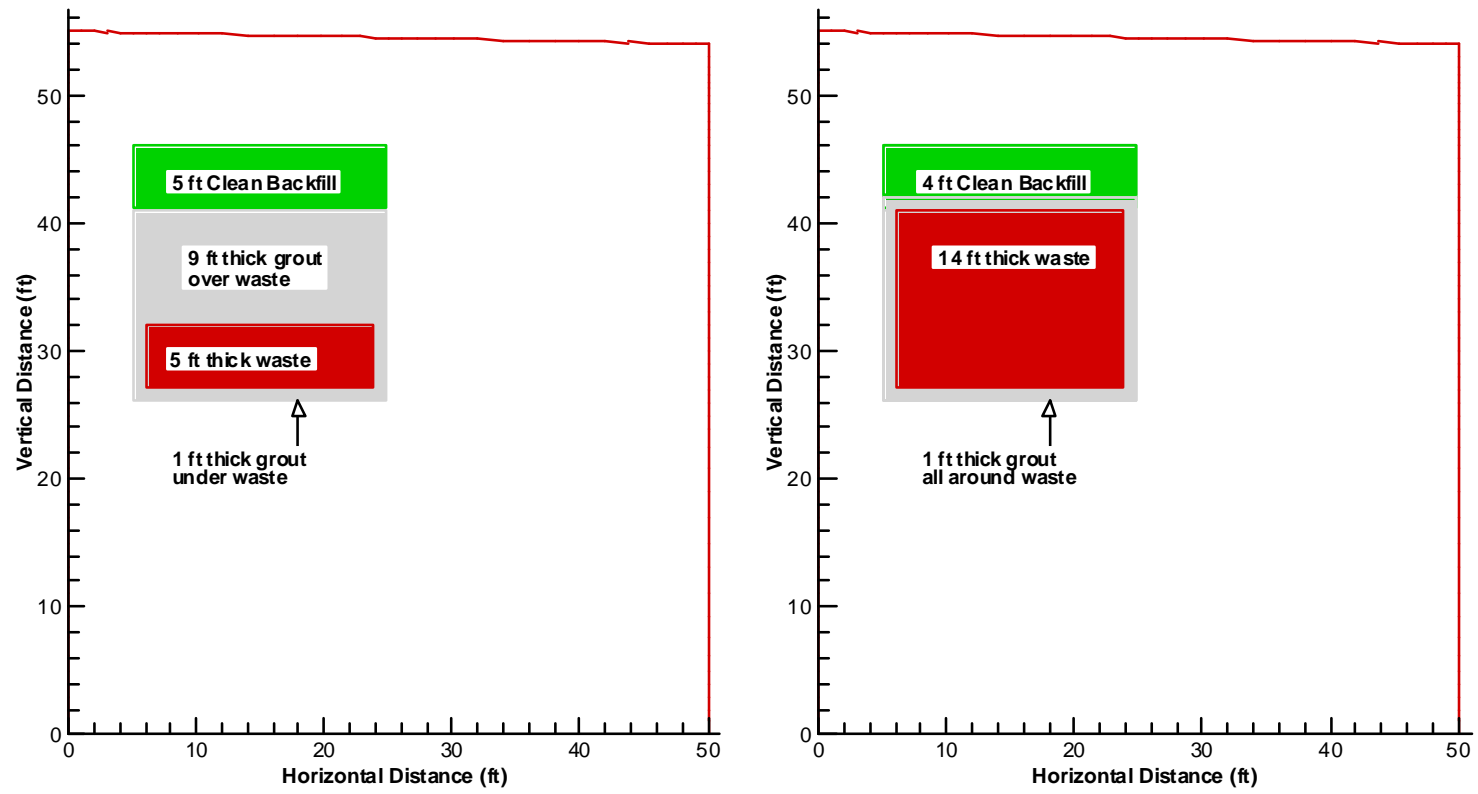

Figure 1. Components-in-Grout Vadose Zone Models: PA on left, New Model on Right 


\subsubsection{Changes in saturated hydraulic conduct ivity}

The changes in the saturated hydraulic conductivity are shown in Table 1. In the new model the saturated hydraulic conductivity for backfill materials was set to $1 \mathrm{E}-4 \mathrm{~cm} / \mathrm{sec}$ for the first 300 years to better represent field conditions (see Phifer and Collard, 2003). After 300 years the value was increased to $1 \mathrm{E}-3 \mathrm{~cm} / \mathrm{sec}$ to agree with Table C.1-9 in the PA.

Table 1. Changes in saturated hydraulic conductivity

\begin{tabular}{llclc}
\hline & \multicolumn{2}{c}{$\begin{array}{c}\text { Saturated Hydraulic Conductivity } \\
(\mathrm{cm} / \mathrm{sec})\end{array}$} & $\begin{array}{c}\text { So0+ years } \\
\text { Saturated Hydraulic Conductivity } \\
(\mathrm{cm} / \mathrm{sec})\end{array}$ \\
Material Type & PA & SA & PA & SA \\
\hline Clean Backfill & 1E-5 & $1 \mathrm{E}-4$ & $1 \mathrm{E}-5$ & $1 \mathrm{E}-3$ \\
Backfill & $1 \mathrm{E}-6$ & $1 \mathrm{E}-4$ & $1 \mathrm{E}-5$ & $1 \mathrm{E}-3$ \\
Waste & $1 \mathrm{E}-8$ & $1 \mathrm{E}-5$ & $1 \mathrm{E}-5$ & $1 \mathrm{E}-3$ \\
\hline
\end{tabular}

\subsubsection{Solubility limits for $U$ and $P u$ not incorporated with two exceptions}

The Kds for $\mathrm{U}$ and $\mathrm{Pu}$ in the waste zone are high values, because sufficient Fe corrosion products are assumed to exist in the waste zone to provide sorption surfaces. To reduce the analysis time, the additional benefit from solubility limits was not included for this Special Analysis, except for two radionuclides, namely U-238 and Pu-239.

For solubility-limited radionuclides, inventory limits for the groundwater pathway are not directly applicable. The analyses were performed by fixing the contaminant concentration in the waste zone at the solubility limit for the entire simulation. The initial inventory in 10 trenches required to produce this concentration (assuming 10 uniformly filled trenches each $200 \mathrm{~m}$ long) was 5.0E-3 Ci for U-238 and 4.6E-1 Ci for Pu-239. Any inventory equal to or greater than these amounts will produce the same well concentrations. For these radionuclides the fraction as part of the sum-of-fractions is more appropriately calculated as the peak well concentration divided by its MCL. The worst-case fractions for U-238 and $\mathrm{Pu}-239$ are 5.88E-06 and 6.75E-07, respectively.

If the worst-case fraction for the groundwater pathway is always less than or equal to the fraction for some other pathway, then the groundwater pathway can never be the most restrictive pathway, in which case the groundwater pathway can be ignored. Fraction comparisons at the minimum inventory that maintains the concentration at the solubility limit in the waste zone (the minimum solubility-limiting inventory) indicate whether the groundwater pathway can be ignored. If the fraction for some other pathway is less than the fraction for the groundwater pathway at the minimum solubility-limiting inventory, then the fraction for the other pathway will always be less than or equal to the fraction for the groundwater pathway. For smaller inventories both pathway fractions increase linearly with respect to the inventory, thus the fraction for the other pathway must always be greater. For larger inventories, the fraction for the other pathway increases linearly with respect to the inventory, but the fraction for the groundwater pathway remains fixed, thus the fraction for the other pathway must always be greater. 
For U-238 and $\mathrm{Pu}-239$ the fractions for the agriculture scenario and the groundwater pathway are shown in Table 2. For both nuclides the agriculture scenario fraction is greater than the groundwater pathway fraction, thus that the groundwater pathway fraction is always less than the fraction for the agriculture scenario and the fraction from the groundwater pathway can be ignored.

Table 2. Comparison of fractions for solubility-limited radionuclide

\begin{tabular}{lllll}
\hline Radionuclide & $\begin{array}{l}\text { Minimum } \\
\text { inventory to } \\
\text { invoke solubility } \\
\text { control (Ci) }\end{array}$ & $\begin{array}{l}\text { Groundwater } \\
\text { pathway fraction } \\
\text { (worst case) }\end{array}$ & $\begin{array}{l}\text { Agriculture } \\
\text { scenario } \\
\text { inventory limit } \\
(\mathrm{Ci})\end{array}$ & $\begin{array}{l}\text { Agriculture } \\
\text { scenario fraction }\end{array}$ \\
\hline $\mathrm{U}-238$ & $5.0 \mathrm{E}-3$ & $5.88 \mathrm{E}-6$ & 120 & $4.17 \mathrm{E}-5$ \\
$\mathrm{Pu}-239$ & $4.6 \mathrm{E}-1$ & $6.75 \mathrm{E}-7$ & 130 & $3.54 \mathrm{E}-3$ \\
\hline
\end{tabular}

\subsubsection{Increased sampling frequency for contaminant flux at water table after 300 years}

The sampling frequency for contaminant flux at the water table was increased after 300 years to more closely capture the peak for contaminants that are highly mobile, such as for I-129 that peaked at 390 years in the PA. The PA sampled every 10 years, but for this SA the sampling was increased to every year.

\subsubsection{K and L basin resin waste}

The $\mathrm{K}$ and $\mathrm{L}$ basin resin waste contains three contaminants for which special Kds were assigned (Kaplan and Coffee, 2002) as shown in Table 3. The cement-leachate values are used in this report. For the generic waste, the PA used values for corrosion products assuming that sufficient iron-based metals were available to provide adsorption surfaces for all the waste. The waste-specific Kds are substantially higher than the Kds for generic waste.

\section{Table 3. Resin-Specific Calculated $K_{d}$ Values and Generic PA $K_{d}$ Values}

\begin{tabular}{|c|c|c|c|}
\hline & \multicolumn{2}{|c|}{ Resin-Specific } & Generic PA \\
\hline & $\begin{array}{c}\text { Acid-Rain } \\
\text { Leachate } \\
(\mathrm{ml} / \mathrm{g})\end{array}$ & $\begin{array}{c}\text { Cement } \\
\text { Leachate } \\
(\mathrm{ml} / \mathrm{g})\end{array}$ & $\begin{array}{c}\text { Corrosion } \\
\text { Product Waste } \\
(\mathrm{ml} / \mathrm{g})\end{array}$ \\
\hline C-14 & 240 & 140 & 2 \\
\hline Tc-99 & $>680$ & $>810$ & 0.36 \\
\hline I- $129^{1}$ & $>3700$ & $>3700$ & 0.6 \\
\hline
\end{tabular}

${ }^{1}$ Provisional values - I-129 in the waste was below detection limits. Values were estimated based on measurements for other spent resins (Kaplan and Coffee, 2000)

\subsubsection{High-Concentration I-129 waste}

A suite of high-concentration I-129 wastes was analyzed for possible disposal as CIG. An equation was generated relating I-129 inventory limits to Kds. That equation is provided in 
the results section. Results for the high-concentration I-129 wastes were combined with generic I- 129 waste, the $\mathrm{K}$ and L basin waste, and a hypothetical waste with a $\mathrm{Kd}$ of 10000 $\mathrm{ml} / \mathrm{g}$ to provide a wide range of application for the equation.

\subsubsection{Aquifer modeling changes and improvements}

\subsubsection{Increased sampling frequency for well concentrations}

Similar to the increased sampling frequency in the vadose zone models, the sampling frequency was also increased for the well concentrations in the aquifer model.

\subsubsection{Aquifer source node location}

The aquifer source node (cell) locations and the total volume that initially diluted all contaminant fluxes changed as shown in Table 4. The I, J and $\mathrm{K}$ indices refer to the $\mathrm{X}, \mathrm{Y}$ and $\mathrm{Z}$ positions, respectively. The SA volume for all the source nodes is about $14 \%$ bigger than the volume used in the PA, thus some extra dilution occurred in the SA model. The SA model placed the source at shallower cells within the uppermost aquifer. Most of the PA cells were within the tan clay confining layer.

Table 4. Aquifer source node locations

\begin{tabular}{cccccccc}
\hline $\begin{array}{c}\text { I } \\
\text { Index }\end{array}$ & $\begin{array}{c}\text { J } \\
\text { index }\end{array}$ & $\begin{array}{c}\text { SA } \\
\mathrm{K} \text { index }\end{array}$ & $\begin{array}{c}\text { PA } \\
\mathrm{K} \text { index }\end{array}$ & $\begin{array}{c}\text { SA } \\
\text { Volume } \\
\left(\mathrm{ft}^{3}\right)\end{array}$ & $\begin{array}{c}\text { PA } \\
\text { Volume } \\
\left(\mathrm{ft}^{3}\right)\end{array}$ & $\begin{array}{c}\text { SA Cell } \\
\text { Thickness } \\
(\mathrm{ft})\end{array}$ & $\begin{array}{c}\text { PA Cell } \\
\text { Thickness } \\
(\mathrm{ft})\end{array}$ \\
\hline 36 & 18 & 14 & 12 & & & 7.68 & 7.84 \\
37 & 18 & 13 & 12 & & & 8.01 & 7.48 \\
37 & 19 & 13 & 12 & & & 7.40 & 8.70 \\
38 & 19 & 13 & 13 & & & 7.45 & 7.45 \\
38 & 20 & 13 & 13 & & & 7.11 & 7.11 \\
39 & 20 & 14 & 12 & & & 7.05 & 6.11 \\
Totals & & & & $1,799,420$ & $1,581,000$ & 7.45 (avg.) & 7.45 (avg.) \\
\hline
\end{tabular}

\subsubsection{Reduced size of model}

The original aquifer model extended from multiple waste facilities to seeplines. The computer execution run times often were very long. Because the interest for PA purposes is the waste area and the 100-m well, the size of the modeling domain was reduced to encompass this smaller area plus a buffer. The buffer was added to ensure that the highest concentration was captured. Results from some test cases of the reduced size model showed results identical to those for the full-sized PA model.

However, in some cases the mass balance error for the reduced-size model were somewhat elevated. The boundary conditions were changed from a FLUX setting to a GRADIENT setting. The FLUX setting prevented all contamination from crossing the boundary (both from advection and diffusion). The GRADIENT setting only prevented diffusion from crossing the boundary while allowing contamination to be transported across the boundary by 
moving water (advection). Use of the GRADIENT boundary condition effectively eliminated the mass balance errors.

\subsubsection{High-Concentration I-129 waste}

As discussed in Section 3.1.1.6, high-concentration I-129 waste with waste-specific Kds were also analyzed for potential disposal as CIG.

\subsection{Groundwater Pathway Results}

The groundwater pathway results for wastes other than the $\mathrm{K}$ and $\mathrm{L}$ basin resins are shown in PA replacement tables and figures in Appendix A and Appendix B, respectively. In those tables, the radionuclides with a suffix of "_KB" refer to the $\mathrm{K}$ and $\mathrm{L}$ basin resin waste. In Appendix A, replacement Table 4.3-7 shows the contaminant fluxes to the water table, Table 5.1-7 shows the peak groundwater concentrations and Table 5.1-13 shows the calculated inventory limits for the groundwater pathway. Table 7.1-6 shows the minimum inventory limit for all the scenarios and pathways considered, namely the groundwater, air and radon pathways and the intruder scenarios.

\subsection{1 $\mathrm{K}$ and $\mathrm{L}$ basin wastes}

The effects of measuring waste-specific Kds for the $\mathrm{K}$ and $\mathrm{L}$ basin resin waste are shown in Table 5. The original PA assumed a waste similar to grout. The current SA used waste assumed to be in the presence of corrosion products from HICs or other containers, such as portable deionizers. Using the waste-specific Kds decreased the contaminant fluxes to the water table by about 3 orders of magnitude and decreased the peak well concentrations by about 2 orders of magnitude for both the Tc-99_KB and I-129_KB wastes. A much smaller improvement is seen for C-14_KB. Decreases in peak well concentrations directly translate into increases in inventory limits.

Table 5. Effect of measuring waste-specific Kds

\begin{tabular}{|c|c|c|c|}
\hline Nuclide & $\begin{array}{l}\text { Generic waste } \mathrm{Kd} \\
{[\text { Waste-specific } \mathrm{Kd}]}\end{array}$ & $\begin{array}{l}\text { Flux to WT (generic) } \\
\text { [Flux to WT (specific)] }\end{array}$ & $\begin{array}{l}\text { Well Conc. (generic) } \\
{[\text { Well Conc. (specific)] }}\end{array}$ \\
\hline C-14 & 2 & $4.92 \mathrm{E}-4$ & $1.77 \mathrm{E}+1$ \\
\hline [C-14_KB] & [140] & {$[4.22 \mathrm{E}-4]$} & {$[1.62 \mathrm{E}+1]$} \\
\hline Tc-99 & 0.36 & $1.32 \mathrm{E}-1$ & $1.20 \mathrm{E}+3$ \\
\hline [Tc-99_KB] & [810] & [5.37E-4] & {$[2.15 \mathrm{E}+1]$} \\
\hline $\mathrm{I}-129$ & 0.6 & $9.53 \mathrm{E}-2$ & $8.15 E+2$ \\
\hline [I-129_KB] & [3700] & [1.18E-4] & {$[4.71 \mathrm{E}+0]$} \\
\hline
\end{tabular}

\subsubsection{High-concentration I-129 waste}

The high-concentration I-129 wastes are presented in Table 6 arranged by order of Kd. Wastes that are not yet in the WITS have names in quotation marks. The "SIR 1200" waste is an anionic resin that replaced the Dowex $21 \mathrm{~K}$ resin in the F-Area Ground Water Treatment 
Unit (Kaplan, 2001). The "hypothetical waste" is hypothetical, but it provides another explicit modeling result and extends the range of the equation described below, in case a future waste stream has an extremely high $\mathrm{Kd}$ measured for it.

Table 6. Groundwater pathway results for high-concentration I-129 wastes

\begin{tabular}{|c|c|c|c|c|c|}
\hline Waste Description & $\begin{array}{l}\text { WITS } \\
\text { Name }\end{array}$ & $\begin{array}{c}\mathrm{Kd} \\
(\mathrm{ml} / \mathrm{g})\end{array}$ & $\begin{array}{l}\text { Peak Well Conc. } \\
\text { (pCi/L per } \mathrm{Ci})\end{array}$ & $\begin{array}{l}\text { Time } \\
\text { (years) }\end{array}$ & $\begin{array}{l}\text { Inv. Limit } \\
\text { (Ci) }\end{array}$ \\
\hline Generic & I-129 & 0.6 & $8.15 \mathrm{E}+02$ & $3.37 \mathrm{E}+02$ & $6.13 \mathrm{E}-04$ \\
\hline F-Area CG- 8 & I-129_G & 3 & $8.01 \mathrm{E}+02$ & $3.40 \mathrm{E}+02$ & $6.24 \mathrm{E}-04$ \\
\hline F-Area Filtercake & I-129_J & 12.4 & $6.75 \mathrm{E}+02$ & $3.66 \mathrm{E}+02$ & $7.41 \mathrm{E}-04$ \\
\hline H-Area CG-8 & I-129_H & 100 & $1.73 \mathrm{E}+02$ & $4.55 \mathrm{E}+02$ & 2.89E-03 \\
\hline H-Area Carbon & I-129_A & 320 & $5.45 \mathrm{E}+01$ & $6.62 \mathrm{E}+02$ & $9.17 \mathrm{E}-03$ \\
\hline ETF Activated Carbon & I-129_C & 600 & $2.91 \mathrm{E}+01$ & $9.27 \mathrm{E}+02$ & $1.72 \mathrm{E}-02$ \\
\hline H-Area Filtercake & I-129_F & 630 & 2.77E+01 & $9.55 \mathrm{E}+02$ & $1.81 \mathrm{E}-02$ \\
\hline F-Area Activated Carbon & I-129_B & 880 & $1.98 \mathrm{E}+01$ & $1.19 \mathrm{E}+03$ & $2.53 \mathrm{E}-02$ \\
\hline H-Area Dowex $21 \mathrm{~K}$ & I-129_E & 1980 & $8.81 \mathrm{E}+00$ & $2.23 \mathrm{E}+03$ & $5.68 \mathrm{E}-02$ \\
\hline F-Area Dowex $21 \mathrm{~K}$ & I-129_D & 2800 & $6.23 \mathrm{E}+00$ & $3.00 \mathrm{E}+03$ & 8.03E-02 \\
\hline ETF GT-73 & I-129_I & 3100 & $5.63 \mathrm{E}+00$ & $3.28 \mathrm{E}+03$ & 8.88E-02 \\
\hline K\&L Basin Waste & "I-129_KB" & 3700 & $4.71 \mathrm{E}+00$ & $3.85 \mathrm{E}+03$ & $1.06 \mathrm{E}-01$ \\
\hline SIR 1200 & "I-129_S" & 5762 & $3.03 \mathrm{E}+00$ & $5.80 \mathrm{E}+03$ & $1.65 \mathrm{E}-01$ \\
\hline Hypothetical & "I-129_10K" & 10000 & $1.74 \mathrm{E}+00$ & $9.77 \mathrm{E}+03$ & $2.87 \mathrm{E}-01$ \\
\hline
\end{tabular}

Inventory limits versus Kds are plotted in Figure 2. The top plot shows all the data. It is apparent that a linear relationship does not exist for all the data. However, a bilinear relationship appears to be a reasonable approximation with an intersection at the third data point (F-Area Filtercake) with a $\mathrm{Kd}$ of $12.4 \mathrm{ml} / \mathrm{g}$ and an inventory limit of $7.41 \mathrm{E}-4 \mathrm{Ci}$. The lower two plots show the data points used to generate the equations below. The best-fit equations relating the inventory limit to the $\mathrm{Kd}$ are

\section{Equation 1}

InventoryLimit $(\mathrm{Ci})=1.12 E-05 *\left[K_{d}(\mathrm{ml} / \mathrm{g})\right]+6.00 E-4 \quad K_{d} \leq 12.4 \mathrm{ml} / \mathrm{g}$

\section{Equation 2}

InventoryLimit $(\mathrm{Ci})=2.87 \mathrm{E}-05 *\left[K_{d}(\mathrm{ml} / \mathrm{g})\right]-1.76 E-5 \quad 12.4 \mathrm{ml} / \mathrm{g}<K_{d} \leq 10000 \mathrm{ml} / \mathrm{g}$

The Kds for the high-concentration wastes were back-fitted into the two equations. The resulting back-fitted inventory limits agreed extremely well with the model results. The back-fitted inventory limits were lower than the model results for the two smallest Kds used to develop Equation 2, but those results are conservative. All other back-fitted inventory limits were not appreciably different than the model results. Thus, for future wastes with waste-specific Kds that have not previously been modeled, it is recommended that the two equations be used as is, without any other corrections. Alternatively, linear interpolation between pairs of results is acceptable. 


\subsubsection{ETF activated carbon vessels}

One option for disposal of three ETF activated carbon vessels is to dispose of them as CIG (Collard, 2002). The two nuclides of concern were identified as H-3 and I-129. The limits for those nuclides are $8.62 \mathrm{E} 7 \mathrm{Ci}$ and $1.72 \mathrm{E}-2 \mathrm{Ci}$, respectively. These limits are compared to the inventories in Section 5.3.
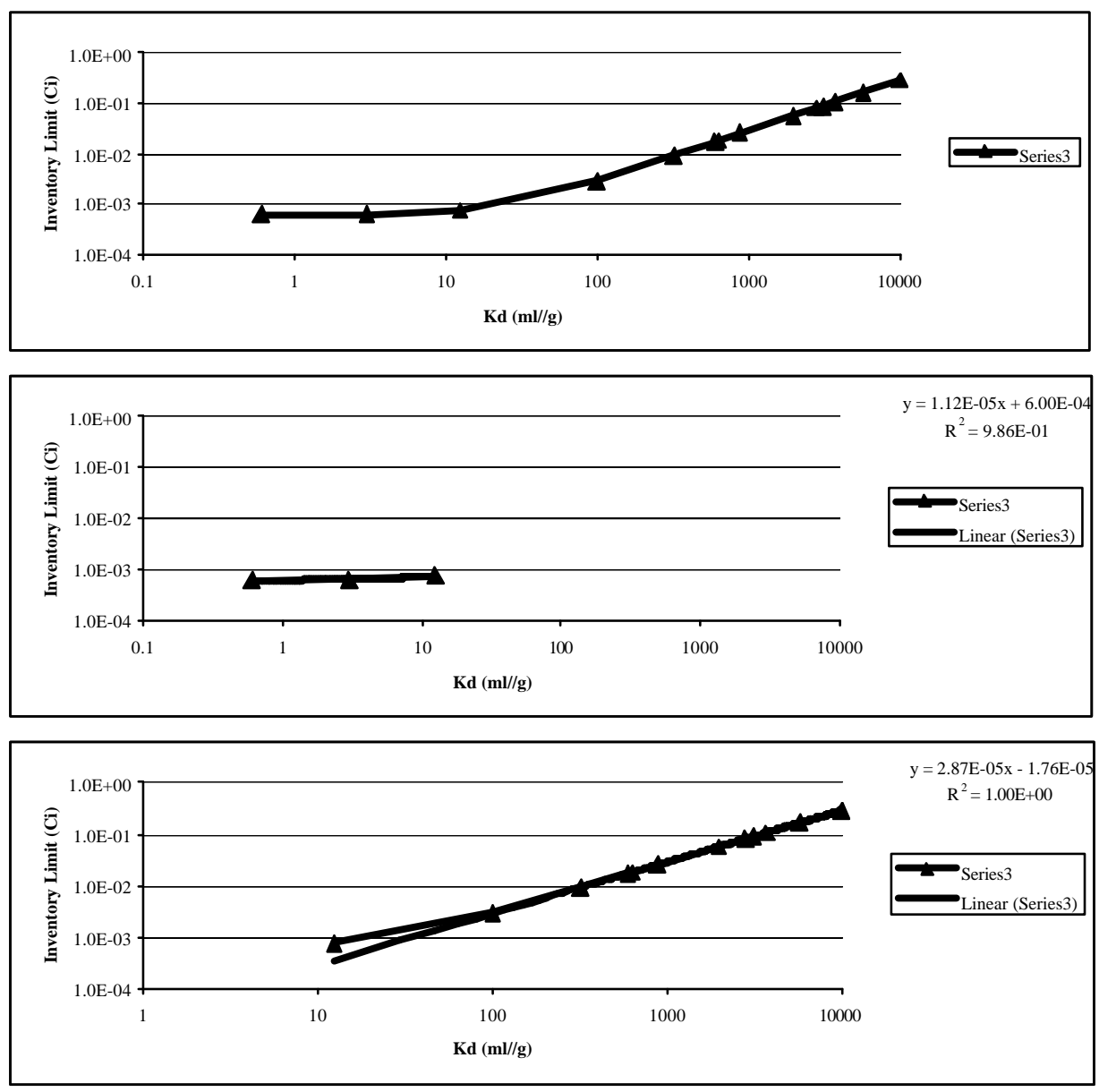

Figure 2. Inventory limits versus Kd for high-concentration I-129 waste as CIG

\subsection{INTRUDER AND AIR PATHWAY ANALYSIS}

\subsection{Intruder Analysis}

The intruder analysis for the CSEW disposal unit was revised to reflect the changes in the groundwater modeling, the addition of a number of radionuclides and incorporation of the wastes from the $\mathrm{K}$ and $\mathrm{L}$ basins. These results are presented in the following replacement tables: 
PA Replacement Table

6.3-10

6.3-16

6.3-20

7.1-6
Intruder Analysis

Agriculture at 700 years

Resident at 100 years

Post-Drilling at 300 years

Inventory Limits (when most restrictive)

The disposal limits for each radionuclide and the most limiting pathway are shown in Table 7.1-6 in Appendix A. I-129_XX in the tables refers to wastes with waste-specific Kds for I129 , other than the $\mathrm{K}$ and $\mathrm{L}$ basin waste.

\subsection{Air Pathway}

Previous analyses of the air pathway were done representing the disposal unit as a point source. This introduced a great deal of conservatism for the case where the hypothetical receptor is at the facility boundary, 100 meters away. Calculations were done by the SRTC Environmental Analysis group to give the dose per unit release for $\mathrm{H}-3$ and $\mathrm{C}-14$ for release from a set of five trenches to a receptor 100 meters away and at the site boundary (Appendix C). The results are shown in Appendix C, Table 1.

Subsequent analyses (Simpkins, 2003) for multiple sets of trenches showed that the relative concentrations at the $100 \mathrm{~m}$ boundary would increase by about $30 \%$ for 2 sets of trenches, by about $50 \%$ for 3 sets of trenches and by about $60 \%$ for 4 sets of trenches. Thus the incremental increase in concentration at the receptor for each new set of trenches is $30 \%$, $20 \%$ and $10 \%$. As the incremental increases in concentration continue to diminish, the $13^{\text {th }}$ trench should provide an incremental increase of only $0.01 \%$ (assuming the incremental increase for each trench is $1 / 2$ that of the previous trench). At that rate, the $5^{\text {th }}$ through $13^{\text {th }}$ trenches will only increase the concentration by about $12 \%$. For all thirteen trenches, the concentration at the receptor would be about $72 \%$ higher than for one trench. To accommodate the effects of multiple trenches, the concentrations at the $100 \mathrm{~m}$ boundary from one trench will be increased by $100 \%$, thus the inventory limits for the air pathway will be halved.

For the receptor located at the SRS site boundary a point source was assumed for one set of trenches. To consider the net effect of all $21 \mathrm{LAW}$ vault footprints that are identified on drawings, the concentration for one set of trenches will be multiplied by one-half of 21 , because the footprints are not all aligned.

The dose factors in Table 4.2-2 (Appendix A), the DOE performance objective of 10 mrem/year for the atmospheric pathway, and decay over the 100 year institutional control period for the 100 meter exposure point were used to calculate inventory limits for $\mathrm{H}-3$ and C-14 for a set of five CSEW trenches. These results are shown in Appendix A, Table 5.2-1.

The disposal limits for each radionuclide and the most limiting pathway are shown in Table 7.1-6 in Appendix A. 


\subsection{CONCLUSIONS}

\subsection{Comparison of Old and Revised Inventory Limits}

Table 7 shows a comparison of the old limits and revised inventory limits. Nuclides that had a change in the limiting pathway included $\mathrm{C}-14, \mathrm{Sr}-90$ and several $\mathrm{U}$ and Pu isotopes. The C-14 pathway changed to groundwater because a new air analysis was completed. The Sr-90 pathway changed to groundwater because of changes in the groundwater model, but the limit was reduced by less than a factor of two. The limiting pathway changed to groundwater for several $\mathrm{U}$ and $\mathrm{Pu}$ isotopes because the solubility-limit was not invoked in the groundwater model. 
Table 7. Comparison of old and revised inventory limits

\begin{tabular}{|c|c|c|c|c|c|}
\hline Radionuclide & $\begin{array}{c}\text { New Limit } \\
(\mathrm{Ci})\end{array}$ & $\begin{array}{l}\text { Limiting } \\
\text { Pathway }\end{array}$ & Radionuclide & $\begin{array}{l}\text { Old Limit } \\
(\mathrm{Ci})\end{array}$ & $\begin{array}{l}\text { Limiting } \\
\text { Pathway }\end{array}$ \\
\hline$\overline{\mathrm{H}-3}$ & $4.1 \mathrm{E}+05$ & air & H-3 & $3.20 \mathrm{E}+05$ & air \\
\hline C- 14 & $5.7 \mathrm{E}+01$ & gw & C-14 & $2.70 \mathrm{E}+00$ & air \\
\hline C-14_KB & $6.2 \mathrm{E}+01$ & gw & & & \\
\hline Co-60 & $2.1 \mathrm{E}+09$ & resident & Сo-60 & $2.10 \mathrm{E}+09$ & resident \\
\hline $\mathrm{Ni}-59$ & $9.3 \mathrm{E}+02$ & gw & $\mathrm{Ni}-59$ & $2.50 \mathrm{E}+03$ & gw \\
\hline $\mathrm{Ni}-63$ & $1.3 \mathrm{E}+06$ & post-drilling & $\mathrm{Ni}-63$ & $1.30 \mathrm{E}+06$ & post-drilling \\
\hline Se-79 & $9.3 \mathrm{E}+01$ & gw & Se-79 & $8.10 \mathrm{E}+01$ & gw \\
\hline $\mathrm{Rb}-87$ & $1.4 \mathrm{E}+01$ & gw & & & \\
\hline Sr-90+d & $1.6 \mathrm{E}+05$ & gw & Sr- $90+d$ & $2.30 \mathrm{E}+05$ & post-drilling \\
\hline $\mathrm{Zr}-93+\mathrm{d}$ & $4.0 \mathrm{E}+04$ & gw & $\mathrm{Zr}-93+\mathrm{d}$ & $1.50 \mathrm{E}+01$ & gw \\
\hline $\mathrm{Nb}-94$ & $2.3 \mathrm{E}+00$ & agriculture & & & \\
\hline Мo-93 & $6.3 \mathrm{E}+06$ & agriculture & & & \\
\hline Tc-99 & $3.8 \mathrm{E}-01$ & gw & Tc-99 & $3.50 \mathrm{E}-01$ & gw \\
\hline Tc-99_KB & $2.1 \mathrm{E}+01$ & gw & & & \\
\hline Pd-107 & $1.8 \mathrm{E}+03$ & gw & Pd-107 & $1.80 \mathrm{E}+01$ & gw \\
\hline Cd-113m & $2.4 \mathrm{E}+04$ & agriculture & & & \\
\hline Sn-121m & $1.2 \mathrm{E}+06$ & agriculture & & & \\
\hline Sn-126+d & $4.6 \mathrm{E}+00$ & agriculture & Sn-126+d & $5.20 \mathrm{E}+00$ & agriculture \\
\hline I- 129 & $6.1 \mathrm{E}-04$ & gw & I- 129 & $4.20 \mathrm{E}-04$ & gw \\
\hline I- $129 \mathrm{~KB}$ & $1.1 \mathrm{E}-01$ & $\mathrm{gw}$ & & & \\
\hline I-129_A & $9.2 \mathrm{E}-03$ & gw & & & \\
\hline I-129_B & $2.5 \mathrm{E}-02$ & gw & & & \\
\hline I-129_C & $1.7 \mathrm{E}-02$ & gw & & & \\
\hline I-129_D & 8.0E-02 & gw & & & \\
\hline I-129_E & $5.7 \mathrm{E}-02$ & gw & & & \\
\hline I-129_F & $1.8 \mathrm{E}-02$ & gw & & & \\
\hline I-129_G & $6.2 \mathrm{E}-04$ & gw & & & \\
\hline I-129_H & 2.9E-03 & gw & & & \\
\hline I-129_I & 8.9E-02 & gw & & & \\
\hline I-129_J & 7.4E-04 & gw & & & \\
\hline I-129_S & $1.7 \mathrm{E}-01$ & $\mathrm{gw}$ & & & \\
\hline I-129_10K & 2.9E-01 & gw & & & \\
\hline Cs -135 & $5.1 \mathrm{E}+02$ & gw & Cs -135 & $2.30 \mathrm{E}+01$ & gw \\
\hline Cs $-137+d$ & $2.2 \mathrm{E}+06$ & resident & Cs $-137+d$ & $2.20 \mathrm{E}+06$ & resident \\
\hline Sm- 151 & $3.1 \mathrm{E}+07$ & post-drilling & Sm-151 & $3.10 \mathrm{E}+07$ & post-drilling \\
\hline $\mathrm{Eu}-152$ & $2.4 \mathrm{E}+06$ & resident & & & \\
\hline Eu-154 & $3.6 \mathrm{E}+07$ & resident & $\mathrm{Eu}-154$ & $3.60 \mathrm{E}+07$ & resident \\
\hline Th-228 & $4.6 \mathrm{E}+02$ & agriculture & & & \\
\hline Th-232+d & $1.4 \mathrm{E}+00$ & agriculture & & & \\
\hline $\mathrm{U}-232+\mathrm{d}$ & $1.7 \mathrm{E}+03$ & agriculture & $\mathrm{U}-232+\mathrm{d}$ & $1.70 \mathrm{E}+03$ & agriculture \\
\hline $\mathrm{U}-233+\mathrm{d}$ & $2.3 \mathrm{E}+01$ & gw & $\mathrm{U}-233+\mathrm{d}$ & $4.10 \mathrm{E}+01$ & agriculture \\
\hline $\mathrm{U}-234+\mathrm{d}$ & $2.3 \mathrm{E}+01$ & gw & $\mathrm{U}-234+\mathrm{d}$ & $4.90 \mathrm{E}+01$ & radon \\
\hline $\mathrm{U}-235+\mathrm{d}$ & $1.1 \mathrm{E}+01$ & gw & $\mathrm{U}-235+\mathrm{d}$ & $2.30 \mathrm{E}+01$ & agriculture \\
\hline U-236 & $2.4 \mathrm{E}+01$ & gw & U-236 & $4.60 \mathrm{E}+02$ & agriculture \\
\hline $\mathrm{U}-238+\mathrm{d}$ & $1.2 \mathrm{E}+02$ & agriculture & $\mathrm{U}-238+\mathrm{d}$ & $1.20 \mathrm{E}+02$ & agriculture \\
\hline Np-237 & $3.7 \mathrm{E}-01$ & gw & & & \\
\hline $\mathrm{Pu}-238+\mathrm{d}$ & $1.4 \mathrm{E}+04$ & post-drilling & $\mathrm{Pu}-238+\mathrm{d}$ & $1.40 \mathrm{E}+04$ & post-drilling \\
\hline $\mathrm{Pu}-239+\mathrm{d}$ & $1.3 \mathrm{E}+02$ & agriculture & $\mathrm{Pu}-239+\mathrm{d}$ & $1.30 \mathrm{E}+02$ & agriculture \\
\hline
\end{tabular}


Table 7. Comparison of old and revised inventory limits

\begin{tabular}{lccccc}
\hline Radionuclide & $\begin{array}{c}\text { New Limit } \\
(\mathrm{Ci})\end{array}$ & $\begin{array}{c}\text { Limiting } \\
\text { Pathway }\end{array}$ & Radionuclide & $\begin{array}{c}\text { Old Limit } \\
(\mathrm{Ci})\end{array}$ & $\begin{array}{c}\text { Limiting } \\
\text { Pathway }\end{array}$ \\
\hline $\mathrm{Pu}-240+\mathrm{d}$ & $2.7 \mathrm{E}+00$ & $\mathrm{gw}$ & $\mathrm{Pu}-240+\mathrm{d}$ & $1.30 \mathrm{E}+02$ & agriculture \\
$\mathrm{Pu}-241+\mathrm{d}$ & $8.0 \mathrm{E}+03$ & agriculture & $\mathrm{Pu}-241+\mathrm{d}$ & $8.00 \mathrm{E}+03$ & agriculture \\
$\mathrm{Pu}-242+\mathrm{d}$ & $1.0 \mathrm{E}+00$ & $\mathrm{gw}$ & $\mathrm{Pu}-242+\mathrm{d}$ & $1.30 \mathrm{E}+02$ & agriculture \\
$\mathrm{Pu}-244+\mathrm{d}$ & $1.1 \mathrm{E}+00$ & $\mathrm{gw}$ & & & \\
$\mathrm{Am}-241+\mathrm{d}$ & $2.7 \mathrm{E}+02$ & agriculture & Am-241+d & $2.70 \mathrm{E}+02$ & agriculture \\
$\mathrm{Am}-242 \mathrm{~m}+\mathrm{d}$ & $2.1 \mathrm{E}+03$ & agriculture & & & \\
$\mathrm{Am}-243+\mathrm{d}$ & $2.1 \mathrm{E}+01$ & agriculture & & & \\
$\mathrm{Cm}-242+\mathrm{d}$ & $2.8 \mathrm{E}+06$ & post-drilling & & & \\
$\mathrm{Cm}-243$ & $2.5 \mathrm{E}+06$ & post-drilling & & & \\
$\mathrm{Cm}-244+\mathrm{d}$ & $4.3 \mathrm{E}+04$ & agriculture & & & \\
$\mathrm{Cm}-245+\mathrm{d}$ & $3.0 \mathrm{E}+01$ & agriculture & & & \\
$\mathrm{Cm}-246$ & $1.2 \mathrm{E}+02$ & agriculture & & & \\
$\mathrm{Cm}-247+\mathrm{d}$ & $9.5 \mathrm{E}+00$ & agriculture & & \\
$\mathrm{Cm}-248+\mathrm{d}$ & $3.1 \mathrm{E}+01$ & agriculture & & \\
$\mathrm{Bk}-249+\mathrm{d}$ & $1.5 \mathrm{E}+04$ & agriculture & & \\
$\mathrm{Cf}-249+\mathrm{d}$ & $3.6 \mathrm{E}+01$ & agriculture & & \\
$\mathrm{Cf}-250+\mathrm{d}$ & $8.2 \mathrm{E}+05$ & agriculture & & \\
$\mathrm{Cf}-251$ & $1.4 \mathrm{E}+03$ & agriculture & & \\
$\mathrm{Cf}-252+\mathrm{d}$ & $4.5 \mathrm{E}+07$ & post-drilling & & & \\
\hline
\end{tabular}

Nuclides where limits increased or decreased by more than a factor of two included H-3, C-14, Ni-59, Zr-93, Pd-107, Cs-135 and several U and Pu isotopes. The H-3 and C-14 limits changed because a new air analysis was completed. The Ni59, Zr-93, Pd-107, and Cs-135 limits changed because of changes in the groundwater model. The $\mathrm{U}$ and $\mathrm{Pu}$ isotope limits changed because the solubility-limit was not invoked in the groundwater model.

\subsection{Inventory and Volume Consumption for $K$ and $L$ Basin Resins}

The current and projected inventory for $\mathrm{K}$ and $\mathrm{L}$ basin resins is shown in Table 8 . Projections for C-14_KB and Np-237 and to a lesser extent I-129_KB appear to consume large portions of their inventory limits. However, the consumption is shown for only 1 set of 5 CIG trenches. The projections are through the year 2035, thus as more CIG trenches are excavated and filled, the $\mathrm{K}$ and L basin waste is likely to be spread over more than $5 \mathrm{CIG}$ trenches.

Spreading the K and L basin waste over more trenches will decrease the percentage consumption figures shown in Table 8 . There is no current estimate available for the number of CIG trenches that will be needed by 2035 , because buildings and equipment that ultimately will be disposed as CIG have not yet been identified. 
Table 8. Current and projected inventory for $K$ and $L$ basin compared to limits

\begin{tabular}{|c|c|c|c|c|c|}
\hline Isotope & $\begin{array}{l}\text { New CIG } \\
\text { SA Limit } \\
\quad(\text { Ci) }\end{array}$ & $\begin{array}{l}\text { Current } K \text { and } L \\
\text { Inventory } \\
\text { (Ci) }\end{array}$ & $\begin{array}{c}\text { Fraction of } \\
\text { SA Limit }\end{array}$ & $\begin{array}{l}\text { Projected } \mathrm{K} \text { and } \mathrm{L} \\
\text { Inventory } \\
\text { (Ci) }\end{array}$ & $\begin{array}{c}\text { Fraction of } \\
\text { SA Limit }\end{array}$ \\
\hline$\overline{\mathrm{H} 3}$ & $4.1 \mathrm{E}+05$ & $8.54 \mathrm{E}-01$ & $2.08 \mathrm{E}-06$ & $1.52 \mathrm{E}+01$ & $3.71 \mathrm{E}-05$ \\
\hline C14_KB & $6.2 \mathrm{E}+01$ & $8.66 \mathrm{E}-01$ & $1.40 \mathrm{E}-02$ & $1.57 \mathrm{E}+02$ & 2.53E+00 \\
\hline $\mathrm{CO} 60$ & $2.1 \mathrm{E}+09$ & $3.79 \mathrm{E}-03$ & $1.80 \mathrm{E}-12$ & $0.00 \mathrm{E}+00$ & $0.00 \mathrm{E}+00$ \\
\hline SR90 & $1.6 \mathrm{E}+05$ & $1.24 \mathrm{E}+01$ & $7.75 \mathrm{E}-05$ & $2.27 \mathrm{E}+03$ & $1.42 \mathrm{E}-02$ \\
\hline Y90 & No Limit & $1.24 \mathrm{E}+01$ & $0.00 \mathrm{E}+00$ & $2.27 \mathrm{E}+03$ & $0.00 \mathrm{E}+00$ \\
\hline TC99_KB & $2.1 \mathrm{E}+01$ & $1.79 \mathrm{E}-02$ & 8.52E-04 & $2.81 \mathrm{E}+00$ & $1.34 \mathrm{E}-01$ \\
\hline RU106 & No Limit & $3.82 \mathrm{E}-04$ & $0.00 \mathrm{E}+00$ & $0.00 \mathrm{E}+00$ & $0.00 \mathrm{E}+00$ \\
\hline RH106 & No Limit & $3.82 \mathrm{E}-04$ & $0.00 \mathrm{E}+00$ & $0.00 \mathrm{E}+00$ & $0.00 \mathrm{E}+00$ \\
\hline SB125 & No Limit & $4.36 \mathrm{E}-04$ & $0.00 \mathrm{E}+00$ & $0.00 \mathrm{E}+00$ & $0.00 \mathrm{E}+00$ \\
\hline I129_KB & $1.1 \mathrm{E}-01$ & $1.33 \mathrm{E}-04$ & $1.21 \mathrm{E}-03$ & $1.78 \mathrm{E}-02$ & $1.62 \mathrm{E}-01$ \\
\hline CS137 & $2.2 \mathrm{E}+06$ & $1.93 \mathrm{E}+01$ & $8.77 \mathrm{E}-06$ & $3.52 \mathrm{E}+03$ & $1.60 \mathrm{E}-03$ \\
\hline BA137M & No Limit & $1.83 \mathrm{E}+01$ & $0.00 \mathrm{E}+00$ & $3.33 E+03$ & $0.00 \mathrm{E}+00$ \\
\hline $\mathrm{U} 234$ & $2.3 \mathrm{E}+01$ & $2.41 \mathrm{E}-03$ & $1.05 \mathrm{E}-04$ & $4.15 \mathrm{E}-01$ & $1.80 \mathrm{E}-02$ \\
\hline $\mathrm{U} 235$ & $1.1 \mathrm{E}+01$ & $4.88 \mathrm{E}-05$ & $4.44 \mathrm{E}-06$ & 8.99E-03 & $8.17 \mathrm{E}-04$ \\
\hline $\mathrm{U} 238$ & $1.2 \mathrm{E}+02$ & $1.86 \mathrm{E}-03$ & $1.55 \mathrm{E}-05$ & $3.43 \mathrm{E}-01$ & $2.86 \mathrm{E}-03$ \\
\hline NP237 & $3.7 \mathrm{E}-01$ & $5.25 \mathrm{E}-03$ & $1.42 \mathrm{E}-02$ & $9.68 \mathrm{E}-01$ & $2.62 \mathrm{E}+00$ \\
\hline PU238 & $1.4 \mathrm{E}+04$ & $2.14 \mathrm{E}-02$ & $1.53 \mathrm{E}-06$ & 9.87E-01 & 7.05E-05 \\
\hline PU239 & $1.3 \mathrm{E}+02$ & 4.87E-02 & $3.75 \mathrm{E}-04$ & $2.77 \mathrm{E}+00$ & $2.13 \mathrm{E}-02$ \\
\hline PU241 & $8.0 \mathrm{E}+03$ & $1.29 \mathrm{E}-01$ & $1.61 \mathrm{E}-05$ & $6.18 \mathrm{E}+00$ & $7.73 \mathrm{E}-04$ \\
\hline AM241 & $2.7 \mathrm{E}+02$ & $3.15 \mathrm{E}-02$ & $1.17 \mathrm{E}-04$ & $5.64 \mathrm{E}+00$ & $2.09 \mathrm{E}-02$ \\
\hline Sum & & & $3.10 \mathrm{E}-02$ & & $5.53 \mathrm{E}+00$ \\
\hline \multicolumn{2}{|c|}{ Volume Consumed } & & $0.50 \%$ & & $6.50 \%$ \\
\hline
\end{tabular}

\subsection{ETF Activated Carbon Inventory and Volume Consumption}

Table 9 contains inventories from Collard, 2002, inventory limits from this SA and the percent of the inventory consumption for H-3 and I-129 (I-129_C) in three ETF activated carbon vessels awaiting disposal. The percent of the facility volume $\left(1.02 \mathrm{E}+06 \mathrm{ft}^{3}\right.$ or $\left.2.88 \mathrm{E}+04 \mathrm{~m}^{3}\right)$ consumed by the vessels $\left(1000 \mathrm{ft}^{3}\right.$ each) is shown for comparison. The $\mathrm{H}-3$ poses no problems because it would consume about $2 \mathrm{E}-7$ percent of the inventory limit. However, the I-129 would consume about 69 percent of the inventory limit, while occupying less than 1 percent of the volume.

Table 9. ETF activated carbon vessel inventory and facility volume consumption

\begin{tabular}{lccccccc}
\hline \multicolumn{3}{c}{ Hes Inventory Values } & \multicolumn{2}{c}{ I-129 Inventory Values } \\
& $\begin{array}{c}\text { Inventory } \\
(\mathrm{Ci})\end{array}$ & $\begin{array}{c}\text { Limit } \\
(\mathrm{Ci})\end{array}$ & $\begin{array}{c}\text { Consumed } \\
(\%)\end{array}$ & $\begin{array}{c}\text { Inventory } \\
(\mathrm{Ci})\end{array}$ & $\begin{array}{c}\text { Limit } \\
(\mathrm{Ci})\end{array}$ & $\begin{array}{c}\text { Consumed } \\
(\%)\end{array}$ & $\begin{array}{c}\text { Facility Vol. } \\
\text { Consumed }(\%)\end{array}$ \\
\hline$\# 16$ & $7.56 \mathrm{E}-2$ & & & $3.90 \mathrm{E}-3$ & & & \\
$\# 5$ & $2.12 \mathrm{E}-2$ & & & $1.35 \mathrm{E}-3$ & & & \\
$\# 9$ & $3.68 \mathrm{E}-2$ & & & $6.56 \mathrm{E}-3$ & & & \\
Total & $1.34 \mathrm{E}-1$ & $4.1 \mathrm{E}+05$ & $3.27 \mathrm{E}-05$ & $1.18 \mathrm{E}-2$ & $1.7 \mathrm{E}-02$ & $6.9 \mathrm{E}+01$ & 0.30 \\
\hline
\end{tabular}




\subsection{REFERENCES}

\subsection{Groundwater Pathway References}

Collard, L.B. 2002. Evaluation of Proposed New LLW Disposal Activity: Disposal of ETF Activated Carbon Vessels in Trenches, WSRC-TR-2002-00339, Revision 0, Westinghouse Savannah River Company, Aiken, South Carolina, 29808, September 3.

Collard, L.B. 2003. Evaluation of Porposed New LLW Disposal Activity; Disposal of Waste Resins Generated from the K and L Disassembly Basin Facilities, WSRC-TR-2002-00466, Revision 0, Westinghouse Savannah River Company, Aiken, South Carolina, 29808, October 15.

Cook, J.R. 2002. Special Analysis: Correction and Update of E-Area Disposal Limits, WSRC-TR-2002-00047, Revision 2, Westinghouse Savannah River Company, Aiken, South Carolina, 29808, May 2.

Cook, J.R. 2003. Variations in Trench Dimensions for Components in Grout Disposals, WSRC-RP-2003-00394, Revision 0, Westinghouse Savannah River Company, Aiken, South Carolina, 29808, April 15.

Cook, J.R., McDowell-Boyer, L., A.D. Yu, D.C. Kocher, E.L. Wilhite, H. Holmes-Burns, and K.E. Young. 1999. Spcial Analysis for Disposal of Cement-Stabilized Encapsulated Waste at the EArea Low-Level Waste Facility, WSRC-RP-99-00596, Revision 0, Westinghouse Savannah River Company, Aiken, South Carolina, 29808, December 8.

Flach, G.P. and L.B. Collard 2003. An Evaluation of Aquifer Source Node Location Alternatives for E-Area Slit Trench Performance Assessment, WSRC-TR-2003-00123, Revision 0, Westinghouse Savannah River Company, Aiken, South Carolina, 29808, March 28.

Kaplan, D.I.. 2001. ${ }^{129} I-K_{d}$ Values of SIR-1200 Resins Used in the F-Area Ground Water Treatment Unit, WSRC-TR-2001-00346, Revision 0, Westinghouse Savannah River Company, Aiken, South Carolina, 29808, July 30.

Kaplan, D.I.. and C. Coffee 2002. Distribution Coefficients (Kd Values) for Waste Resins Generated from the $K \&$ L Disassembly Basin Facilities, WSRC-TR-2002-00349, Revision 0, Westinghouse Savannah River Company, Aiken, South Carolina, 29808, August 9.

McDowell-Boyer, L., A.D. Yu, J.R. Cook, D.C. Kocher, E.L. Wilhite, H. Holmes-Burns, and K.E. Young. 2000. Radiological Performance Assessment for the E-Area Low Level Waste Facility, WSRC-RP-94-218, Revision 1, Westinghouse Savannah River Company, Aiken, South Carolina, 29808, January 31.

Phifer, M.A.. and L.B. Collard 2003. Unreviewed Disposal Question Evaluation: Backfill Soil Compaction Requirements, WSRC-TR-2003-00081, Revision 0, Westinghouse Savannah River Company, Aiken, South Carolina, 29808, February 6. 


\subsection{Intruder and Air Pathway References}

Beyer,W.H. 1981, CRC Standard Math Tables, 26th edition, CRC Press Inc. Boca Raton, FL.

Beres, D.A. 1990, The Clean Air Act Assessment Package-1988 (CAP-88) A Dose and Risk Assessment Methodology for Radionuclide Emissions to Air, U.S. Environmental Protection Agency Contract No. 68-D9-0170, Washington, D.C.

Moore, R.E., C.F. Baes III, L.M. McDowell-Boyer, A.P. Watson, F.O. Hoffman, J.C. Pleasant, C.W. Miller 1979, AIRDOS-EPA: A Computerized Methodology for Estimating Environmental Concentrations and Dose to Man from Airborne Releases of Radionuclides, Oak Ridge National Laboratory Report: ORNL-5532, Oak Ridge, TN.

Napier, B.A. 2002, GENII Version 2 Software Design Document, Prepared for U.S. Environmental Protection Agency under Contract DE-AC06-76RLO 1830, Washington, D.C.

Simpkins, A.A. 1998, Modeling Of Releases from the New Burial Ground, E Area-Additional Radionuclides, Westinghouse Savannah River Co Inter-Office Memorandum: SRT-EST-98-341, Westinghouse Savannah River Company, Aiken, SC.

Simpkins, A.A. 1999, MAXDOSE-SR: A Routine-Release Atmospheric Dose Model Used at SRS, Westinghouse Savannah River Company Report: WSRC-TR-99-00281, Westinghouse Savannah River Company, Aiken, SC.

Simpkins, A.A. 2003, Personal Communication.

U.S. DOE 1997, Hazard Categorization and Accident Analysis Techniques for Compliance with DOE Order 5480.23 Nuclear Safety Analysis Reports. U.S. Department of Energy Standard: DOESTD-1027-1992, (Change Notice No. 1, September 1997), Washington, D.C.

U.S. EPA 2002, National Emission Standards for Hazardous Air Pollutants; Radionuclides, U.S. Environmental Protection Agency. Title 40 Code of Federal Regulations, Part 61, Washington, D.C.

U.S. NRC 1977, Methods for Estimating Atmospheric Transport and Dispersion of Gaseous Effluents in Routine Releases from Light-Water-Cooled Reactors, U.S. Nuclear Regulatory Commission Regulatory Guide 1.111, Washington, D.C. 
Appendix A. PA Replacement Tables

Table 4.1-2 Parameters Used in Screening of Radionuclide Inventories for E-Area LowLevel Waste Facility Disposal Units and Lists of Radionuclides for Further Analysis with Respect to Off-Site Public Exposures

\begin{tabular}{|c|c|c|c|}
\hline $\begin{array}{l}\text { Type of } \\
\text { Disposal Unit }\end{array}$ & $\begin{array}{l}\text { Depth of } \\
\text { Waste in } \\
\text { Disposal } \\
\text { Unit (m) }\end{array}$ & $\begin{array}{l}\text { Area of } \\
\text { Waste in } \\
\text { Disposal } \\
\text { Unit }\left(\mathrm{m}^{2}\right)\end{array}$ & $\begin{array}{c}\text { Radionuclides Identified as Potentially } \\
\text { Significant Contributors to Dose Related to } \\
\text { Water Resource Impacts Analysis }\end{array}$ \\
\hline LAW Vaults & 3 & 8624 & $\begin{array}{l}{ }^{3} \mathrm{H},{ }^{14} \mathrm{C},{ }^{59} \mathrm{Ni},{ }^{79} \mathrm{Se},{ }^{87} \mathrm{Rb},{ }^{90} \mathrm{Sr}+\mathrm{d},{ }^{93} \mathrm{Zr}+\mathrm{d},{ }^{94} \mathrm{Nb}, \\
{ }^{99} \mathrm{Tc},{ }^{107} \mathrm{Pd},{ }^{126} \mathrm{Sn}+\mathrm{d},{ }^{129} \mathrm{I},{ }^{135} \mathrm{Cs},{ }^{232} \mathrm{Th}+\mathrm{d}, \\
{ }^{232} \mathrm{U}+\mathrm{d},{ }^{23} \mathrm{U}+\mathrm{d},{ }^{234} \mathrm{U}+\mathrm{d},{ }^{235} \mathrm{U}+\mathrm{d},{ }^{236} \mathrm{U},{ }^{237} \mathrm{~Np}, \\
{ }^{238} \mathrm{U}+\mathrm{d},{ }^{238} \mathrm{Pu}+\mathrm{d},{ }^{239} \mathrm{Pu}+\mathrm{d},{ }^{240} \mathrm{Pu}+\mathrm{d},{ }^{241} \mathrm{Pu}+\mathrm{d}, \\
{ }^{242} \mathrm{Pu}+\mathrm{d},{ }^{244} \mathrm{Pu},{ }^{241} \mathrm{Am}+\mathrm{d},{ }^{243} \mathrm{Am}+\mathrm{d},{ }^{244} \mathrm{Cm}+\mathrm{d}, \\
{ }^{245} \mathrm{Cm}+\mathrm{d},{ }^{246} \mathrm{Cm},{ }^{247} \mathrm{Cm}+\mathrm{d},{ }^{248} \mathrm{Cm}+\mathrm{d},{ }^{249} \mathrm{Cf}+\mathrm{d}, \\
{ }^{252} \mathrm{Cf}+\mathrm{d}\end{array}$ \\
\hline IL Vaults & 6 & 728 & $\begin{array}{l}{ }^{3} \mathrm{H},{ }^{14} \mathrm{C},{ }^{59} \mathrm{Ni},{ }^{79} \mathrm{Se},{ }^{90} \mathrm{Sr}+\mathrm{d},{ }^{93} \mathrm{Zr}+\mathrm{d},{ }^{99} \mathrm{Tc},{ }^{107} \mathrm{Pd}, \\
{ }^{126} \mathrm{Sn}+\mathrm{d},{ }^{129} \mathrm{I},{ }^{135} \mathrm{Cs},{ }^{232} \mathrm{Th}+\mathrm{d},{ }^{232} \mathrm{U}+\mathrm{d},{ }^{233} \mathrm{U}+\mathrm{d}, \\
{ }^{234} \mathrm{U}+\mathrm{d},{ }^{235} \mathrm{U}+\mathrm{d},{ }^{236} \mathrm{U},{ }^{237} \mathrm{~Np},{ }^{238} \mathrm{U}+\mathrm{d},{ }^{238} \mathrm{Pu}+\mathrm{d}, \\
{ }^{239} \mathrm{Pu}+\mathrm{d},{ }^{240} \mathrm{Pu}+\mathrm{d},{ }^{241} \mathrm{Pu}+\mathrm{d},{ }^{242} \mathrm{Pu}+\mathrm{d},{ }^{244} \mathrm{Pu}, \\
{ }^{241} \mathrm{Am}+\mathrm{d},{ }^{243} \mathrm{Am}+\mathrm{d},{ }^{244} \mathrm{Cm}+\mathrm{d},{ }^{245} \mathrm{Cm}+\mathrm{d},{ }^{246} \mathrm{Cm}, \\
{ }^{247} \mathrm{Cm}+\mathrm{d},{ }^{248} \mathrm{Cm}+\mathrm{d},{ }^{249} \mathrm{Cf}+\mathrm{d},{ }^{252} \mathrm{Cf}+\mathrm{d}\end{array}$ \\
\hline Slit Trenches & 4.8 & 1200 & $\begin{array}{l}{ }^{3} \mathrm{H},{ }^{14} \mathrm{C},{ }^{59} \mathrm{Ni},{ }^{79} \mathrm{Se},{ }^{87} \mathrm{Rb},{ }^{90} \mathrm{Sr}+\mathrm{d},{ }^{93} \mathrm{Zr}+\mathrm{d},{ }^{99} \mathrm{Tc}, \\
{ }^{107} \mathrm{Pd},{ }^{126} \mathrm{Sn}+\mathrm{d},{ }^{129} \mathrm{I},{ }^{135} \mathrm{Cs},{ }^{230} \mathrm{Th}+\mathrm{d},{ }^{232} \mathrm{Th}+\mathrm{d}, \\
{ }^{232} \mathrm{U}+\mathrm{d},{ }^{233} \mathrm{U}+\mathrm{d},{ }^{234} \mathrm{U}+\mathrm{d},{ }^{235} \mathrm{U}+\mathrm{d},{ }^{236} \mathrm{U},{ }^{237} \mathrm{~Np}, \\
{ }^{238} \mathrm{U}+\mathrm{d},{ }^{238} \mathrm{Pu}+\mathrm{d},{ }^{239} \mathrm{Pu}+\mathrm{d},{ }^{240} \mathrm{Pu}+\mathrm{d},{ }^{241} \mathrm{Pu}+\mathrm{d}, \\
{ }^{242} \mathrm{Pu}+\mathrm{d},{ }^{244} \mathrm{Pu},{ }^{241} \mathrm{Am}+\mathrm{d},{ }^{243} \mathrm{Am}+\mathrm{d},{ }^{242} \mathrm{Cm}+\mathrm{d}, \\
{ }^{244} \mathrm{Cm}+\mathrm{d},{ }^{245} \mathrm{Cm}+\mathrm{d},{ }^{246} \mathrm{Cm},{ }^{247} \mathrm{Cm}+\mathrm{d},{ }^{248} \mathrm{Cm}+\mathrm{d}, \\
{ }^{249} \mathrm{Bk}+\mathrm{d},{ }^{249} \mathrm{Cf}+\mathrm{d},{ }^{252} \mathrm{Cf}+\mathrm{d}\end{array}$ \\
\hline $\begin{array}{l}\text { Intimately- } \\
\text { mixed } \\
\text { Cement- } \\
\text { stabilized } \\
\text { Waste Units }\end{array}$ & 4.8 & 1200 & $\begin{array}{l}{ }^{3} \mathrm{H},{ }^{14} \mathrm{C},{ }^{59} \mathrm{Ni},{ }^{79} \mathrm{Se},{ }^{87} \mathrm{Rb},{ }^{90} \mathrm{Sr}+\mathrm{d},{ }^{93} \mathrm{Zr}+\mathrm{d},{ }^{94} \mathrm{Nb}, \\
{ }^{99} \mathrm{Tc},{ }^{107} \mathrm{Pd},{ }^{126} \mathrm{Sn}+\mathrm{d},{ }^{129} \mathrm{I},{ }^{135} \mathrm{Cs},{ }^{232} \mathrm{Th}+\mathrm{d}, \\
{ }^{232} \mathrm{U}+\mathrm{d},{ }^{233} \mathrm{U}+\mathrm{d},{ }^{234} \mathrm{U}+\mathrm{d},{ }^{235} \mathrm{U}+\mathrm{d},{ }^{236} \mathrm{U},{ }^{237} \mathrm{~Np}, \\
{ }^{238} \mathrm{U}+\mathrm{d},{ }^{238} \mathrm{Pu}+\mathrm{d},{ }^{239} \mathrm{Pu}+\mathrm{d},{ }^{240} \mathrm{Pu}+\mathrm{d},{ }^{24} \mathrm{Pu}+\mathrm{d}, \\
{ }^{242} \mathrm{Pu}+\mathrm{d},{ }^{244} \mathrm{Pu},{ }^{241} \mathrm{Am}+\mathrm{d},{ }^{243} \mathrm{Am}+\mathrm{d},{ }^{244} \mathrm{Cm}+\mathrm{d}, \\
{ }^{245} \mathrm{Cm}+\mathrm{d},{ }^{246} \mathrm{Cm},{ }^{247} \mathrm{Cm}+\mathrm{d},{ }^{248} \mathrm{Cm}+\mathrm{d},{ }^{249} \mathrm{Cf}+\mathrm{d}, \\
{ }^{252} \mathrm{Cf}+\mathrm{d}\end{array}$ \\
\hline $\begin{array}{l}\text { Cement- } \\
\text { stabilized } \\
\text { Encapsulated } \\
\text { Waste Units }\end{array}$ & 4.8 & 1200 & $\begin{array}{l}{ }^{3} \mathrm{H},{ }^{14} \mathrm{C},{ }^{59} \mathrm{Ni},{ }^{79} \mathrm{Se},{ }^{87} \mathrm{Rb},{ }^{90} \mathrm{Sr}+\mathrm{d},{ }^{93} \mathrm{Zr}+\mathrm{d},{ }^{99} \mathrm{Tc}, \\
{ }^{107} \mathrm{Pd},{ }^{126} \mathrm{Sn}+\mathrm{d},{ }^{129} \mathrm{I},{ }^{135} \mathrm{Cs},{ }^{232} \mathrm{Th}+\mathrm{d},{ }^{232} \mathrm{U}+\mathrm{d}, \\
{ }^{233} \mathrm{U}+\mathrm{d},{ }^{234} \mathrm{U}+\mathrm{d},{ }^{235} \mathrm{U}+\mathrm{d},{ }^{236} \mathrm{U},{ }^{237} \mathrm{~Np},{ }^{238} \mathrm{U}+\mathrm{d}, \\
{ }^{238} \mathrm{Pu}+\mathrm{d},{ }^{239} \mathrm{Pu}+\mathrm{d},{ }^{240} \mathrm{Pu}+\mathrm{d},{ }^{241} \mathrm{Pu}+\mathrm{d},{ }^{242} \mathrm{Pu}+\mathrm{d}, \\
{ }^{244} \mathrm{Pu},{ }^{241} \mathrm{Am}+\mathrm{d},{ }^{243} \mathrm{Am}+\mathrm{d},{ }^{242} \mathrm{Cm}+\mathrm{d},{ }^{244} \mathrm{Cm}+\mathrm{d}, \\
{ }^{245} \mathrm{Cm}+\mathrm{d},{ }^{246} \mathrm{Cm},{ }^{247} \mathrm{Cm}+\mathrm{d},{ }^{248} \mathrm{Cm}+\mathrm{d},{ }^{249} \mathrm{Bk}+\mathrm{d}, \\
{ }^{249} \mathrm{Cf}+\mathrm{d},{ }^{252} \mathrm{Cf}+\mathrm{d}\end{array}$ \\
\hline
\end{tabular}


Table 4.1-2 Parameters Used in Screening of Radionuclide Inventories for E-Area LowLevel Waste Facility Disposal Units and Lists of Radionuclides for Further Analysis with Respect to Off-Site Public Exposures

\begin{tabular}{|c|c|c|c|}
\hline $\begin{array}{l}\text { Type of } \\
\text { Disposal Unit }\end{array}$ & $\begin{array}{l}\text { Depth of } \\
\text { Waste in } \\
\text { Disposal } \\
\text { Unit (m) }\end{array}$ & $\begin{array}{l}\text { Area of } \\
\text { Waste in } \\
\text { Disposal } \\
\text { Unit }\left(\mathrm{m}^{2}\right)\end{array}$ & $\begin{array}{c}\text { Radionuclides Identified as Potentially } \\
\text { Significant Contributors to Dose Related to } \\
\text { Water Resource Impacts Analysis }\end{array}$ \\
\hline $\begin{array}{l}\text { Naval } \\
\text { Reactor } \\
\text { Components }\end{array}$ & 4.7 & $1850^{\mathrm{b}}$ & $\begin{array}{l}{ }^{3} \mathrm{H},{ }^{14} \mathrm{C},{ }^{59} \mathrm{Ni},{ }^{79} \mathrm{Se},{ }^{90} \mathrm{Sr}+\mathrm{d},{ }^{93} \mathrm{Zr}+\mathrm{d},{ }^{94} \mathrm{Nb},{ }^{99} \mathrm{Tc}, \\
{ }^{107} \mathrm{Pd},{ }^{126} \mathrm{Sn}+\mathrm{d},{ }^{129} \mathrm{I},{ }^{135} \mathrm{Cs},{ }^{232} \mathrm{Th}+\mathrm{d},{ }^{232} \mathrm{U}+\mathrm{d}, \\
{ }^{234} \mathrm{U}+\mathrm{d},{ }^{235} \mathrm{U}+\mathrm{d},{ }^{236} \mathrm{U},{ }^{237} \mathrm{~Np},{ }^{238} \mathrm{U}+\mathrm{d},{ }^{238} \mathrm{Pu}+\mathrm{d}, \\
{ }^{239} \mathrm{Pu}+\mathrm{d},{ }^{240} \mathrm{Pu}+\mathrm{d},{ }^{241} \mathrm{Pu}+\mathrm{d},{ }^{242} \mathrm{Pu}+\mathrm{d},{ }^{244} \mathrm{Pu}, \\
{ }^{241} \mathrm{Am}+\mathrm{d},{ }^{243} \mathrm{Am}+\mathrm{d},{ }^{244} \mathrm{Cm}+\mathrm{d},{ }^{245} \mathrm{Cm}+\mathrm{d},{ }^{246} \mathrm{Cm}, \\
{ }^{247} \mathrm{Cm}+\mathrm{d},{ }^{248} \mathrm{Cm}+\mathrm{d},{ }^{249} \mathrm{Cf}+\mathrm{d}\end{array}$ \\
\hline
\end{tabular}

Starting list of radionuclides (i.e., before screening) assumed to be the same as that for the LAW vaults.

b Corresponds to an area taken up by 100 casks.

\begin{tabular}{cccc}
\hline \multicolumn{4}{c}{ Table 4.2-2. Dose Factors for the Air Pathway } \\
\hline Disposal Unit & Radionuclide & $\begin{array}{c}\mathbf{1 0 0} \mathbf{~ m} \text { Location } \\
\text { (mrem/Ci Released) }\end{array}$ & $\begin{array}{c}\text { Site Boundary Location } \\
\text { (mrem/Ci Released) }\end{array}$ \\
\hline CIG Trenches & ${ }^{3} \mathrm{H}$ & $3.0 \mathrm{E}-03$ & $2.4 \mathrm{E}-05$ \\
& ${ }^{14} \mathrm{C}$ & $1.4 \mathrm{E}-01$ & $1.2 \mathrm{E}-03$ \\
Disposal Units & & & \\
other than CIG Trenches & & $8.5 \mathrm{E}-03$ & $2.4 \mathrm{E}-06$ \\
& ${ }^{3} \mathrm{H}$ & 3.8 & $1.0 \mathrm{E}-03$ \\
\hline
\end{tabular}

\begin{tabular}{c|l|l|l}
\hline Table 4.3-2. Dose for Unit Release via the Air Pathway & \\
\hline Disposal Unit & & $\begin{array}{l}\text { Dose for Unit Release } \\
(\mathrm{mrem} / \mathrm{Ci})\end{array}$ & \\
\hline & Radionuclide & 100 meters & SRS Boundary \\
\hline CIG Trenches & & & $3.0 \mathrm{E}-03$ \\
\hline & ${ }^{3} \mathrm{H}$ (oxide) & $1.4 \mathrm{E}-01$ & $2.4 \mathrm{E}-05$ \\
\hline $\begin{array}{c}\text { Disposal Units } \\
\text { other than CIG Trenches }\end{array}$ & ${ }^{14} \mathrm{C}$ & & $1.2 \mathrm{E}-03$ \\
\hline & ${ }^{3} \mathrm{H}$ (oxide) & $8.5 \mathrm{E}-03$ & \\
\hline & ${ }^{14} \mathrm{C}$ & 3.8 & $2.4 \mathrm{E}-06$ \\
\hline
\end{tabular}


Table 5.2-1. Results of the Air Pathway Analysis

\begin{tabular}{|c|c|c|c|}
\hline & $\begin{array}{c}\text { Inventory Limit } \\
\text { Based on } \\
\text { Location } 100 \mathrm{~m} \\
\text { from Waste after } \\
100 \text { Year } \\
\text { Institution } \\
\text { Control Period } \\
\text { (Ci/disposal Unit) }\end{array}$ & $\begin{array}{c}\text { Inventory Limit } \\
\text { Based on Location } \\
\text { at SRS Boundary } \\
\text { before } 100 \text { year } \\
\text { Institution Control } \\
\text { Period } \\
\text { (Ci/disposal } \\
\text { Unit) }\end{array}$ & $\begin{array}{c}\text { Inventory Limit } \\
\text { Based on Air } \\
\text { Pathway } \\
\text { (Ci/disposal Unit) }\end{array}$ \\
\hline \multicolumn{4}{|l|}{$\mathrm{H}-3$} \\
\hline 5 Trenches $^{\mathrm{a}, \mathrm{b}}$ & $3.2 \mathrm{E}+05$ & $4.2 \mathrm{E}+06$ & $3.2 \mathrm{E}+05$ \\
\hline 5 CSEW Trenches & $9.0 \mathrm{E}+05$ & $4.1 \mathrm{E}+05$ & $4.1 \mathrm{E}+05$ \\
\hline Crucibles (1 vault) & $4.1 \mathrm{E}+13$ & $5.2 \mathrm{E}+14$ & $4.1 \mathrm{E}+13$ \\
\hline 1 LAW Vault JCW & $6.4 \mathrm{E}+07$ & $8.2 \mathrm{E}+08$ & $6.4 \mathrm{E}+07$ \\
\hline $1 \mathrm{IL}$ Vault JCW & $5.5 \mathrm{E}+07$ & $7.1 \mathrm{E}+08$ & $5.5 \mathrm{E}+07$ \\
\hline \multicolumn{4}{|l|}{$\mathrm{C}-14$} \\
\hline 5 Trenches $^{\mathrm{a}}$ & 2.7 & $1.0 \mathrm{E}+04$ & 2.7 \\
\hline 5 CSEW Trenches & $7.0 \mathrm{E}+01$ & $8.7 \mathrm{E}+03$ & $7.0 \mathrm{E}+01$ \\
\hline 1 LAW Vault JCW & 2.7 & $1.0 \mathrm{E}+04$ & 2.7 \\
\hline $1 \mathrm{IL}$ Vault JCW & 2.7 & $1.0 \mathrm{E}+04$ & 2.7 \\
\hline \multicolumn{4}{|l|}{$\mathrm{U}-234$ based on $\mathrm{Rn}-222^{\mathrm{c}}$} \\
\hline 5 Trenches $^{\mathrm{a}}$ & 49 & & 49 \\
\hline 1 LAW Vault & 125 & & 125 \\
\hline $1 \mathrm{IL}$ Vault & 15 & & 15 \\
\hline
\end{tabular}

${ }^{\text {a }}$ Includes Intimately Mixed Cement Based Wasteforms

${ }^{\mathrm{b}}$ Limits also used for NR waste pad

${ }^{\mathrm{c}}$ Based on radon flux limit of $20 \mathrm{pCi} / \mathrm{m} 2-\mathrm{sec}$ 
Table 4.3-7. Estimated Peak Fractional Flux to the Water Table for Radionuclides Disposed of in E-Area Cement-Stabilized Encapsulated Waste

\begin{tabular}{|c|c|c|}
\hline Radionuclide & $\begin{array}{c}\text { Peak Flux to water table } \\
\mathrm{Ci} / \mathrm{yr}\end{array}$ & $\begin{array}{c}\text { Time of peak flux } \\
\text { yr }\end{array}$ \\
\hline H-3 & $1.05 \mathrm{E}-08$ & $3.05 \mathrm{E}+02$ \\
\hline C-14 & 4.92E-04 & $3.90 \mathrm{E}+02$ \\
\hline C-14_KB & $4.22 \mathrm{E}-04$ & $7.60 \mathrm{E}+02$ \\
\hline $\mathrm{Ni}-59$ & $1.75 \mathrm{E}-04$ & $8.54 \mathrm{E}+03$ \\
\hline Se-79 & $5.49 \mathrm{E}-04$ & $2.99 \mathrm{E}+03$ \\
\hline $\mathrm{Rb}-87$ & $1.54 \mathrm{E}-03$ & $1.29 \mathrm{E}+03$ \\
\hline Sr-90 & $1.43 \mathrm{E}-07$ & $4.38 \mathrm{E}+02$ \\
\hline $\mathrm{Zr}-93$ & $8.27 \mathrm{E}-05$ & $1.00 \mathrm{E}+04$ \\
\hline $\mathrm{Nb}-93 \mathrm{~m}$ & $3.09 \mathrm{E}-04$ & $1.00 \mathrm{E}+04$ \\
\hline Tc-99 & $1.32 \mathrm{E}-01$ & $3.12 \mathrm{E}+02$ \\
\hline Tc-99_KB & $5.37 \mathrm{E}-04$ & $1.07 \mathrm{E}+03$ \\
\hline Pd-107 & $1.53 \mathrm{E}-03$ & $1.32 \mathrm{E}+03$ \\
\hline Sn-126 & $5.65 \mathrm{E}-04$ & $2.82 \mathrm{E}+03$ \\
\hline I- 129 & $9.53 \mathrm{E}-02$ & $3.17 \mathrm{E}+02$ \\
\hline I-129_A & $1.36 \mathrm{E}-03$ & $6.17 \mathrm{E}+02$ \\
\hline I-129_B & $4.96 \mathrm{E}-04$ & $1.15 \mathrm{E}+03$ \\
\hline I-129_C & 7.27E-04 & $8.79 \mathrm{E}+02$ \\
\hline I-129_D & $1.56 \mathrm{E}-04$ & $2.89 \mathrm{E}+03$ \\
\hline I-129_E & $2.20 \mathrm{E}-04$ & $2.14 \mathrm{E}+03$ \\
\hline I-129_F & $6.93 \mathrm{E}-04$ & $9.07 \mathrm{E}+02$ \\
\hline I-129_G & 7.94E-02 & $3.20 \mathrm{E}+02$ \\
\hline I-129_H & 4.37E-03 & $4.15 \mathrm{E}+02$ \\
\hline I-129_I & $1.41 \mathrm{E}-04$ & $3.15 \mathrm{E}+03$ \\
\hline I-129_J & $3.31 \mathrm{E}-02$ & $3.31 \mathrm{E}+02$ \\
\hline I-129_KB & $1.18 \mathrm{E}-04$ & $3.80 \mathrm{E}+03$ \\
\hline I-129_S & 7.57E-05 & $5.65 \mathrm{E}+03$ \\
\hline I-129_10K & 4.37E-05 & $1.00 \mathrm{E}+04$ \\
\hline Cs -135 & $2.59 \mathrm{E}-04$ & $6.18 \mathrm{E}+03$ \\
\hline Th-232 & $5.41 \mathrm{E}-13$ & $1.00 \mathrm{E}+04$ \\
\hline $\mathrm{Ra}-228$ & $3.73 \mathrm{E}-12$ & $1.00 \mathrm{E}+04$ \\
\hline Th-228 & $5.81 \mathrm{E}-13$ & $1.00 \mathrm{E}+04$ \\
\hline $\mathrm{Ra}-224$ & $3.72 \mathrm{E}-12$ & $1.00 \mathrm{E}+04$ \\
\hline U-232 & $6.64 \mathrm{E}-10$ & $8.88 \mathrm{E}+02$ \\
\hline Th-228 & 7.32E- 12 & $8.88 \mathrm{E}+02$ \\
\hline $\mathrm{Ra}-224$ & $4.69 \mathrm{E}-11$ & $8.88 \mathrm{E}+02$ \\
\hline U-233 & 7.11E-05 & $6.91 \mathrm{E}+03$ \\
\hline Th-229 & 4.13E-07 & $1.00 \mathrm{E}+04$ \\
\hline $\mathrm{Ra}-225$ & $2.71 \mathrm{E}-06$ & $1.00 \mathrm{E}+04$ \\
\hline $\mathrm{U}-234$ & $7.19 \mathrm{E}-05$ & $7.03 \mathrm{E}+03$ \\
\hline Th-230 & $5.64 \mathrm{E}-08$ & $1.00 \mathrm{E}+04$ \\
\hline Ra-226 & $6.11 \mathrm{E}-07$ & $1.00 \mathrm{E}+04$ \\
\hline $\mathrm{Pb}-210$ & $1.13 \mathrm{E}-06$ & $1.00 \mathrm{E}+04$ \\
\hline Po-210 & $2.04 \mathrm{E}-06$ & $1.00 \mathrm{E}+04$ \\
\hline U-235 & 7.33E-05 & $7.05 \mathrm{E}+03$ \\
\hline $\mathrm{Pa}-231$ & $4.82 \mathrm{E}-06$ & $1.00 \mathrm{E}+04$ \\
\hline Ac-227 & $5.93 \mathrm{E}-06$ & $1.00 \mathrm{E}+04$ \\
\hline Th-227 & 8.35E-07 & $1.00 \mathrm{E}+04$ \\
\hline
\end{tabular}


Table 4.3-7. Estimated Peak Fractional Flux to the Water Table for Radionuclides Disposed of in E-Area Cement-Stabilized Encapsulated Waste

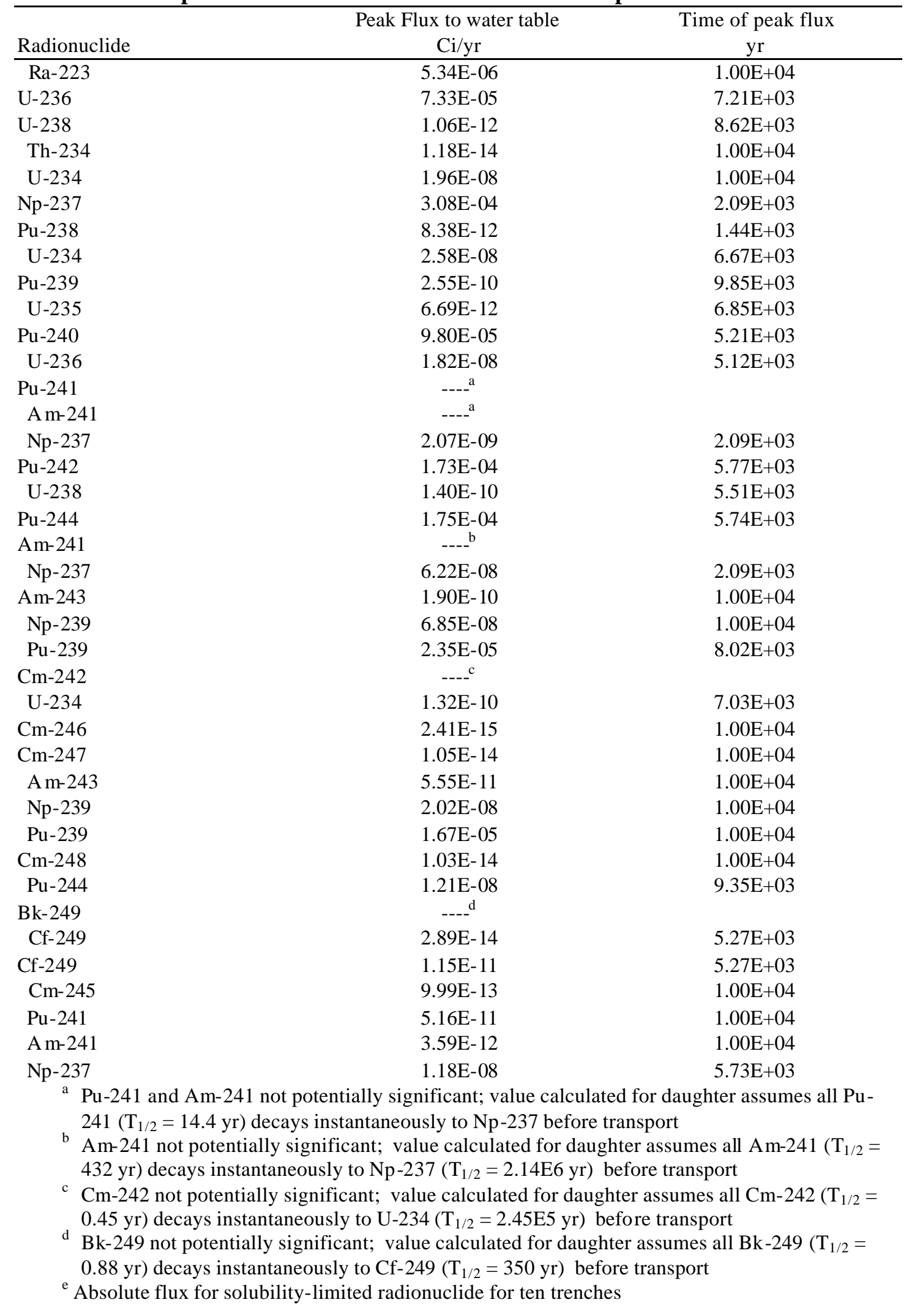


Table 5.1-7. Peak Groundwater Concentrations for the Cement-Stabilized Encapsulated Waste Simulations - per Ci of Each Radionuclide in 10 Trenches

\begin{tabular}{|c|c|c|c|}
\hline Radionuclide & $\begin{array}{l}\text { Peak up to } 10,000 \text { years }{ }^{a} \\
\text { pCi/L }\end{array}$ & $\begin{array}{c}\text { Peak after 10,000 years } \\
\text { pCi/L-Ci }\end{array}$ & $\begin{array}{c}\text { Time of peak } \\
\mathrm{yr}\end{array}$ \\
\hline$\overline{\mathrm{H}-3}$ & $1.16 \mathrm{E}-04$ & TBD & $3.09 \mathrm{E}+02$ \\
\hline C- 14 & $1.77 \mathrm{E}+01$ & TBD & $6.39 \mathrm{E}+02$ \\
\hline C-14_KB & $1.62 \mathrm{E}+01$ & TBD & $9.09 \mathrm{E}+02$ \\
\hline $\mathrm{Ni}-59$ & $1.61 \mathrm{E}-01$ & TBD & $1.00 \mathrm{E}+04$ \\
\hline $\mathrm{Se}-79$ & $3.78 \mathrm{E}+00$ & TBD & $7.05 \mathrm{E}+03$ \\
\hline $\mathrm{Rb}-87$ & $1.11 \mathrm{E}+01$ & TBD & $2.81 \mathrm{E}+03$ \\
\hline Sr-90 & $2.54 \mathrm{E}-05$ & TBD & $5.34 \mathrm{E}+02$ \\
\hline Zr-93 & $3.23 \mathrm{E}-03$ & TBD & $1.00 \mathrm{E}+04$ \\
\hline $\mathrm{Nb}-93 \mathrm{~m}$ & $1.26 \mathrm{E}-02$ & TBD & $1.00 \mathrm{E}+04$ \\
\hline Tc-99 & $1.20 \mathrm{E}+03$ & TBD & $3.26 \mathrm{E}+02$ \\
\hline Tc-99_KB & $2.15 \mathrm{E}+01$ & TBD & $1.10 \mathrm{E}+03$ \\
\hline Pd-107 & $1.11 \mathrm{E}+01$ & TBD & $2.83 \mathrm{E}+03$ \\
\hline Sn-126 & $4.43 \mathrm{E}+00$ & TBD & $6.44 \mathrm{E}+03$ \\
\hline I- 129 & $8.15 \mathrm{E}+02$ & TBD & $3.37 \mathrm{E}+02$ \\
\hline I-129_A & $5.45 \mathrm{E}+01$ & TBD & $6.62 \mathrm{E}+02$ \\
\hline I-129_B & $1.98 \mathrm{E}+01$ & TBD & $1.19 \mathrm{E}+03$ \\
\hline I-129_C & $2.91 \mathrm{E}+01$ & TBD & $9.27 \mathrm{E}+02$ \\
\hline I-129_D & $6.23 \mathrm{E}+00$ & TBD & $3.00 \mathrm{E}+03$ \\
\hline I-129_E & $8.81 \mathrm{E}+00$ & TBD & $2.23 \mathrm{E}+03$ \\
\hline I-129_F & $2.77 \mathrm{E}+01$ & TBD & $9.55 \mathrm{E}+02$ \\
\hline I-129_G & $8.01 \mathrm{E}+02$ & TBD & $3.40 \mathrm{E}+02$ \\
\hline I-129_H & $1.73 \mathrm{E}+02$ & TBD & $4.55 \mathrm{E}+02$ \\
\hline I-129_I & $5.63 \mathrm{E}+00$ & TBD & $3.28 \mathrm{E}+03$ \\
\hline I-129_J & $6.75 \mathrm{E}+02$ & TBD & $3.66 \mathrm{E}+02$ \\
\hline I-129_KB & $4.71 \mathrm{E}+00$ & TBD & $3.85 \mathrm{E}+03$ \\
\hline I-129_S & $3.03 \mathrm{E}+00$ & TBD & $5.80 \mathrm{E}+03$ \\
\hline I-129_10K & $1.74 \mathrm{E}+00$ & TBD & $9.77 \mathrm{E}+03$ \\
\hline Cs -135 & $8.87 \mathrm{E}-01$ & TBD & $1.00 \mathrm{E}+04$ \\
\hline Th-232 & $2.59 \mathrm{E}-15$ & TBD & $1.00 \mathrm{E}+04$ \\
\hline Ra-228 & $1.83 \mathrm{E}-14$ & TBD & $1.00 \mathrm{E}+04$ \\
\hline Th-228 & $2.84 \mathrm{E}-15$ & TBD & $1.00 \mathrm{E}+04$ \\
\hline Ra-224 & $1.82 \mathrm{E}-14$ & TBD & $1.00 \mathrm{E}+04$ \\
\hline U-232 & $6.62 \mathrm{E}-08$ & TBD & $1.18 \mathrm{E}+03$ \\
\hline Th-228 & $7.27 \mathrm{E}-10$ & TBD & $1.18 \mathrm{E}+03$ \\
\hline Ra-224 & $4.66 \mathrm{E}-09$ & TBD & $1.18 \mathrm{E}+03$ \\
\hline $\mathrm{U}-233$ & $2.79 \mathrm{E}+00$ & TBD & $9.17 \mathrm{E}+03$ \\
\hline Th-229 & $1.32 \mathrm{E}-02$ & TBD & $1.00 \mathrm{E}+04$ \\
\hline Ra-225 & $8.40 \mathrm{E}-02$ & TBD & $1.00 \mathrm{E}+04$ \\
\hline $\mathrm{U}-234$ & $2.83 \mathrm{E}+00$ & TBD & $9.28 \mathrm{E}+03$ \\
\hline Th-230 & $1.67 \mathrm{E}-03$ & TBD & $1.00 \mathrm{E}+04$ \\
\hline Ra-226 & $7.21 \mathrm{E}-03$ & TBD & $1.00 \mathrm{E}+04$ \\
\hline $\mathrm{Pb}-210$ & $1.33 \mathrm{E}-02$ & TBD & $1.00 \mathrm{E}+04$ \\
\hline Po-210 & 2.39E-02 & TBD & $1.00 \mathrm{E}+04$ \\
\hline $\mathrm{U}-235$ & $2.91 \mathrm{E}+00$ & TBD & $9.48 \mathrm{E}+03$ \\
\hline $\mathrm{Pa}-231$ & $2.35 \mathrm{E}-02$ & TBD & $1.00 \mathrm{E}+04$ \\
\hline Ac- 227 & $2.87 \mathrm{E}-02$ & TBD & $1.00 \mathrm{E}+04$ \\
\hline Th-227 & $4.03 \mathrm{E}-03$ & TBD & $1.00 \mathrm{E}+04$ \\
\hline
\end{tabular}


Table 5.1-7. Peak Groundwater Concentrations for the Cement-Stabilized Encapsulated Waste Simulations - per Ci of Each Radionuclide in 10 Trenches

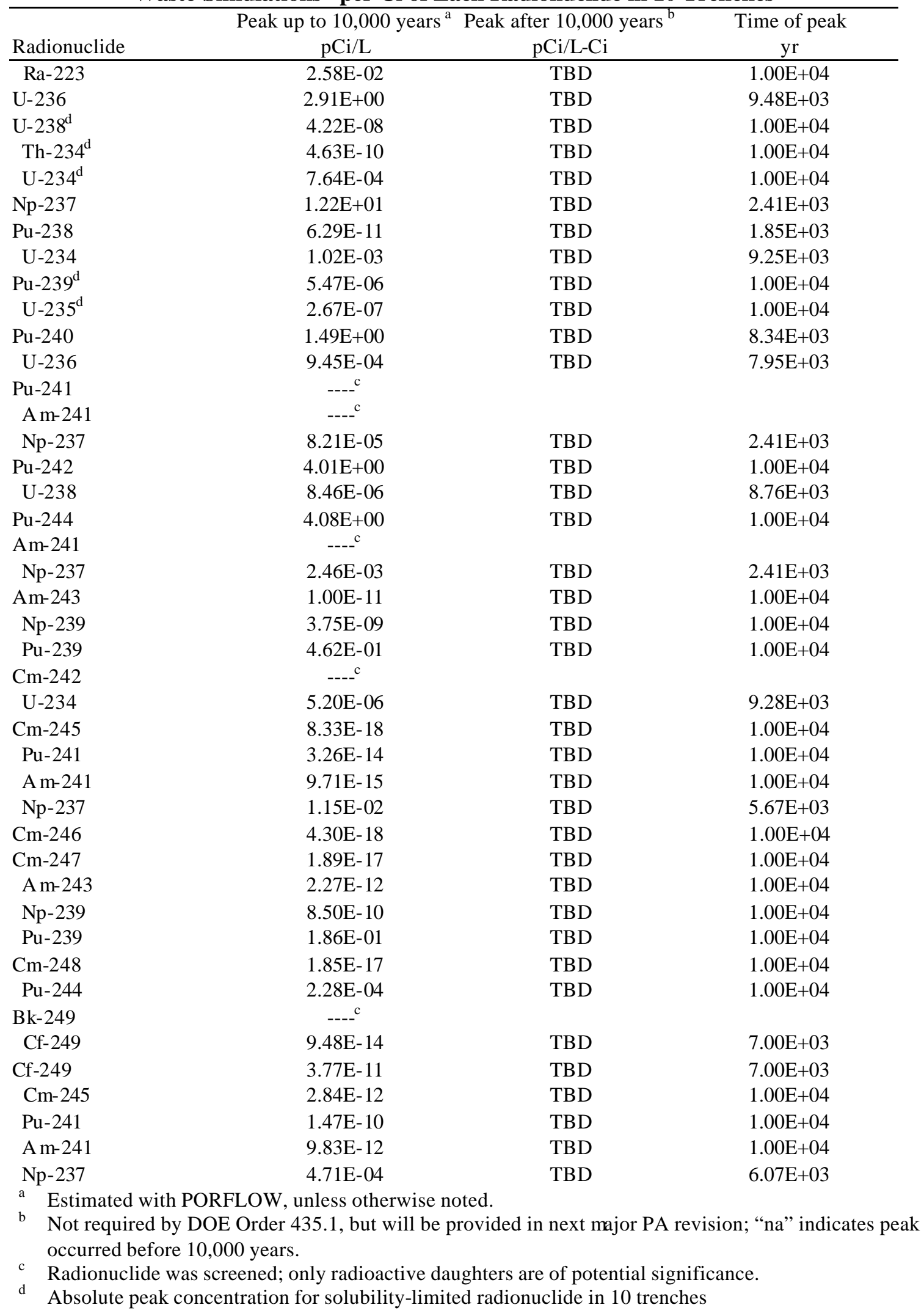


Table 5.1-13. Calculated Inventory Limits for the Trenches Containing Cement-Stabilized Encapsulated Waste

\begin{tabular}{|c|c|c|c|c|}
\hline Radionuclide $^{\mathrm{a}}$ & $\begin{array}{c}\text { Concentration } \\
\text { limit }^{\mathrm{b}}\end{array}$ & $\begin{array}{c}\text { Peak } \\
\text { groundwater } \\
\text { concentration up } \\
\text { to } 10,000 \text { years } \\
\text { pCi/L }\end{array}$ & $\begin{array}{c}\text { Calculated } \\
\text { inventory } \\
\text { limit }^{\mathrm{d}}\end{array}$ & $\begin{array}{c}\text { Maximum } \\
\text { fraction if } \\
\text { solubility-limited }\end{array}$ \\
\hline H-3 & $2.00 \mathrm{E}+04$ & $1.16 \mathrm{E}-04$ & $8.62 \mathrm{E}+07$ & \\
\hline C-14 & $2.00 \mathrm{E}+03$ & $1.77 \mathrm{E}+01$ & $5.65 \mathrm{E}+01$ & \\
\hline C-14_KB & $2.00 \mathrm{E}+03$ & $1.62 \mathrm{E}+01$ & $6.17 \mathrm{E}+01$ & \\
\hline $\mathrm{Ni}-59$ & $3.00 \mathrm{E}+02$ & $1.61 \mathrm{E}-01$ & $9.32 \mathrm{E}+02$ & \\
\hline Se-79 & $7.00 \mathrm{E}+02$ & $3.78 \mathrm{E}+00$ & $9.26 \mathrm{E}+01$ & \\
\hline $\mathrm{Rb}-87$ & $3.00 \mathrm{E}+02$ & $1.11 \mathrm{E}+01$ & $1.35 \mathrm{E}+01$ & \\
\hline Sr-90 & $8.00 \mathrm{E}+00$ & $2.54 \mathrm{E}-05$ & $1.57 \mathrm{E}+05$ & \\
\hline $\mathrm{Zr}-93$ & $2.00 \mathrm{E}+03$ & $3.23 \mathrm{E}-03$ & $3.10 \mathrm{E}+05$ & \\
\hline $\mathrm{Nb}-93 \mathrm{~m}$ & $1.00 \mathrm{E}+03$ & $1.26 \mathrm{E}-02$ & $3.97 \mathrm{E}+04$ & \\
\hline Tc-99 & $9.00 \mathrm{E}+02$ & $1.20 \mathrm{E}+03$ & $3.75 \mathrm{E}-01$ & \\
\hline Tc-99_KB & $9.00 \mathrm{E}+02$ & $2.15 \mathrm{E}+01$ & $2.09 \mathrm{E}+01$ & \\
\hline Pd-107 & $4.00 \mathrm{E}+04$ & $1.11 \mathrm{E}+01$ & $1.80 \mathrm{E}+03$ & \\
\hline Sn-126 & $3.00 \mathrm{E}+02$ & $4.43 \mathrm{E}+00$ & $3.39 \mathrm{E}+01$ & \\
\hline I- 129 & $1.00 \mathrm{E}+00$ & $8.15 \mathrm{E}+02$ & $6.13 \mathrm{E}-04$ & \\
\hline I-129_A & $1.00 \mathrm{E}+00$ & $5.45 \mathrm{E}+01$ & $9.17 \mathrm{E}-03$ & \\
\hline I-129_B & $1.00 \mathrm{E}+00$ & $1.98 \mathrm{E}+01$ & $2.53 \mathrm{E}-02$ & \\
\hline I-129_C & $1.00 \mathrm{E}+00$ & $2.91 \mathrm{E}+01$ & $1.72 \mathrm{E}-02$ & \\
\hline I-129_D & $1.00 \mathrm{E}+00$ & $6.23 \mathrm{E}+00$ & $8.03 \mathrm{E}-02$ & \\
\hline I-129_E & $1.00 \mathrm{E}+00$ & $8.81 \mathrm{E}+00$ & $5.68 \mathrm{E}-02$ & \\
\hline I-129_F & $1.00 \mathrm{E}+00$ & $2.77 \mathrm{E}+01$ & $1.81 \mathrm{E}-02$ & \\
\hline I-129_G & $1.00 \mathrm{E}+00$ & $8.01 \mathrm{E}+02$ & $6.24 \mathrm{E}-04$ & \\
\hline I-129_H & $1.00 \mathrm{E}+00$ & $1.73 \mathrm{E}+02$ & $2.89 \mathrm{E}-03$ & \\
\hline I-129_I & $1.00 \mathrm{E}+00$ & $5.63 \mathrm{E}+00$ & $8.88 \mathrm{E}-02$ & \\
\hline I-129_J & $1.00 \mathrm{E}+00$ & $6.75 \mathrm{E}+02$ & 7.41E-04 & \\
\hline I-129_KB & $1.00 \mathrm{E}+00$ & $4.71 \mathrm{E}+00$ & $1.06 \mathrm{E}-01$ & \\
\hline I-129_S & $1.00 \mathrm{E}+00$ & $3.03 \mathrm{E}+00$ & $1.65 \mathrm{E}-01$ & \\
\hline I-129_10K & $1.00 \mathrm{E}+00$ & $1.74 \mathrm{E}+00$ & $2.87 \mathrm{E}-01$ & \\
\hline Cs -135 & $9.00 \mathrm{E}+02$ & $8.87 \mathrm{E}-01$ & $5.07 \mathrm{E}+02$ & \\
\hline Th-232 & $1.30 \mathrm{E}+01$ & $2.59 \mathrm{E}-15$ & $2.51 \mathrm{E}+15$ & \\
\hline Ra-228 & $5.00 \mathrm{E}+00$ & $1.83 \mathrm{E}-14$ & $1.37 \mathrm{E}+14$ & \\
\hline Th-228 & $1.50 \mathrm{E}+01$ & $2.84 \mathrm{E}-15$ & $2.64 \mathrm{E}+15$ & \\
\hline $\mathrm{Ra}-224$ & $1.50 \mathrm{E}+01$ & $1.82 \mathrm{E}-14$ & $4.12 \mathrm{E}+14$ & \\
\hline U-232 & $2.60 \mathrm{E}+01$ & $6.62 \mathrm{E}-08$ & $1.96 \mathrm{E}+08$ & \\
\hline Th-228 & $1.50 \mathrm{E}+01$ & $7.27 \mathrm{E}-10$ & $1.03 \mathrm{E}+10$ & \\
\hline Ra-224 & $1.50 \mathrm{E}+01$ & $4.66 \mathrm{E}-09$ & $1.61 \mathrm{E}+09$ & \\
\hline U-233 & $1.30 \mathrm{E}+02$ & $2.79 \mathrm{E}+00$ & $2.33 E+01$ & \\
\hline Th-229 & $9.60 \mathrm{E}+00$ & $1.32 \mathrm{E}-02$ & $3.64 \mathrm{E}+02$ & \\
\hline Ra-225 & $2.00 \mathrm{E}+01$ & $8.40 \mathrm{E}-02$ & $1.19 \mathrm{E}+02$ & \\
\hline U-234 & $1.30 \mathrm{E}+02$ & $2.83 \mathrm{E}+00$ & $2.30 \mathrm{E}+01$ & \\
\hline Th-230 & $1.50 \mathrm{E}+01$ & $1.67 \mathrm{E}-03$ & $4.49 \mathrm{E}+03$ & \\
\hline Ra-226 & $5.00 \mathrm{E}+00$ & $7.21 \mathrm{E}-03$ & $3.47 \mathrm{E}+02$ & \\
\hline $\mathrm{Pb}-210$ & $1.00 \mathrm{E}+00$ & $1.33 \mathrm{E}-02$ & $3.76 \mathrm{E}+01$ & \\
\hline Po-210 & $1.50 \mathrm{E}+01$ & 2.39E-02 & $3.14 \mathrm{E}+02$ & \\
\hline U-235 & $6.50 \mathrm{E}+01$ & $2.91 \mathrm{E}+00$ & $1.12 \mathrm{E}+01$ & \\
\hline
\end{tabular}


Table 5.1-13. Calculated Inventory Limits for the Trenches Containing Cement-Stabilized Encapsulated Waste

\begin{tabular}{|c|c|c|c|c|}
\hline Radionuclide $^{\text {a }}$ & $\begin{array}{c}\text { Concentration } \\
\text { limit }^{\mathrm{b}}\end{array}$ & $\begin{array}{c}\text { Peak } \\
\text { groundwater } \\
\text { concentration up } \\
\text { to } 10,000 \text { years } \\
\text { pCi/L }\end{array}$ & $\begin{array}{c}\text { Calculated } \\
\text { inventory } \\
\text { limit }^{\mathrm{d}}\end{array}$ & $\begin{array}{c}\text { Maximum } \\
\text { fraction if } \\
\text { solubility-limited }\end{array}$ \\
\hline $\mathrm{Pa}-231$ & $3.10 \mathrm{E}+00$ & $2.35 \mathrm{E}-02$ & $6.60 \mathrm{E}+01$ & \\
\hline Ac- -227 & $1.00 \mathrm{E}+00$ & $2.87 \mathrm{E}-02$ & $1.74 \mathrm{E}+01$ & \\
\hline Th-227 & $1.50 \mathrm{E}+01$ & $4.03 \mathrm{E}-03$ & $1.86 \mathrm{E}+03$ & \\
\hline Ra-223 & $1.50 \mathrm{E}+01$ & $2.58 \mathrm{E}-02$ & $2.91 \mathrm{E}+02$ & \\
\hline U-236 & $1.40 \mathrm{E}+02$ & $2.91 \mathrm{E}+00$ & $2.41 \mathrm{E}+01$ & \\
\hline $\mathrm{U}-238^{\mathrm{g}}$ & $1.00 \mathrm{E}+01$ & 4.22E-08 & $5.00 \mathrm{E}-03$ & 4.22E-09 \\
\hline Th- $234^{\mathrm{g}}$ & $4.00 \mathrm{E}+02$ & $4.63 \mathrm{E}-10$ & --- & $1.16 \mathrm{E}-12$ \\
\hline $\mathrm{U}-234^{\mathrm{g}}$ & $1.30 \mathrm{E}+02$ & 7.64E-04 & --- & $5.88 \mathrm{E}-06$ \\
\hline $\mathrm{Np}-237$ & $8.90 \mathrm{E}+00$ & $1.22 \mathrm{E}+01$ & $3.65 \mathrm{E}-01$ & \\
\hline $\mathrm{Pu}-238$ & $8.90 \mathrm{E}+00$ & $6.29 \mathrm{E}-11$ & 7.07E+10 & \\
\hline U-234 & $1.30 \mathrm{E}+02$ & $1.02 \mathrm{E}-03$ & $6.37 \mathrm{E}+04$ & \\
\hline $\mathrm{Pu}-239^{\mathrm{g}}$ & $8.10 \mathrm{E}+00$ & $5.47 \mathrm{E}-06$ & 4.60E-01 & 6.75E-07 \\
\hline $\mathrm{U}-235^{\mathrm{g}}$ & $6.50 \mathrm{E}+01$ & $2.67 \mathrm{E}-07$ & --- & $4.11 \mathrm{E}-09$ \\
\hline $\mathrm{Pu}-240$ & $8.10 \mathrm{E}+00$ & $1.49 \mathrm{E}+00$ & $2.72 \mathrm{E}+00$ & \\
\hline U-236 & $1.40 \mathrm{E}+02$ & $9.45 \mathrm{E}-04$ & $7.41 \mathrm{E}+04$ & \\
\hline $\mathrm{Pu}-241$ & & Screened & & \\
\hline A m-241 & & Screened & & \\
\hline $\mathrm{Np}-237$ & $8.90 \mathrm{E}+00$ & $8.21 \mathrm{E}-05^{\mathrm{f}}$ & $5.42 \mathrm{E}+04$ & \\
\hline $\mathrm{Pu}-242$ & $8.30 \mathrm{E}+00$ & $4.01 \mathrm{E}+00$ & $1.03 E+00$ & \\
\hline U-238 & $1.00 \mathrm{E}+01$ & $8.46 \mathrm{E}-06$ & $5.91 \mathrm{E}+05$ & \\
\hline $\mathrm{Pu}-244$ & $8.60 \mathrm{E}+00$ & $4.08 \mathrm{E}+00$ & $1.05 \mathrm{E}+00$ & \\
\hline Am-241 & & Screened & & \\
\hline Np-237 & $8.90 \mathrm{E}+00$ & $2.46 \mathrm{E}-03^{\mathrm{f}}$ & $1.81 E+03$ & \\
\hline Am-243 & $7.60 \mathrm{E}+00$ & $1.00 \mathrm{E}-11$ & $3.80 \mathrm{E}+11$ & \\
\hline $\mathrm{Np}-239$ & $3.00 \mathrm{E}+02$ & $3.75 \mathrm{E}-09$ & $4.00 \mathrm{E}+10$ & \\
\hline $\mathrm{Pu}-239$ & $8.10 \mathrm{E}+00$ & $4.62 \mathrm{E}-01$ & $8.77 \mathrm{E}+00$ & \\
\hline $\mathrm{Cm}-242$ & & Screened & & \\
\hline U-234 & $1.30 \mathrm{E}+02$ & $5.20 \mathrm{E}-06^{\mathrm{f}}$ & $1.25 \mathrm{E}+07$ & \\
\hline $\mathrm{Cm}-245$ & $7.60 \mathrm{E}+00$ & 8.33E-18 & $4.56 \mathrm{E}+17$ & \\
\hline $\mathrm{Pu}-241$ & $3.00 \mathrm{E}+02$ & $3.26 \mathrm{E}-14$ & $4.60 \mathrm{E}+15$ & \\
\hline A m-241 & $7.60 \mathrm{E}+00$ & $9.71 \mathrm{E}-15$ & $3.91 \mathrm{E}+14$ & \\
\hline Np-237 & $8.90 \mathrm{E}+00$ & $1.15 \mathrm{E}-02$ & $3.87 \mathrm{E}+02$ & \\
\hline $\mathrm{Cm}-246$ & $7.60 \mathrm{E}+00$ & $4.30 \mathrm{E}-18$ & $8.84 \mathrm{E}+17$ & \\
\hline $\mathrm{Cm}-247$ & $8.30 \mathrm{E}+00$ & $1.89 \mathrm{E}-17$ & $2.20 \mathrm{E}+17$ & \\
\hline A m-243 & $7.60 \mathrm{E}+00$ & $2.27 \mathrm{E}-12$ & $1.67 \mathrm{E}+12$ & \\
\hline Np-239 & $3.00 \mathrm{E}+02$ & $8.50 \mathrm{E}-10$ & $1.76 \mathrm{E}+11$ & \\
\hline $\mathrm{Pu}-239$ & $8.10 \mathrm{E}+00$ & $1.86 \mathrm{E}-01$ & $2.18 \mathrm{E}+01$ & \\
\hline $\mathrm{Cm}-248$ & $2.10 \mathrm{E}+00$ & $1.85 \mathrm{E}-17$ & $5.68 \mathrm{E}+16$ & \\
\hline $\mathrm{Pu}-244$ & $8.60 \mathrm{E}+00$ & $2.28 \mathrm{E}-04$ & 1.89E+04 & \\
\hline Bk-249 & & Screened & & \\
\hline Cf-249 & $7.40 \mathrm{E}+00$ & $9.48 \mathrm{E}-14^{\mathrm{f}}$ & $3.90 \mathrm{E}+13$ & \\
\hline Cf-249 & $7.40 \mathrm{E}+00$ & $3.77 \mathrm{E}-11$ & $9.81 \mathrm{E}+10$ & \\
\hline $\mathrm{Cm}-245$ & $7.60 \mathrm{E}+00$ & $2.84 \mathrm{E}-12$ & $1.34 \mathrm{E}+12$ & \\
\hline $\mathrm{Pu}-241$ & $3.00 \mathrm{E}+02$ & $1.47 \mathrm{E}-10$ & $1.02 \mathrm{E}+12$ & \\
\hline A m-241 & $7.60 \mathrm{E}+00$ & $9.83 \mathrm{E}-12$ & $3.87 \mathrm{E}+11$ & \\
\hline
\end{tabular}


Table 5.1-13. Calculated Inventory Limits for the Trenches Containing Cement-Stabilized Encapsulated Waste

\begin{tabular}{ccccc}
\hline & $\begin{array}{c}\text { Concentration } \\
\text { limit }^{\mathrm{b}}\end{array}$ & $\begin{array}{c}\text { Peak } \\
\text { groundwater } \\
\text { concentration up } \\
\text { to 10,000 years }\end{array}$ & $\begin{array}{c}\text { Calculated } \\
\text { inventory }^{\mathrm{limit}}{ }^{\mathrm{d}}\end{array}$ & $\begin{array}{c}\text { Maximum } \\
\text { fraction if } \\
\text { solubility-limited }\end{array}$ \\
Radionuclide $^{\mathrm{a}}$ & $\mathrm{pCi} / \mathrm{L}$ & $\mathrm{pCi} / \mathrm{L}$ & $\mathrm{Ci} / 5$ trenches & \\
\hline Np-237 & $8.90 \mathrm{E}+00$ & $4.71 \mathrm{E}-04$ & $\mathbf{9 . 4 5 E}+\mathbf{0 3}$ &
\end{tabular}

Note: Values in this table are rounded to the appropriate number of significant digits using Microsoft Excel 97 SR-2 (h)

a " $+\mathrm{d}$ " indicates short- and/or long-lived radioactive daughters are considered in the analysis.

b The more restrictive of either the MCL or the allowable concentration based on a $25 \mathrm{mrem} / \mathrm{yr}$ performance objective (Table 4.3-1).

${ }^{c}$ Peak concentration is per Ci disposed of in ten trenches.

d Calculated by dividing the "Concentration limit" by the "Peak groundwater concentration", and dividing by 2 to normalize to one set of 5 trenches. For radionuclides with daughters, the lower limit calculated by this method for all radionuclides in the decay chain is the one reported as the inventory limit. Limiting radionuclide is printed in bold type.

e Inventory limit is based on radioactive daughter(s) only; parent was screened from consideration (Sect. 4.1).

${ }^{\mathrm{f}}$ Calculated by multiplying the concentration of the same nuclide as a parent by the ratio of the half-lives of the parent to the daughter in the current chain

${ }^{\mathrm{g}}$ Inventory is lowest inventory generating maximum fraction. Maximum fraction as part of sum-of-fractions for solubility-limited radionuclide - calculated by dividing peak concentration by MCL 
Table 6.2-1 Parameters Used in Screening Radionuclide Inventories for E-Area Low Level Waste Facility Disposal Units, and Lists of Radionuclides for Further Analysis.

\begin{tabular}{|c|c|c|}
\hline $\begin{array}{l}\text { Type of } \\
\text { Disposal Unit }\end{array}$ & $\begin{array}{c}\text { Volume of Waste } \\
\text { in Single Disposal } \\
\text { Unit }\left(\mathrm{m}^{3}\right)\end{array}$ & $\begin{array}{l}\text { Radionuclides Identified as Potentially Significant Contributors } \\
\text { to Dose for Inadvertent Intruders }\end{array}$ \\
\hline LAW Vaults & 48,000 & $\begin{array}{l}{ }^{3} \mathrm{H},{ }^{14} \mathrm{C},{ }^{59} \mathrm{Ni},{ }^{60} \mathrm{Co},{ }^{63} \mathrm{Ni},{ }^{79} \mathrm{Se},{ }^{87} \mathrm{Rb},{ }^{90} \mathrm{Sr}+\mathrm{d},{ }^{93} \mathrm{Zr}+\mathrm{d},{ }^{94} \mathrm{Nb},{ }^{99} \mathrm{Tc}, \\
{ }^{107} \mathrm{Pd},{ }^{126} \mathrm{Sn}+\mathrm{d},{ }^{132} \mathrm{I},{ }^{135} \mathrm{Cs},{ }^{137} \mathrm{Cs}+\mathrm{d},{ }^{151} \mathrm{Sm},{ }^{152} \mathrm{Eu},{ }^{154} \mathrm{Eu},{ }^{23} \mathrm{Th}+\mathrm{d}, \\
{ }^{232} \mathrm{U}+\mathrm{d},{ }^{233} \mathrm{U}+\mathrm{d},{ }^{234} \mathrm{U}+\mathrm{d},{ }^{235} \mathrm{U}+\mathrm{d},{ }^{236} \mathrm{U},{ }^{237} \mathrm{~Np},{ }^{238} \mathrm{U}+\mathrm{d},{ }^{238} \mathrm{Pu}+\mathrm{d}, \\
{ }^{239} \mathrm{Pu}+\mathrm{d},{ }^{240} \mathrm{Pu}+\mathrm{d},{ }^{241} \mathrm{Pu}+\mathrm{d},{ }^{242} \mathrm{Pu}+\mathrm{d},{ }^{244} \mathrm{Pu},{ }^{241} \mathrm{Am}+\mathrm{d},{ }^{243} \mathrm{Am}+\mathrm{d}, \\
{ }^{244} \mathrm{Cm}+\mathrm{d},{ }^{245} \mathrm{Cm}+\mathrm{d},{ }^{246} \mathrm{Cm},{ }^{247} \mathrm{Cm}+\mathrm{d},{ }^{248} \mathrm{Cm}+\mathrm{d},{ }^{249} \mathrm{Cf}+\mathrm{d},{ }^{250} \mathrm{Cf}+\mathrm{d}, \\
{ }^{251} \mathrm{Cf},{ }^{252} \mathrm{Cf}+\mathrm{d}\end{array}$ \\
\hline IL Vaults & 5700 & $\begin{array}{l}{ }^{3} \mathrm{H},{ }^{14} \mathrm{C},{ }^{59} \mathrm{Ni},{ }^{60} \mathrm{Co},{ }^{63} \mathrm{Ni},{ }^{79} \mathrm{Se},{ }^{90} \mathrm{Sr}+\mathrm{d},{ }^{93} \mathrm{Zr}+\mathrm{d},{ }^{99} \mathrm{Tc},{ }^{107} \mathrm{Pd},{ }^{126} \mathrm{Sn}+\mathrm{d}, \\
{ }^{129} \mathrm{I},{ }^{135} \mathrm{Cs},{ }^{137} \mathrm{Cs}+\mathrm{d},{ }^{154} \mathrm{Eu},{ }^{232} \mathrm{Th}+\mathrm{d},{ }^{23} \mathrm{U}+\mathrm{d},{ }^{233} \mathrm{U}+\mathrm{d},{ }^{234} \mathrm{U}+\mathrm{d}, \\
{ }^{235} \mathrm{U}+\mathrm{d},{ }^{236} \mathrm{U},{ }^{237} \mathrm{~Np},{ }^{238} \mathrm{U}+\mathrm{d},{ }^{238} \mathrm{Pu}+\mathrm{d},{ }^{239} \mathrm{Pu}+\mathrm{d},{ }^{240} \mathrm{Pu}+\mathrm{d},{ }^{241} \mathrm{Pu}+\mathrm{d}, \\
{ }^{242} \mathrm{Pu}+\mathrm{d},{ }^{244} \mathrm{Pu},{ }^{241} \mathrm{Am}+\mathrm{d},{ }^{242 \mathrm{~m}} \mathrm{Am}+\mathrm{d},{ }^{243} \mathrm{Am}+\mathrm{d},{ }^{244} \mathrm{Cm}+\mathrm{d},{ }^{245} \mathrm{Cm}+\mathrm{d}, \\
{ }^{246} \mathrm{Cm},{ }^{247} \mathrm{Cm}+\mathrm{d},{ }^{248} \mathrm{Cm}+\mathrm{d},{ }^{249} \mathrm{Cf}+\mathrm{d},{ }^{251} \mathrm{Cf},{ }^{252} \mathrm{Cf}+\mathrm{d}\end{array}$ \\
\hline Slit Trenches & 5760 & $\begin{array}{l}{ }^{3} \mathrm{H},{ }^{14} \mathrm{C},{ }^{59} \mathrm{Ni},{ }^{60} \mathrm{Co},{ }^{63} \mathrm{Ni},{ }^{79} \mathrm{Se},{ }^{87} \mathrm{Rb},{ }^{90} \mathrm{Sr}+\mathrm{d},{ }^{93} \mathrm{Zr}+\mathrm{d},{ }^{94} \mathrm{Nb},{ }^{99} \mathrm{Tc}, \\
{ }^{107} \mathrm{Pd},{ }^{113 \mathrm{~m}} \mathrm{Cd},{ }^{121 \mathrm{~m}} \mathrm{Sn},{ }^{126} \mathrm{Sn}+\mathrm{d},{ }^{129} \mathrm{I},{ }^{135} \mathrm{Cs},{ }^{137} \mathrm{Cs}+\mathrm{d},{ }^{151} \mathrm{Sm},{ }^{154} \mathrm{Eu}, \\
{ }^{228} \mathrm{Th},{ }^{232} \mathrm{Th}+\mathrm{d},{ }^{232} \mathrm{U}+\mathrm{d},{ }^{233} \mathrm{U}+\mathrm{d},{ }^{234} \mathrm{U}+\mathrm{d},{ }^{235} \mathrm{U}+\mathrm{d},{ }^{236} \mathrm{U},{ }^{237} \mathrm{~Np}, \\
{ }^{238} \mathrm{U}+\mathrm{d},{ }^{238} \mathrm{Pu}+\mathrm{d},{ }^{239} \mathrm{Pu}+\mathrm{d},{ }^{240} \mathrm{Pu}+\mathrm{d},{ }^{241} \mathrm{Pu}+\mathrm{d},{ }^{242} \mathrm{Pu}+\mathrm{d},{ }^{244} \mathrm{Pu}, \\
{ }^{241} \mathrm{Am}+\mathrm{d},{ }^{242 \mathrm{~m}} \mathrm{Am},{ }^{243} \mathrm{Am}+\mathrm{d},{ }^{242} \mathrm{Cm}+\mathrm{d},{ }^{243} \mathrm{Cm},{ }^{244} \mathrm{Cm}+\mathrm{d},{ }^{245} \mathrm{Cm}+\mathrm{d}, \\
{ }^{246} \mathrm{Cm},{ }^{247} \mathrm{Cm}+\mathrm{d},{ }^{248} \mathrm{Cm}+\mathrm{d},{ }^{24} \mathrm{Bk}+\mathrm{d},{ }^{249} \mathrm{Cf}+\mathrm{d},{ }^{250} \mathrm{Cf}+\mathrm{d},{ }^{251} \mathrm{Cf}, \\
{ }^{252} \mathrm{Cf}+\mathrm{d}\end{array}$ \\
\hline $\begin{array}{l}\text { Intimately- } \\
\text { mixed } \\
\text { Cement- } \\
\text { stabilized } \\
\text { Waste Units }\end{array}$ & 5760 & $\begin{array}{l}{ }^{3} \mathrm{H},{ }^{14} \mathrm{C},{ }^{59} \mathrm{Ni},{ }^{60} \mathrm{Co},{ }^{63} \mathrm{Ni},{ }^{79} \mathrm{Se},{ }^{87} \mathrm{Rb},{ }^{90} \mathrm{Sr}+\mathrm{d},{ }^{93} \mathrm{Zr}+\mathrm{d},{ }^{94} \mathrm{Nb},{ }^{99} \mathrm{Tc}, \\
{ }^{107} \mathrm{Pd},{ }^{126} \mathrm{Sn}+\mathrm{d},{ }^{129} \mathrm{I},{ }^{135} \mathrm{Cs},{ }^{137} \mathrm{Cs}+\mathrm{d},{ }^{151} \mathrm{Sm},{ }^{152} \mathrm{Eu},{ }^{154} \mathrm{Eu},{ }^{232} \mathrm{Th}+\mathrm{d}, \\
{ }^{232} \mathrm{U}+\mathrm{d},{ }^{233} \mathrm{U}+\mathrm{d},{ }^{234} \mathrm{U}+\mathrm{d},{ }^{235} \mathrm{U}+\mathrm{d},{ }^{236} \mathrm{U},{ }^{237} \mathrm{~Np},{ }^{238} \mathrm{U}+\mathrm{d},{ }^{238} \mathrm{Pu}+\mathrm{d}, \\
{ }^{239} \mathrm{Pu}+\mathrm{d},{ }^{240} \mathrm{Pu}+\mathrm{d},{ }^{241} \mathrm{Pu}+\mathrm{d},{ }^{242} \mathrm{Pu}+\mathrm{d},{ }^{244} \mathrm{Pu},{ }^{241} \mathrm{Am}+\mathrm{d},{ }^{243} \mathrm{Am}+\mathrm{d}, \\
{ }^{244} \mathrm{Cm}+\mathrm{d},{ }^{245} \mathrm{Cm}+\mathrm{d},{ }^{246} \mathrm{Cm},{ }^{247} \mathrm{Cm}+\mathrm{d},{ }^{248} \mathrm{Cm}+\mathrm{d},{ }^{249} \mathrm{Cf}+\mathrm{d},{ }^{250} \mathrm{Cf}+\mathrm{d}, \\
{ }^{251} \mathrm{Cf},{ }^{252} \mathrm{Cf}+\mathrm{d}\end{array}$ \\
\hline $\begin{array}{l}\text { Cement- } \\
\text { stabilized } \\
\text { Encapsulated } \\
\text { Waste Units }\end{array}$ & 5760 & $\begin{array}{l}{ }^{3} \mathrm{H},{ }^{14} \mathrm{C},{ }^{59} \mathrm{Ni},{ }^{60} \mathrm{Co},{ }^{63} \mathrm{Ni},{ }^{79} \mathrm{Se},{ }^{87} \mathrm{Rb},{ }^{90} \mathrm{Sr}+\mathrm{d},{ }^{93} \mathrm{Zr}+\mathrm{d},{ }^{94} \mathrm{Nb},{ }^{99} \mathrm{Tc}, \\
{ }^{107} \mathrm{Pd},{ }^{113 \mathrm{~m}} \mathrm{Cd},{ }^{121 \mathrm{~m}} \mathrm{Sn},{ }^{126} \mathrm{Sn}+\mathrm{d},{ }^{129} \mathrm{I},{ }^{135} \mathrm{Cs},{ }^{137} \mathrm{Cs}+\mathrm{d},{ }^{151} \mathrm{Sm},{ }^{154} \mathrm{Eu}, \\
{ }^{228} \mathrm{Th},{ }^{232} \mathrm{Th}+\mathrm{d},{ }^{232} \mathrm{U}+\mathrm{d},{ }^{233} \mathrm{U}+\mathrm{d},{ }^{234} \mathrm{U}+\mathrm{d},{ }^{235} \mathrm{U}+\mathrm{d},{ }^{236} \mathrm{U},{ }^{237} \mathrm{~Np}, \\
{ }^{238} \mathrm{U}+\mathrm{d},{ }^{238} \mathrm{Pu}+\mathrm{d},{ }^{239} \mathrm{Pu}+\mathrm{d},{ }^{240} \mathrm{Pu}+\mathrm{d},{ }^{241} \mathrm{Pu}+\mathrm{d},{ }^{242} \mathrm{Pu}+\mathrm{d},{ }^{244} \mathrm{Pu}, \\
{ }^{241} \mathrm{Am}+\mathrm{d},{ }^{242 \mathrm{~m}} \mathrm{Am},{ }^{243} \mathrm{Am}+\mathrm{d},{ }^{242} \mathrm{Cm}+\mathrm{d},{ }^{243} \mathrm{Cm},{ }^{244} \mathrm{Cm}+\mathrm{d},{ }^{245} \mathrm{Cm}+\mathrm{d}, \\
{ }^{246} \mathrm{Cm},{ }^{247} \mathrm{Cm}+\mathrm{d},{ }^{248} \mathrm{Cm}+\mathrm{d},{ }^{24} \mathrm{Bk}+\mathrm{d},{ }^{249} \mathrm{Cf}+\mathrm{d},{ }^{250} \mathrm{Cf}+\mathrm{d},{ }^{251} \mathrm{Cf}, \\
{ }^{252} \mathrm{Cf}+\mathrm{d}\end{array}$ \\
\hline $\begin{array}{l}\text { Naval Reactor } \\
\text { Components }\end{array}$ & 10000 & $\begin{array}{l}{ }^{3} \mathrm{H},{ }^{14} \mathrm{C},{ }^{59} \mathrm{Ni},{ }^{60} \mathrm{Co},{ }^{63} \mathrm{Ni},{ }^{79} \mathrm{Se},{ }^{90} \mathrm{Sr}+\mathrm{d},{ }^{93} \mathrm{Mo},{ }^{93} \mathrm{Zr}+\mathrm{d},{ }^{94} \mathrm{Nb},{ }^{99} \mathrm{Tc}, \\
{ }^{107} \mathrm{Pd},{ }^{126} \mathrm{Sn}+\mathrm{d},{ }^{129} \mathrm{I},{ }^{135} \mathrm{Cs},{ }^{137} \mathrm{Cs}+\mathrm{d},{ }^{151} \mathrm{Sm},{ }^{154} \mathrm{Eu},{ }^{232} \mathrm{Th}+\mathrm{d},{ }^{232} \mathrm{U}+\mathrm{d}, \\
{ }^{234} \mathrm{U}+\mathrm{d},{ }^{235} \mathrm{U}+\mathrm{d},{ }^{236} \mathrm{U},{ }^{237} \mathrm{~Np},{ }^{238} \mathrm{U}+\mathrm{d},{ }^{238} \mathrm{Pu}+\mathrm{d},{ }^{239} \mathrm{Pu}+\mathrm{d},{ }^{240} \mathrm{Pu}+\mathrm{d}, \\
{ }^{241} \mathrm{Pu}+\mathrm{d},{ }^{242} \mathrm{Pu}+\mathrm{d},{ }^{244} \mathrm{Pu},{ }^{241} \mathrm{Am}+\mathrm{d},{ }^{242 \mathrm{~m}} \mathrm{Am}+\mathrm{d},{ }^{243} \mathrm{Am}+\mathrm{d},{ }^{242} \mathrm{Cm}+\mathrm{d}, \\
{ }^{243} \mathrm{Cm},{ }^{244} \mathrm{Cm}+\mathrm{d},{ }^{245} \mathrm{Cm}+\mathrm{d},{ }^{246} \mathrm{Cm},{ }^{247} \mathrm{Cm}+\mathrm{d},{ }^{248} \mathrm{Cm}+\mathrm{d},{ }^{249} \mathrm{Bk}+\mathrm{d}, \\
{ }^{249} \mathrm{Cf}+\mathrm{d},{ }^{251} \mathrm{Cf}\end{array}$ \\
\hline
\end{tabular}

${ }^{a}$ Starting list of radionuclides (i.e., before screening) assumed to be the same as that for the LAW vaults.

b Assumes waste from 100 naval casks buried in an area $43 \mathrm{~m} \times 43 \mathrm{~m} \times 5.4 \mathrm{~m}$ deep. 
Table 6.3-10. Intruder-Based Radionuclide Disposal Limits for Ceme nt-Stabilized Encapsulated Waste Trenches - Agriculture Scenario at 700 Years

\begin{tabular}{|c|c|c|c|}
\hline Radionuclide $^{\mathrm{a}}$ & Fraction Remaining $^{\mathrm{b}}$ & $\begin{array}{c}\text { Concentration Limit } \\
\qquad\left(\mu \mathrm{Ci} / \mathrm{m}^{3}\right)\end{array}$ & $\begin{array}{l}\text { Intruder Limit }{ }^{\mathrm{d}} \\
(\mathrm{Ci} / 5 \text { trenches })\end{array}$ \\
\hline H-3 & $0.0 \mathrm{E}+00$ & $>1 \mathrm{E} 20$ & $>1 \mathrm{E} 20$ \\
\hline C- 14 & $7.4 \mathrm{E}-01$ & $1.5 \mathrm{E}+04$ & $4.3 \mathrm{E}+02$ \\
\hline C-14_KB & $8.2 \mathrm{E}-01$ & $1.3 \mathrm{E}+04$ & $3.9 \mathrm{E}+02$ \\
\hline Co- 60 & $1.1 \mathrm{E}-40^{\mathrm{e}}$ & $>1 \mathrm{E} 20$ & $>1 \mathrm{E} 20$ \\
\hline Ni-59 & 8.7E-01 & $2.8 \mathrm{E}+06$ & $8.1 \mathrm{E}+04$ \\
\hline $\mathrm{Ni}-63$ & $7.9 \mathrm{E}-03^{\mathrm{e}}$ & $1.2 \mathrm{E}+08$ & $3.3 \mathrm{E}+06$ \\
\hline $\mathrm{Se}-79$ & $1.1 \mathrm{E}-01$ & $1.3 \mathrm{E}+06$ & $3.7 E+04$ \\
\hline $\mathrm{Rb}-87$ & $5.7 \mathrm{E}-08$ & $1.5 \mathrm{E}+12$ & $4.5 \mathrm{E}+10$ \\
\hline Sr-90+d & $8.9 \mathrm{E}-19$ & $>1 \mathrm{E} 20$ & $2.8 \mathrm{E}+19$ \\
\hline $\mathrm{Zr}-93+\mathrm{d}$ & $9.7 \mathrm{E}-01$ & $4.2 E+06$ & $1.2 \mathrm{E}+05$ \\
\hline $\mathrm{Nb}-94$ & $9.8 \mathrm{E}-01^{\mathrm{e}}$ & $8.1 \mathrm{E}+01$ & $2.3 \mathrm{E}+00$ \\
\hline Мо-93 & $8.7 \mathrm{E}-01^{\mathrm{e}}$ & $2.2 \mathrm{E}+08$ & $6.3 \mathrm{E}+06$ \\
\hline Tc-99 & $1.1 \mathrm{E}-46$ & $>1 \mathrm{E} 20$ & $>1 \mathrm{E} 20$ \\
\hline Tc-99_KB & $7.9 \mathrm{E}-01$ & $1.9 \mathrm{E}+04$ & $5.6 \mathrm{E}+02$ \\
\hline $\mathrm{Pd}-10 \overline{7}$ & $1.9 \mathrm{E}-04$ & $2.7 \mathrm{E}+10$ & $7.8 \mathrm{E}+08$ \\
\hline Cd-113m & $3.7 \mathrm{E}-15^{\mathrm{e}}$ & $3.6 \mathrm{E}+17$ & $1.0 \mathrm{E}+16$ \\
\hline Sn-121m & $1.7 \mathrm{E}-03^{\mathrm{e}}$ & $2.1 \mathrm{E}+08$ & $6.0 \mathrm{E}+06$ \\
\hline Sn-126+d & $4.0 \mathrm{E}-01$ & $1.6 \mathrm{E}+02$ & $4.6 \mathrm{E}+00$ \\
\hline I- 129 & $9.9 \mathrm{E}-36$ & $>1 \mathrm{E} 20$ & $>1 \mathrm{E} 20$ \\
\hline I-129_KB & $9.6 \mathrm{E}-01$ & $2.1 \mathrm{E}+03$ & $6.0 \mathrm{E}+01$ \\
\hline I-129_XX & $1.0 \mathrm{E}+00$ & $2.0 \mathrm{E}+03$ & $5.7 \mathrm{E}+01$ \\
\hline Cs-135 & $4.9 \mathrm{E}-01$ & $2.8 \mathrm{E}+05$ & $7.9 \mathrm{E}+03$ \\
\hline Cs $-137+d$ & $1.0 \mathrm{E}-07^{\mathrm{e}}$ & $2.1 \mathrm{E}+09$ & $6.0 \mathrm{E}+07$ \\
\hline Sm-151 & $4.6 \mathrm{E}-03^{\mathrm{e}}$ & $3.6 \mathrm{E}+09$ & $1.0 \mathrm{E}+08$ \\
\hline $\mathrm{Eu}-152$ & $1.9 \mathrm{E}-16^{\mathrm{e}}$ & $5.7 \mathrm{E}+17$ & $1.6 \mathrm{E}+16$ \\
\hline Eu-154 & $1.1 \mathrm{E}-24^{\mathrm{e}}$ & $>1 \mathrm{E} 20$ & $>1 \mathrm{E} 20$ \\
\hline Th-228 & $8.0 \mathrm{E}-01^{\mathrm{e}}$ & $1.6 \mathrm{E}+04$ & $4.6 \mathrm{E}+02$ \\
\hline Th-232+d & 9.9E-01 & $4.9 \mathrm{E}+01$ & $1.4 \mathrm{E}+00$ \\
\hline $\mathrm{U}-232+\mathrm{d}$ & $1.2 \mathrm{E}-03$ & $6.1 \mathrm{E}+04$ & $1.7 \mathrm{E}+03$ \\
\hline$U-233+d$ & $9.9 \mathrm{E}-01$ & $4.4 \mathrm{E}+03$ & $1.3 \mathrm{E}+02$ \\
\hline $\mathrm{U}-234+\mathrm{d}$ & $9.9 \mathrm{E}-01$ & $1.4 \mathrm{E}+04$ & $4.2 \mathrm{E}+02$ \\
\hline $\mathrm{U}-235+\mathrm{d}$ & $9.9 \mathrm{E}-01$ & $9.2 \mathrm{E}+02$ & $2.7 \mathrm{E}+01$ \\
\hline U-236 & $9.9 \mathrm{E}-01$ & $1.6 \mathrm{E}+04$ & $4.6 \mathrm{E}+02$ \\
\hline $\mathrm{U}-238+\mathrm{d}$ & $1.0 \mathrm{E}+00$ & $4.3 \mathrm{E}+03$ & $1.2 \mathrm{E}+02$ \\
\hline $\mathrm{Np}-237+\mathrm{d}$ & $9.8 \mathrm{E}-01$ & $3.5 \mathrm{E}+02$ & $9.9 \mathrm{E}+00$ \\
\hline $\mathrm{Pu}-238+\mathrm{d}$ & $4.0 \mathrm{E}-03$ & $1.2 \mathrm{E}+06$ & $3.4 \mathrm{E}+04$ \\
\hline $\mathrm{Pu}-239+\mathrm{d}$ & $9.8 \mathrm{E}-01$ & $4.4 \mathrm{E}+03$ & $1.3 \mathrm{E}+02$ \\
\hline $\mathrm{Pu}-240+\mathrm{d}$ & $9.3 \mathrm{E}-01$ & $4.6 \mathrm{E}+03$ & $1.3 \mathrm{E}+02$ \\
\hline $\mathrm{Pu}-241+\mathrm{d}$ & $2.3 \mathrm{E}-15^{\mathrm{e}}$ & $2.8 \mathrm{E}+05$ & $8.0 \mathrm{E}+03$ \\
\hline $\mathrm{Pu}-242+\mathrm{d}$ & $9.9 \mathrm{E}-01$ & $4.6 \mathrm{E}+03$ & $1.3 \mathrm{E}+02$ \\
\hline $\mathrm{Pu}-244+\mathrm{d}$ & $9.9 \mathrm{E}-01$ & $3.4 \mathrm{E}+02$ & $9.8 \mathrm{E}+00$ \\
\hline$A m-241+d$ & $3.3 \mathrm{E}-01^{\mathrm{e}}$ & $9.2 \mathrm{E}+03$ & $2.7 \mathrm{E}+02$ \\
\hline Am-242m & $4.1 \mathrm{E}-02^{\mathrm{e}}$ & $7.2 \mathrm{E}+04$ & $2.1 \mathrm{E}+03$ \\
\hline$A m-243+d$ & $9.3 \mathrm{E}-01$ & $7.3 \mathrm{E}+02$ & $2.1 \mathrm{E}+01$ \\
\hline $\mathrm{Cm}-242+\mathrm{d}$ & $0.0 \mathrm{E}+00^{\mathrm{e}}$ & $2.3 \mathrm{E}+08$ & $6.6 \mathrm{E}+06$ \\
\hline $\mathrm{Cm}-243$ & $4.1 \mathrm{E}-08^{\mathrm{e}}$ & $2.7 \mathrm{E}+10$ & $7.8 \mathrm{E}+08$ \\
\hline $\mathrm{Cm}-244+\mathrm{d}$ & $2.3 \mathrm{E}-12^{\mathrm{e}}$ & $1.7 \mathrm{E}+06$ & $4.8 \mathrm{E}+04$ \\
\hline $\mathrm{Cm}-245+\mathrm{d}$ & $9.4 \mathrm{E}-01$ & $1.2 \mathrm{E}+03$ & $3.3 \mathrm{E}+01$ \\
\hline $\mathrm{Cm}-246$ & $9.0 \mathrm{E}-01$ & $4.6 \mathrm{E}+03$ & $1.3 \mathrm{E}+02$ \\
\hline
\end{tabular}


Table 6.3-10. Intruder-Based Radionuclide Disposal Limits for Ceme nt-Stabilized Encapsulated Waste Trenches - Agriculture Scenario at 700 Years

\begin{tabular}{|c|c|c|c|}
\hline Radionuclide $^{\mathrm{a}}$ & Fraction Remaining $^{\mathrm{b}}$ & $\begin{array}{l}\text { Concentration Limit } \\
\left(\mu \mathrm{Ci} / \mathrm{m}^{3}\right)\end{array}$ & $\begin{array}{r}\text { Intruder Limit }{ }^{\mathrm{d}} \\
(\mathrm{Ci} / 5 \text { trenches })\end{array}$ \\
\hline $\mathrm{Cm}-247+\mathrm{d}$ & $1.0 \mathrm{E}+00$ & $3.7 \mathrm{E}+02$ & $1.1 \mathrm{E}+01$ \\
\hline $\mathrm{Cm}-248+\mathrm{d}$ & 9.9E-01 & $1.2 \mathrm{E}+03$ & $3.4 \mathrm{E}+01$ \\
\hline Bk-249 +d & $0.0 \mathrm{E}+00$ & $5.6 \mathrm{E}+05$ & $1.6 \mathrm{E}+04$ \\
\hline Cf- $249+d$ & $2.5 \mathrm{E}-01$ & $1.4 \mathrm{E}+03$ & 4.0E+01 \\
\hline Cf $-250+d$ & $7.8 \mathrm{E}-17^{\mathrm{e}}$ & $2.4 \mathrm{E}+07$ & $6.8 \mathrm{E}+05$ \\
\hline Cf-251 & $5.8 \mathrm{E}-01^{\mathrm{e}}$ & $1.8 \mathrm{E}+03$ & $5.2 \mathrm{E}+01$ \\
\hline Cf $-252+d$ & $3.9 \mathrm{E}-81^{\mathrm{e}}$ & $1.6 \mathrm{E}+08$ & $4.7 \mathrm{E}+06$ \\
\hline \multirow{4}{*}{\multicolumn{4}{|c|}{$\begin{array}{l}\text { a The notation "+d" indicates that daughters were incorporated into the EDE. } \\
\text { b Considers radioactive decay and leaching unless otherwise noted. } \\
\text { c Limit on average concentration in disposed waste; obtained from Eq. } 6.3-5 \\
\text { d Limit on inventory per } 5 \text { trenches; obtained from Eq. 6.3-4, assuming a volume of } 2.88 \mathrm{E} 4 \mathrm{~m}^{3} \text { for } \\
5 \text { trenches. }\end{array}$}} \\
\hline & & & \\
\hline & & & \\
\hline & & & \\
\hline \multicolumn{4}{|c|}{${ }^{\mathrm{e}}$ Only radioactive decay accounted for. } \\
\hline
\end{tabular}


Table 6.3-16. Intruder-Based Radionuclide Disposal Limits for CementStabilized Encapsulated Waste - Resident Scenario at 100 Years

\begin{tabular}{|c|c|c|c|}
\hline \multirow[b]{2}{*}{ Radionuclide $^{\mathrm{a}}$} & \multicolumn{3}{|c|}{ Concentration } \\
\hline & $\begin{array}{c}\text { Fraction } \\
\text { Remaining }\end{array}$ & $\begin{array}{l}\text { Limit }^{c} \\
\left(\mu \mathrm{Ci} / \mathrm{m}^{3}\right)\end{array}$ & $\begin{array}{l}\text { Inventory Limit } \\
(\mathrm{Ci} / 5 \text { trenches })\end{array}$ \\
\hline Co-60 & $2.0 \mathrm{E}-06^{\mathrm{e}}$ & $7.4 \mathrm{E}+10$ & $2.1 \mathrm{E}+09$ \\
\hline $\mathrm{Nb}-94$ & $1.0 \mathrm{E}+00^{\mathrm{e}}$ & $1.2 \mathrm{E}+06$ & $3.4 \mathrm{E}+04$ \\
\hline Sn-126+d & $1.0 \mathrm{E}+00$ & $2.0 \mathrm{E}+06$ & $5.7 \mathrm{E}+04$ \\
\hline Cs $-137+d$ & $1.0 \mathrm{E}-01^{\mathrm{e}}$ & $7.6 \mathrm{E}+07$ & $2.2 \mathrm{E}+06$ \\
\hline $\mathrm{Eu}-152$ & $5.7 \mathrm{E}-03^{\mathrm{e}}$ & $8.5 \mathrm{E}+07$ & $2.4 \mathrm{E}+06$ \\
\hline Eu-154 & $3.8 \mathrm{E}-04^{\mathrm{e}}$ & $1.3 \mathrm{E}+09$ & $3.6 \mathrm{E}+07$ \\
\hline $\mathrm{Th}-232+\mathrm{d}$ & $1.0 \mathrm{E}+00$ & $4.1 \mathrm{E}+04$ & $1.2 \mathrm{E}+03$ \\
\hline $\mathrm{U}-232+\mathrm{d}$ & $3.8 \mathrm{E}-01$ & $1.1 \mathrm{E}+05$ & $3.2 \mathrm{E}+03$ \\
\hline $\mathrm{U}-233+\mathrm{d}$ & $1.0 \mathrm{E}+00$ & $5.2 \mathrm{E}+08$ & $1.5 \mathrm{E}+07$ \\
\hline $\mathrm{U}-234+\mathrm{d}$ & $1.0 \mathrm{E}+00$ & $6.6 \mathrm{E}+09$ & $1.9 \mathrm{E}+08$ \\
\hline $\mathrm{U}-235+\mathrm{d}$ & $1.0 \mathrm{E}+00$ & $2.2 \mathrm{E}+10$ & $6.4 \mathrm{E}+08$ \\
\hline $\mathrm{U}-238+\mathrm{d}$ & $1.0 \mathrm{E}+00$ & $6.7 \mathrm{E}+07$ & $1.9 \mathrm{E}+06$ \\
\hline $\mathrm{Np}-237+\mathrm{d}$ & $1.0 \mathrm{E}+00$ & $6.0 \mathrm{E}+08$ & $1.7 \mathrm{E}+07$ \\
\hline $\mathrm{Pu}-241+\mathrm{d}$ & $8.1 \mathrm{E}-03^{\mathrm{e}}$ & $9.0 \mathrm{E}+13$ & $2.6 \mathrm{E}+12$ \\
\hline $\mathrm{Pu}-244+\mathrm{d}$ & $1.0 \mathrm{E}+00$ & $3.4 \mathrm{E}+06$ & $9.8 \mathrm{E}+04$ \\
\hline$A m-241+d$ & $8.5 \mathrm{E}-01$ & $3.0 \mathrm{E}+12$ & $8.6 \mathrm{E}+10$ \\
\hline $\mathrm{Am}-243+\mathrm{d}$ & $9.9 \mathrm{E}-01$ & $4.0 \mathrm{E}+09$ & $1.2 \mathrm{E}+08$ \\
\hline $\mathrm{Cm}-243$ & $8.8 \mathrm{E}-02^{\mathrm{e}}$ & $9.3 \mathrm{E}+10$ & $2.7 \mathrm{E}+09$ \\
\hline $\mathrm{Cm}-245+\mathrm{d}$ & $9.9 \mathrm{E}-01$ & $3.6 \mathrm{E}+14$ & $1.0 \mathrm{E}+13$ \\
\hline $\mathrm{Cm}-247+\mathrm{d}$ & $1.0 \mathrm{E}+00$ & $1.3 \mathrm{E}+08$ & $3.8 \mathrm{E}+06$ \\
\hline $\mathrm{Cm}-248+\mathrm{d}$ & $1.0 \mathrm{E}+00$ & $4.8 \mathrm{E}+12$ & $1.4 \mathrm{E}+11$ \\
\hline Bk-249 + d & $6.3 \mathrm{E}-35$ & $7.7 \mathrm{E}+10$ & $2.2 \mathrm{E}+09$ \\
\hline Cf-249+d & $8.2 \mathrm{E}-01$ & $1.9 \mathrm{E}+08$ & $5.6 \mathrm{E}+06$ \\
\hline
\end{tabular}


Table 6.3-20. Intruder-Based Radionuclide Disposal Limits for CementStabilized Encapsulated Waste - Post-Drilling Scenario at 300 Years

\begin{tabular}{|c|c|c|c|}
\hline Radionuclide $^{\mathrm{a}}$ & $\begin{array}{c}\text { Fraction } \\
\text { Remaining }\end{array}$ & $\begin{array}{l}\text { Concentration } \\
\text { Limit }^{c} \\
(\mu \mathrm{Ci} / \mathrm{cu} \mathrm{m})\end{array}$ & $\begin{array}{l}\text { Inventory Limit } \\
\text { (Ci/5 trenches })\end{array}$ \\
\hline H-3 & $3.3 \mathrm{E}-10$ & $7.9 \mathrm{E}+14$ & $2.3 E+13$ \\
\hline C-14 & $9.6 \mathrm{E}-01$ & $6.9 \mathrm{E}+04$ & $2.0 \mathrm{E}+03$ \\
\hline C-14_KB & $9.6 \mathrm{E}-01$ & $6.9 \mathrm{E}+04$ & $2.0 \mathrm{E}+03$ \\
\hline Co- 60 & $7.4 \mathrm{E}-18^{\mathrm{e}}$ & $>1 \mathrm{E} 20$ & $>1 \mathrm{E} 20$ \\
\hline $\mathrm{Ni}-59$ & $1.0 \mathrm{E}+00$ & $1.5 \mathrm{E}+07$ & $4.3 \mathrm{E}+05$ \\
\hline $\mathrm{Ni}-63$ & $1.3 \mathrm{E}-01^{\mathrm{e}}$ & $4.4 \mathrm{E}+07$ & $1.3 \mathrm{E}+06$ \\
\hline Se-79 & $9.9 \mathrm{E}-01$ & $8.3 \mathrm{E}+05$ & $2.4 \mathrm{E}+04$ \\
\hline $\mathrm{Rb}-87$ & $1.0 \mathrm{E}+00$ & $5.3 \mathrm{E}+05$ & $1.5 \mathrm{E}+04$ \\
\hline Sr- $90+d$ & $6.3 \mathrm{E}-04$ & $8.6 \mathrm{E}+06$ & $2.5 \mathrm{E}+05$ \\
\hline $\mathrm{Zr}-93+\mathrm{d}$ & $1.0 \mathrm{E}+00$ & $3.2 \mathrm{E}+07$ & $9.2 \mathrm{E}+05$ \\
\hline $\mathrm{Nb}-94$ & $9.9 \mathrm{E}-01^{\mathrm{e}}$ & $9.4 \mathrm{E}+04$ & $2.7 \mathrm{E}+03$ \\
\hline Мо-93 & $9.4 \mathrm{E}-01^{\mathrm{e}}$ & $1.4 \mathrm{E}+10$ & $4.0 \mathrm{E}+08$ \\
\hline Tc-99 & $5.7 \mathrm{E}-01$ & $1.6 \mathrm{E}+05$ & $4.6 \mathrm{E}+03$ \\
\hline Tc-99_KB & $1.0 \mathrm{E}+00$ & $9.2 \mathrm{E}+04$ & $2.7 \mathrm{E}+03$ \\
\hline Pd-107 & $1.0 \mathrm{E}+00$ & $3.2 \mathrm{E}+07$ & $9.2 \mathrm{E}+05$ \\
\hline Cd-113m & $6.5 \mathrm{E}-07^{\mathrm{e}}$ & $1.2 \mathrm{E}+10$ & $3.5 \mathrm{E}+08$ \\
\hline $\mathrm{Sn}-121 \mathrm{~m}$ & $6.5 \mathrm{E}-02^{\mathrm{e}}$ & $2.9 \mathrm{E}+08$ & $8.4 \mathrm{E}+06$ \\
\hline Sn-126+d & $1.0 \mathrm{E}+00$ & $7.1 \mathrm{E}+04$ & $2.0 \mathrm{E}+03$ \\
\hline I- 129 & 7.9E-01 & $1.6 \mathrm{E}+04$ & $4.5 \mathrm{E}+02$ \\
\hline I-129_KB & $1.0 \mathrm{E}+00$ & $1.2 \mathrm{E}+04$ & $3.6 \mathrm{E}+02$ \\
\hline Cs -135 & $1.0 \mathrm{E}+00$ & $8.2 \mathrm{E}+05$ & $2.4 \mathrm{E}+04$ \\
\hline Cs $-137+d$ & $1.0 \mathrm{E}-03^{\mathrm{e}}$ & $7.9 \mathrm{E}+07$ & $2.3 E+06$ \\
\hline $\mathrm{Sm}-151$ & $9.9 \mathrm{E}-02^{\mathrm{e}}$ & $1.1 \mathrm{E}+09$ & $3.1 \mathrm{E}+07$ \\
\hline Eu-152 & $1.8 \mathrm{E}-07^{\mathrm{e}}$ & $7.0 \mathrm{E}+11$ & $2.0 \mathrm{E}+10$ \\
\hline Eu-154 & $5.5 \mathrm{E}-11^{\mathrm{e}}$ & $2.2 \mathrm{E}+15$ & $6.2 E+13$ \\
\hline Th-232+d & $1.0 \mathrm{E}+00$ & $1.7 \mathrm{E}+04$ & $4.8 \mathrm{E}+02$ \\
\hline $\mathrm{U}-232+\mathrm{d}$ & $5.5 \mathrm{E}-02$ & $3.2 \mathrm{E}+05$ & $9.3 \mathrm{E}+03$ \\
\hline $\mathrm{U}-233+\mathrm{d}$ & $1.0 \mathrm{E}+00$ & $1.2 \mathrm{E}+05$ & $3.5 E+03$ \\
\hline $\mathrm{U}-234+\mathrm{d}$ & $1.0 \mathrm{E}+00$ & $1.4 \mathrm{E}+05$ & $4.0 \mathrm{E}+03$ \\
\hline $\mathrm{U}-235+\mathrm{d}$ & $1.0 \mathrm{E}+00$ & $1.1 \mathrm{E}+05$ & $3.2 \mathrm{E}+03$ \\
\hline U-236 & $1.0 \mathrm{E}+00$ & $1.4 \mathrm{E}+05$ & $4.2 \mathrm{E}+03$ \\
\hline $\mathrm{U}-238+\mathrm{d}$ & $1.0 \mathrm{E}+00$ & $1.5 \mathrm{E}+05$ & $4.4 \mathrm{E}+03$ \\
\hline $\mathrm{Np}-237+\mathrm{d}$ & $1.0 \mathrm{E}+00$ & $4.2 \mathrm{E}+03$ & $1.2 \mathrm{E}+02$ \\
\hline $\mathrm{Pu}-238+\mathrm{d}$ & $9.3 \mathrm{E}-02$ & $5.0 \mathrm{E}+05$ & $1.4 \mathrm{E}+04$ \\
\hline$P u-239+d$ & 9.9E-01 & $4.1 \mathrm{E}+04$ & $1.2 \mathrm{E}+03$ \\
\hline $\mathrm{Pu}-240+\mathrm{d}$ & $9.7 \mathrm{E}-01$ & $4.2 \mathrm{E}+04$ & $1.2 \mathrm{E}+03$ \\
\hline $\mathrm{Pu}-241+\mathrm{d}$ & $5.4 \mathrm{E}-07$ & $1.6 \mathrm{E}+06$ & $4.5 \mathrm{E}+04$ \\
\hline $\mathrm{Pu}-242+\mathrm{d}$ & $1.0 \mathrm{E}+00$ & $4.3 \mathrm{E}+04$ & $1.2 \mathrm{E}+03$ \\
\hline $\mathrm{Pu}-244+\mathrm{d}$ & $1.0 \mathrm{E}+00$ & $4.0 \mathrm{E}+04$ & $1.2 \mathrm{E}+03$ \\
\hline$A m-241+d$ & $6.2 \mathrm{E}-01^{\mathrm{e}}$ & $5.2 \mathrm{E}+04$ & $1.5 \mathrm{E}+03$ \\
\hline Am-242m & $2.5 \mathrm{E}-01^{\mathrm{e}}$ & $1.3 \mathrm{E}+05$ & $3.8 \mathrm{E}+03$ \\
\hline$A m-243+d$ & $9.7 \mathrm{E}-01$ & $3.2 \mathrm{E}+04$ & $9.2 \mathrm{E}+02$ \\
\hline $\mathrm{Cm}-242+\mathrm{d}$ & $0.0 \mathrm{E}+00^{\mathrm{e}}$ & $9.7 \mathrm{E}+07$ & $2.8 \mathrm{E}+06$ \\
\hline $\mathrm{Cm}-243$ & $6.8 \mathrm{E}-04^{\mathrm{e}}$ & $8.8 \mathrm{E}+07$ & $2.5 \mathrm{E}+06$ \\
\hline $\mathrm{Cm}-244+\mathrm{d}$ & $1.0 \mathrm{E}-05^{\mathrm{e}}$ & $1.5 \mathrm{E}+07$ & $4.4 \mathrm{E}+05$ \\
\hline $\mathrm{Cm}-245+\mathrm{d}$ & $9.8 \mathrm{E}-01$ & $2.8 \mathrm{E}+04$ & $8.0 \mathrm{E}+02$ \\
\hline $\mathrm{Cm}-246$ & $9.6 \mathrm{E}-01$ & $4.2 \mathrm{E}+04$ & $1.2 \mathrm{E}+03$ \\
\hline $\mathrm{Cm}-247+\mathrm{d}$ & $1.0 \mathrm{E}+00$ & $3.9 \mathrm{E}+04$ & $1.1 \mathrm{E}+03$ \\
\hline $\mathrm{Cm}-248+\mathrm{d}$ & $1.0 \mathrm{E}+00$ & $1.1 \mathrm{E}+04$ & $3.3 \mathrm{E}+02$ \\
\hline
\end{tabular}


Table 6.3-20. Intruder-Based Radionuclide Disposal Limits for CementStabilized Encapsulated Waste - Post-Drilling Scenario at 300 Years

\begin{tabular}{lccc} 
Radionuclide $^{\mathrm{a}}$ & $\begin{array}{c}\text { Fraction } \\
\text { Remaining }^{\mathrm{b}}\end{array}$ & $\begin{array}{c}\text { Concentration } \\
\text { Limit }^{\mathrm{c}} \\
(\mu \mathrm{Ci} / \mathrm{cu} \mathrm{m})\end{array}$ & $\begin{array}{c}\text { Inventory Limit }^{\mathrm{d}} \\
(\mathrm{Ci} / 5 \text { trenches })\end{array}$ \\
\hline $\mathrm{Bk}-249+\mathrm{d}$ & $0.0 \mathrm{E}+00$ & $2.5 \mathrm{E}+07$ & $7.3 \mathrm{E}+05$ \\
$\mathrm{Cf}-249+\mathrm{d}$ & $5.5 \mathrm{E}-01$ & $6.3 \mathrm{E}+04$ & $1.8 \mathrm{E}+03$ \\
$\mathrm{Cf}-250+\mathrm{d}$ & $1.3 \mathrm{E}-07^{\mathrm{e}}$ & $5.3 \mathrm{E}+08$ & $1.5 \mathrm{E}+07$ \\
$\mathrm{Cf}-251$ & $7.9 \mathrm{E}-01^{\mathrm{e}}$ & $4.8 \mathrm{E}+04$ & $1.4 \mathrm{E}+03$ \\
$\mathrm{Cf}-252+\mathrm{d}$ & $0.0 \mathrm{E}+00$ & $1.6 \mathrm{E}+09$ & $4.5 \mathrm{E}+07$ \\
\hline
\end{tabular}

a The notation "+d" indicates that daughters were incorporated into the EDE.

b Considers radioactive decay and leaching unless otherwise noted.

c Limit on average concentration in disposed waste; obtained from Eq. 6.3-5

d Limit on inventory per 5 trenches; obtained from Eq. 6.3-4, assuming a volume of 28,800 $\mathrm{m}^{3}$ for 5 trenches.

e Only radioactive decay accounted for. 
Table 7.1-6. Inventory Limits for Cement-Stabilized Encapsulated Waste Trenches and Limiting Pathway

\begin{tabular}{|c|c|c|}
\hline Radionuclide $^{\mathrm{a}}$ & $\begin{array}{l}\text { Inventory limit } \\
\mathrm{Ci} / 5 \text { trenches }\end{array}$ & Limiting pathway \\
\hline H-3 & $4.1 \mathrm{E}+05$ & air \\
\hline C-14 & $5.7 \mathrm{E}+01$ & gw \\
\hline C-14_KB & $6.2 \mathrm{E}+01$ & gw \\
\hline $\mathrm{Co}-60$ & $2.1 \mathrm{E}+09$ & resident \\
\hline $\mathrm{Ni}-59$ & $9.3 \mathrm{E}+02$ & gw \\
\hline $\mathrm{Ni}-63$ & $1.3 \mathrm{E}+06$ & post-drilling \\
\hline Se-79 & $9.3 \mathrm{E}+01$ & gw \\
\hline $\mathrm{Rb}-87$ & $1.4 \mathrm{E}+01$ & gw \\
\hline $\mathrm{Sr}-90+\mathrm{d}$ & $1.6 \mathrm{E}+05$ & gw \\
\hline $\mathrm{Zr}-93+\mathrm{d}$ & $4.0 \mathrm{E}+04$ & gw \\
\hline $\mathrm{Nb}-94$ & $2.3 \mathrm{E}+00$ & agriculture \\
\hline Мо-93 & $6.3 E+06$ & agriculture \\
\hline Tc-99 & $3.8 \mathrm{E}-01$ & gw \\
\hline Tc-99_KB & $2.1 \mathrm{E}+01$ & gw \\
\hline $\mathrm{Pd}-10 \overline{7}$ & $1.8 \mathrm{E}+03$ & gw \\
\hline Cd-113m & $3.5 \mathrm{E}+08$ & post-drilling \\
\hline $\mathrm{Sn}-121 \mathrm{~m}$ & $6.0 \mathrm{E}+06$ & agriculture \\
\hline Sn-126+d & $4.6 \mathrm{E}+00$ & agriculture \\
\hline I- 129 & $6.1 \mathrm{E}-04$ & gw \\
\hline I- $129 \mathrm{~KB}$ & $1.1 \mathrm{E}-01$ & gw \\
\hline I-129_A & $9.2 \mathrm{E}-03$ & gw \\
\hline I-129_B & $2.5 \mathrm{E}-02$ & gw \\
\hline I-129_C & $1.7 \mathrm{E}-02$ & gw \\
\hline I-129_D & $8.0 \mathrm{E}-02$ & gw \\
\hline I-129_E & $5.7 \mathrm{E}-02$ & gw \\
\hline I-129_F & $1.8 \mathrm{E}-02$ & gw \\
\hline I-129_G & $6.2 \mathrm{E}-04$ & gw \\
\hline I-129_H & $2.9 \mathrm{E}-03$ & gw \\
\hline I-129_I & $8.9 \mathrm{E}-02$ & gw \\
\hline I-129_J & $7.4 \mathrm{E}-04$ & gw \\
\hline I-129_S & $1.7 \mathrm{E}-01$ & gw \\
\hline I-129_10K & 2.9E-01 & gw \\
\hline Cs $-13 \overline{5}$ & $5.1 \mathrm{E}+02$ & gw \\
\hline Cs $-137+d$ & $2.2 \mathrm{E}+06$ & resident \\
\hline Sm-151 & $3.1 \mathrm{E}+07$ & post-drilling \\
\hline Eu-152 & $2.4 \mathrm{E}+06$ & resident \\
\hline Eu-154 & $3.6 \mathrm{E}+07$ & resident \\
\hline Th-228 & $4.6 \mathrm{E}+02$ & agriculture \\
\hline Th-232+d & $1.4 \mathrm{E}+00$ & agriculture \\
\hline $\mathrm{U}-232+\mathrm{d}$ & $1.7 \mathrm{E}+03$ & agriculture \\
\hline $\mathrm{U}-233+\mathrm{d}$ & $2.3 \mathrm{E}+01$ & gw \\
\hline $\mathrm{U}-234+\mathrm{d}$ & $2.3 \mathrm{E}+01$ & gw \\
\hline $\mathrm{U}-235+\mathrm{d}$ & $1.1 \mathrm{E}+01$ & gw \\
\hline U-236 & $2.4 \mathrm{E}+01$ & gw \\
\hline $\mathrm{U}-238+\mathrm{d}$ & $1.2 \mathrm{E}+02$ & agriculture \\
\hline Np-237 & $3.7 \mathrm{E}-01$ & gw \\
\hline $\mathrm{Pu}-238+\mathrm{d}$ & $1.4 \mathrm{E}+04$ & post-drilling \\
\hline $\mathrm{Pu}-239+\mathrm{d}$ & $1.3 \mathrm{E}+02$ & agriculture \\
\hline $\mathrm{Pu}-240+\mathrm{d}$ & $2.7 \mathrm{E}+00$ & gw \\
\hline $\mathrm{Pu}-241+\mathrm{d}$ & $8.0 \mathrm{E}+03$ & agriculture \\
\hline
\end{tabular}


Table 7.1-6. Inventory Limits for Cement-Stabilized Encapsulated Waste Trenches and Limiting Pathway

\begin{tabular}{lcc}
\hline Radionuclide $^{\text {a }}$ & $\begin{array}{c}\text { Inventory limit } \\
\text { Ci/ } 5 \text { trenches }\end{array}$ & Limiting pathway \\
\hline $\mathrm{Pu}-242+\mathrm{d}$ & $1.0 \mathrm{E}+00$ & $\mathrm{gw}$ \\
$\mathrm{Pu}-244+\mathrm{d}$ & $1.1 \mathrm{E}+00$ & $\mathrm{gw}$ \\
$\mathrm{Am}-241+\mathrm{d}$ & $2.7 \mathrm{E}+02$ & agriculture \\
$\mathrm{Am}-242 \mathrm{~m}+\mathrm{d}$ & $2.1 \mathrm{E}+03$ & agriculture \\
$\mathrm{Am}-243+\mathrm{d}$ & $2.1 \mathrm{E}+01$ & agriculture \\
$\mathrm{Cm}-242+\mathrm{d}$ & $2.8 \mathrm{E}+06$ & post-drilling \\
$\mathrm{Cm}-243$ & $2.5 \mathrm{E}+06$ & post-drilling \\
$\mathrm{Cm}-244+\mathrm{d}$ & $4.8 \mathrm{E}+04$ & agriculture \\
$\mathrm{Cm}-245+\mathrm{d}$ & $3.3 \mathrm{E}+01$ & agriculture \\
$\mathrm{Cm}-246$ & $1.3 \mathrm{E}+02$ & agriculture \\
$\mathrm{Cm}-247+\mathrm{d}$ & $1.1 \mathrm{E}+01$ & agriculture \\
$\mathrm{Cm}-248+\mathrm{d}$ & $3.4 \mathrm{E}+01$ & agriculture \\
$\mathrm{Bk}-249+\mathrm{d}$ & $1.6 \mathrm{E}+04$ & agriculture \\
$\mathrm{Cf}-249+\mathrm{d}$ & $4.0 \mathrm{E}+01$ & agriculture \\
$\mathrm{Cf}-250+\mathrm{d}$ & $6.8 \mathrm{E}+05$ & agriculture \\
$\mathrm{Cf}-251$ & $5.2 \mathrm{E}+01$ & agriculture \\
$\mathrm{Cf}-252+\mathrm{d}$ & $4.7 \mathrm{E}+06$ & agriculture \\
\hline
\end{tabular}

Note: Values in this table are rounded to the appropriate number of significant digits using Microsoft Excel 97 SR-2 (h)

a " $+\mathrm{d}$ " indicates potentially-significant short- and long-lived daughters are accounted for in the limit. 


\begin{tabular}{|c|c|c|c|c|}
\hline \multicolumn{5}{|c|}{ Table A.6. Projected Inventory for Cement-stabilized Encapsulated Waste } \\
\hline $\begin{array}{l}\text { Radio- } \\
\text { Nuclide }\end{array}$ & $\begin{array}{l}\text { Single Used } \\
\text { Equipment } \\
\text { Storage Area }\end{array}$ & $\begin{array}{c}\text { Combined Use } \\
\text { Equipment } \\
\text { Storage Area } \\
\text { with } 55 \% \\
\text { Reduction }^{\mathrm{a}}\end{array}$ & Disposed & $\begin{array}{c}\text { Projected } \\
\mathrm{K} \text { and L Basin } \\
\text { Resins }\end{array}$ \\
\hline & $\begin{array}{c}\text { Actual } \\
\text { Inventory }\end{array}$ & Actual Inventory & Inventory & Inventory \\
\hline & $(\mathrm{Ci})$ & (Ci) & (Ci) & (Ci) \\
\hline $\begin{array}{l}\text { Other } \\
\text { Alpha }\end{array}$ & & & $4.95 \mathrm{E}-05$ & \\
\hline $\begin{array}{l}\text { Other } \\
\text { Beta/ } \\
\text { Gamma }\end{array}$ & & & $4.71 \mathrm{E}-04$ & \\
\hline Ag-110 & $3.39 \mathrm{E}-11$ & $2.98 \mathrm{E}-11$ & & \\
\hline Ag-110m & $2.05 \mathrm{E}+00$ & $1.80 \mathrm{E}+00$ & & \\
\hline Am-241 & $1.54 \mathrm{E}-02$ & $1.36 \mathrm{E}-02$ & $1.46 \mathrm{E}-02$ & $5.64 \mathrm{E}+00$ \\
\hline Am-243 & & & $7.48 \mathrm{E}-04$ & \\
\hline $\mathrm{Ba}-137 \mathrm{~m}$ & $8.39 \mathrm{E}+03$ & $7.38 \mathrm{E}+03$ & $1.90 \mathrm{E}+03$ & $3.33 \mathrm{E}+03$ \\
\hline Ba-140 & $3.39 \mathrm{E}-11$ & $2.98 \mathrm{E}-11$ & & \\
\hline $\mathrm{C}-14$ & & & $2.15 \mathrm{E}-02$ & \\
\hline C-14_KB & & & & $1.57 \mathrm{E}+02$ \\
\hline $\mathrm{Ce}-141$ & $3.39 \mathrm{E}-11$ & $2.98 \mathrm{E}-11$ & & \\
\hline Ce-144 & $1.14 \mathrm{E}+03$ & $1.00 \mathrm{E}+03$ & $5.66 \mathrm{E}-03$ & \\
\hline Cf-249 & & & $8.77 \mathrm{E}-05$ & \\
\hline Cf-251 & & & $8.78 \mathrm{E}-05$ & \\
\hline Cf-252 & & & $8.76 \mathrm{E}-05$ & \\
\hline Cm-243 & & & $1.47 \mathrm{E}-04$ & \\
\hline Cm-244 & & & $1.96 \mathrm{E}-01$ & \\
\hline $\mathrm{Cm}-245$ & & & $1.54 \mathrm{E}-05$ & \\
\hline $\mathrm{Cm}-246$ & & & $8.76 \mathrm{E}-05$ & \\
\hline $\mathrm{Cm}-247$ & & & $8.76 \mathrm{E}-05$ & \\
\hline Cm-248 & & & $8.76 \mathrm{E}-05$ & \\
\hline Co-60 & $1.11 \mathrm{E}+03$ & $9.77 \mathrm{E}+02$ & $6.82 \mathrm{E}-03$ & \\
\hline Cs-134 & $4.04 \mathrm{E}+03$ & $3.56 \mathrm{E}+03$ & $1.91 \mathrm{E}-05$ & \\
\hline Cs-135 & $2.61 \mathrm{E}-02$ & $2.30 \mathrm{E}-02$ & 4.34E-06 & \\
\hline Cs-137 & $8.39 \mathrm{E}+03$ & $7.38 \mathrm{E}+03$ & $2.01 \mathrm{E}+03$ & $3.52 \mathrm{E}+03$ \\
\hline Eu-152 & & & $4.24 \mathrm{E}-06$ & \\
\hline Eu-154 & $4.24 \mathrm{E}+02$ & $3.73 \mathrm{E}+02$ & $4.85 \mathrm{E}-04$ & \\
\hline Eu-155 & $2.48 \mathrm{E}+02$ & $2.18 \mathrm{E}+02$ & $2.77 \mathrm{E}-03$ & \\
\hline Eu-156 & $3.39 \mathrm{E}-11$ & $2.98 \mathrm{E}-11$ & & \\
\hline $\mathrm{Fe}-55$ & $1.83 \mathrm{E}+02$ & $1.61 \mathrm{E}+02$ & $8.99 \mathrm{E}-06$ & \\
\hline $\mathrm{H}-3$ & $4.68 \mathrm{E}+01$ & $4.12 \mathrm{E}+01$ & $2.81 \mathrm{E}+02$ & $1.52 \mathrm{E}+01$ \\
\hline I-129 & $3.25 \mathrm{E}-02$ & $2.86 \mathrm{E}-02$ & $4.16 \mathrm{E}-06$ & \\
\hline I-129_KB & & & & $1.78 \mathrm{E}-02$ \\
\hline $\mathrm{Kr}-85$ & $7.17 \mathrm{E}+02$ & $6.31 \mathrm{E}+02$ & & \\
\hline
\end{tabular}




\begin{tabular}{|c|c|c|c|c|}
\hline \multicolumn{5}{|c|}{ Table A.6. Projected Inventory for Cement-stabilized Encapsulated Waste } \\
\hline $\begin{array}{l}\text { Radio- } \\
\text { Nuclide }\end{array}$ & $\begin{array}{l}\text { Single Used } \\
\text { Equipment } \\
\text { Storage Area }\end{array}$ & $\begin{array}{c}\text { Combined Use } \\
\text { Equipment } \\
\text { Storage Area } \\
\text { with } 55 \% \\
\text { Reduction }^{\mathrm{a}}\end{array}$ & Disposed & $\begin{array}{c}\text { Projected } \\
\mathrm{K} \text { and L Basin } \\
\text { Resins }\end{array}$ \\
\hline & $\begin{array}{c}\text { Actual } \\
\text { Inventory }\end{array}$ & Actual Inventory & Inventory & Inventory \\
\hline & $(\mathrm{Ci})$ & $(\mathrm{Ci})$ & $(\mathrm{Ci})$ & $(\mathrm{Ci})$ \\
\hline La-140 & $3.39 \mathrm{E}-11$ & $2.98 \mathrm{E}-11$ & & \\
\hline $\mathrm{Nb}-95$ & $1.45 \mathrm{E}-09$ & $1.28 \mathrm{E}-09$ & & \\
\hline $\mathrm{Nb}-95 \mathrm{~m}$ & $3.24 \mathrm{E}-04$ & $2.85 \mathrm{E}-04$ & & \\
\hline $\mathrm{Ni}-59$ & $1.28 \mathrm{E}+00$ & $1.13 \mathrm{E}+00$ & $3.20 \mathrm{E}-04$ & \\
\hline Ni-63 & $1.82 \mathrm{E}+02$ & $1.60 \mathrm{E}+02$ & 3.34E-03 & \\
\hline $\mathrm{Np}-237$ & & & 4.99E-04 & $9.68 \mathrm{E}-01$ \\
\hline Pd-107 & $9.63 \mathrm{E}-03$ & $8.47 \mathrm{E}-03$ & $6.76 \mathrm{E}-06$ & \\
\hline $\mathrm{Pa}-233$ & & & $5.42 \mathrm{E}-06$ & \\
\hline $\mathrm{Pa}-234 \mathrm{~m}$ & & & $5.73 \mathrm{E}-04$ & \\
\hline Pm-147 & $2.43 \mathrm{E}+03$ & $2.24 \mathrm{E}+03$ & $5.45 \mathrm{E}-04$ & \\
\hline Pm-148 & $3.39 \mathrm{E}-11$ & $2.98 \mathrm{E}-11$ & & \\
\hline Pm-148m & $1.89 \mathrm{E}-10$ & $1.66 \mathrm{E}-10$ & & \\
\hline Pr-143 & $3.39 \mathrm{E}-11$ & $2.98 \mathrm{E}-11$ & & \\
\hline Pr-144 & $1.14 \mathrm{E}+03$ & $1.00 \mathrm{E}+03$ & $5.58 \mathrm{E}-03$ & \\
\hline Pr-144m & & & $7.73 \mathrm{E}-07$ & \\
\hline $\mathrm{Pu}-238$ & $8.93 \mathrm{E}-01$ & $7.86 \mathrm{E}-01$ & $1.10 \mathrm{E}-01$ & $9.87 \mathrm{E}-01$ \\
\hline $\mathrm{Pu}-239$ & $8.68 \mathrm{E}-02$ & $7.64 \mathrm{E}-02$ & $4.24 \mathrm{E}-02$ & $2.77 \mathrm{E}+00$ \\
\hline $\mathrm{Pu}-240$ & $2.02 \mathrm{E}-02$ & $1.78 \mathrm{E}-02$ & $1.67 \mathrm{E}-02$ & \\
\hline $\mathrm{Pu}-241$ & $8.00 \mathrm{E}-01$ & $7.04 \mathrm{E}-01$ & 1.99E-01 & $6.18 \mathrm{E}+00$ \\
\hline $\mathrm{Pu}-242$ & & & $3.12 \mathrm{E}-05$ & \\
\hline $\mathrm{Rb}-87$ & & & $5.25 \mathrm{E}-12$ & \\
\hline Rh-106 & $1.52 \mathrm{E}+03$ & $1.34 \mathrm{E}+03$ & $1.39 \mathrm{E}-04$ & \\
\hline Ru-103 & $1.26 \mathrm{E}-08$ & $1.11 \mathrm{E}-08$ & & \\
\hline Ru-103m & $1.26 \mathrm{E}-08$ & $1.11 \mathrm{E}-08$ & & \\
\hline Ru-106 & $1.52 \mathrm{E}+03$ & $1.34 \mathrm{E}+03$ & $1.39 \mathrm{E}-04$ & \\
\hline Sb-125 & $2.23 \mathrm{E}+02$ & $1.96 \mathrm{E}+02$ & $1.51 \mathrm{E}-03$ & \\
\hline Sb-126 & & & $1.08 \mathrm{E}-05$ & \\
\hline Sb-126m & & & $1.08 \mathrm{E}-05$ & \\
\hline $\mathrm{Se}-79$ & $3.49 \mathrm{E}-02$ & $3.07 \mathrm{E}-02$ & $3.32 \mathrm{E}-04$ & \\
\hline Sm-151 & $1.05 \mathrm{E}+02$ & $9.24 \mathrm{E}+01$ & $7.59 \mathrm{E}-03$ & \\
\hline Sn-123 & $4.02 \mathrm{E}-04$ & $3.54 \mathrm{E}-04$ & & \\
\hline Sn-126 & $4.78 \mathrm{E}-02$ & $4.21 \mathrm{E}-02$ & $1.55 \mathrm{E}-05$ & \\
\hline Sr-85 & & & $1.28 \mathrm{E}-06$ & \\
\hline Sr-89 & $9.64 \mathrm{E}-07$ & $8.48 \mathrm{E}-07$ & & \\
\hline Sr-90 & $6.03 \mathrm{E}+03$ & $5.31 \mathrm{E}+03$ & $5.26 \mathrm{E}-01$ & $2.27 \mathrm{E}+03$ \\
\hline Tb-160 & $2.71 \mathrm{E}-06$ & $2.39 \mathrm{E}-06$ & & \\
\hline Tc-99 & $1.25 \mathrm{E}+00$ & $1.10 \mathrm{E}+00$ & $2.11 \mathrm{E}-04$ & \\
\hline TC-99_KB & & & & $2.81 \mathrm{E}+00$ \\
\hline Te-125m & $2.19 \mathrm{E}+02$ & $1.93 \mathrm{E}+02$ & & \\
\hline
\end{tabular}




\begin{tabular}{|c|c|c|c|c|}
\hline \multicolumn{5}{|c|}{ Table A.6. Projected Inventory for Cement-stabilized Encapsulated Waste } \\
\hline $\begin{array}{l}\text { Radio- } \\
\text { Nuclide }\end{array}$ & $\begin{array}{l}\text { Single Used } \\
\text { Equipment } \\
\text { Storage Area }\end{array}$ & $\begin{array}{c}\text { Combined Use } \\
\text { Equipment } \\
\text { Storage Area } \\
\text { with } 55 \% \\
\text { Reduction }^{\mathrm{a}}\end{array}$ & Disposed & $\begin{array}{c}\text { Projected } \\
\mathrm{K} \text { and L Basin } \\
\text { Resins }\end{array}$ \\
\hline & $\begin{array}{c}\text { Actual } \\
\text { Inventory }\end{array}$ & Actual Inventory & Inventory & Inventory \\
\hline & $(\mathrm{Ci})$ & $(\mathrm{Ci})$ & $(\mathrm{Ci})$ & $(\mathrm{Ci})$ \\
\hline Te-127 & 3.39E-11 & $2.98 \mathrm{E}-11$ & & \\
\hline Te-127m & $1.28 \mathrm{E}-02$ & $1.13 \mathrm{E}-02$ & & \\
\hline Te-129 & 3.39E-11 & $2.98 \mathrm{E}-11$ & & \\
\hline Te-129m & 3.39E-11 & $2.98 \mathrm{E}-11$ & & \\
\hline Th-231 & & & $6.11 \mathrm{E}-06$ & \\
\hline Th-234 & & & 5.73E-04 & \\
\hline U-232 & $2.65 \mathrm{E}-03$ & $2.33 \mathrm{E}-03$ & $3.27 \mathrm{E}-08$ & \\
\hline U-233 & $9.29 \mathrm{E}-04$ & $8.18 \mathrm{E}-04$ & $5.19 \mathrm{E}-05$ & \\
\hline $\begin{array}{l}\text { U-233 } \\
\text { Depleted }\end{array}$ & & & $7.87 \mathrm{E}-07$ & \\
\hline U-234 & $2.10 \mathrm{E}-01$ & $1.85 \mathrm{E}-01$ & $1.07 \mathrm{E}-03$ & $4.15 \mathrm{E}-01$ \\
\hline U-235 & $1.50 \mathrm{E}-03$ & $1.32 \mathrm{E}-03$ & $2.10 \mathrm{E}-05$ & $8.99 \mathrm{E}-03$ \\
\hline $\begin{array}{l}\text { U-235 } \\
\text { Depleted }\end{array}$ & & & $1.67 \mathrm{E}-06$ & \\
\hline U-236 & $3.89 \mathrm{E}-02$ & $3.42 \mathrm{E}-02$ & 5.33E-05 & \\
\hline U-238 & $1.24 \mathrm{E}-04$ & $1.09 \mathrm{E}-04$ & $6.38 \mathrm{E}-04$ & $3.43 \mathrm{E}-01$ \\
\hline Y-90 & $6.03 \mathrm{E}+03$ & $5.31 \mathrm{E}+03$ & $5.17 \mathrm{E}-01$ & $2.27 \mathrm{E}+03$ \\
\hline Y-91 & $1.98 \mathrm{E}-04$ & $1.74 \mathrm{E}-04$ & & \\
\hline Zr-93 & $1.66 \mathrm{E}-01$ & $1.46 \mathrm{E}-01$ & $1.02 \mathrm{E}-03$ & \\
\hline $\mathrm{Zr}-95$ & $3.24 \mathrm{E}-04$ & $2.85 \mathrm{E}-04$ & & \\
\hline Total & $4.42 \mathrm{E}+04$ & $3.89 \mathrm{E}+04$ & & \\
\hline
\end{tabular}

${ }^{a}$ As per Calculation Number G-CLC-E-00013, Savannah River Site Internal Document. 


\section{Appendix B. PA Replacement Figures}

These tables containing figures replace Figures G-79 through G-90 in the PA. In three cases the fluxes and concentrations are shown as increasing rapidly at 10,000 years. Estimates of the peak concentrations for those three cases based on a comparison with slit trench performance are as follows:

\begin{tabular}{|c|c|c|c|c|c|}
\hline \multirow[b]{2}{*}{ Nuclide } & \multicolumn{3}{|c|}{ Two sets of 5 Slit Trenches } & \multicolumn{2}{|c|}{ Two sets of CIG Trenches } \\
\hline & $\begin{array}{l}\text { Conc. at } \\
10,000 \\
\text { years }(\mathrm{Ci})\end{array}$ & $\begin{array}{l}\text { Maximum } \\
\text { Estimated } \\
\text { Conc. (Ci) }\end{array}$ & $\begin{array}{l}\text { Time of } \\
\text { Maximum } \\
\text { (years) }\end{array}$ & $\begin{array}{l}\text { Conc. at } \\
10,000 \\
\text { years }(\mathrm{Ci})\end{array}$ & $\begin{array}{l}\text { Maximum Estimated } \\
\text { Conc. (Ci) }\end{array}$ \\
\hline $\mathrm{Cm}-246$ & $2.4 \mathrm{E}-8$ & $2.4 \mathrm{E}-8$ & 15,200 & $4.3 \mathrm{E}-18$ & $2.4 \mathrm{E}-8$ \\
\hline $\mathrm{Zr}-93$ & $3.8 \mathrm{E} 1$ & $4.0 \mathrm{E}+1$ & 16,900 & $3.2 \mathrm{E}-3$ & $4.0 \mathrm{E}+1$ \\
\hline Th-232 & $5.1 \mathrm{E}-7$ & $7.5 \mathrm{E}+0$ & 62,200 & $2.6 \mathrm{E}-15$ & $7.5 \mathrm{E}+0$ \\
\hline
\end{tabular}

The post-10,000 year estimates are based on a conservative analytical model. As noted in replacement Table 5.1-7 post-10,000 year estimates will be provided in the next PA revision.

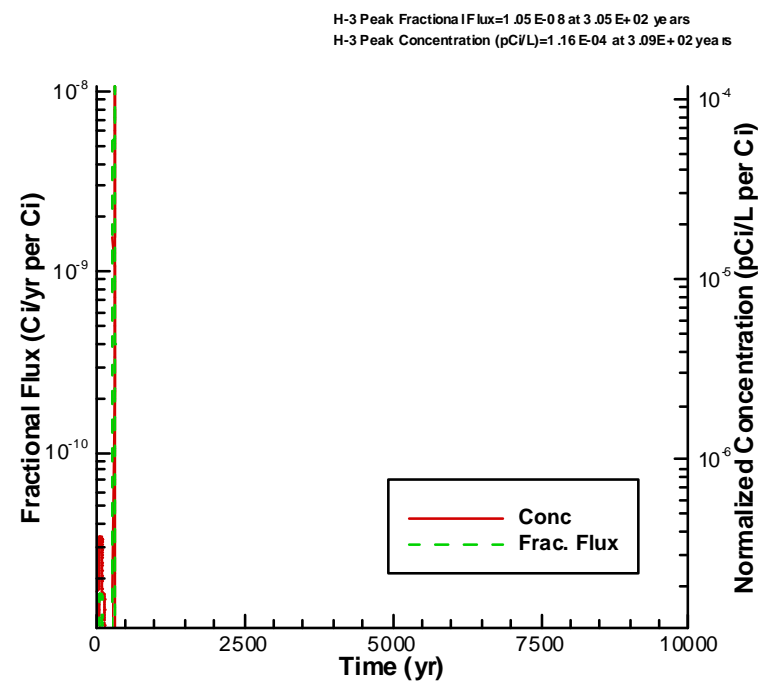

Figure CIG 1. Flux and Concentration for H-3

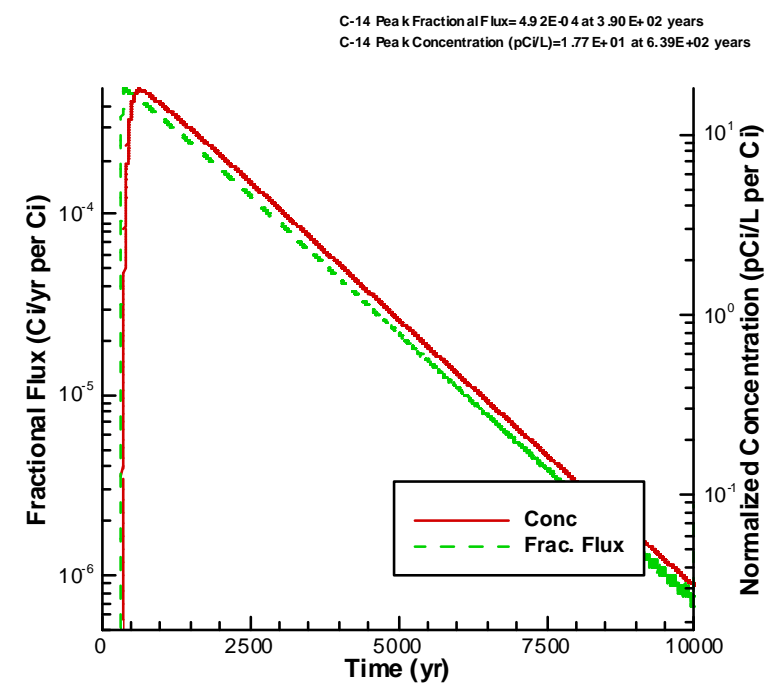

Figure CIG 2. Flux and Concentration for C-14 

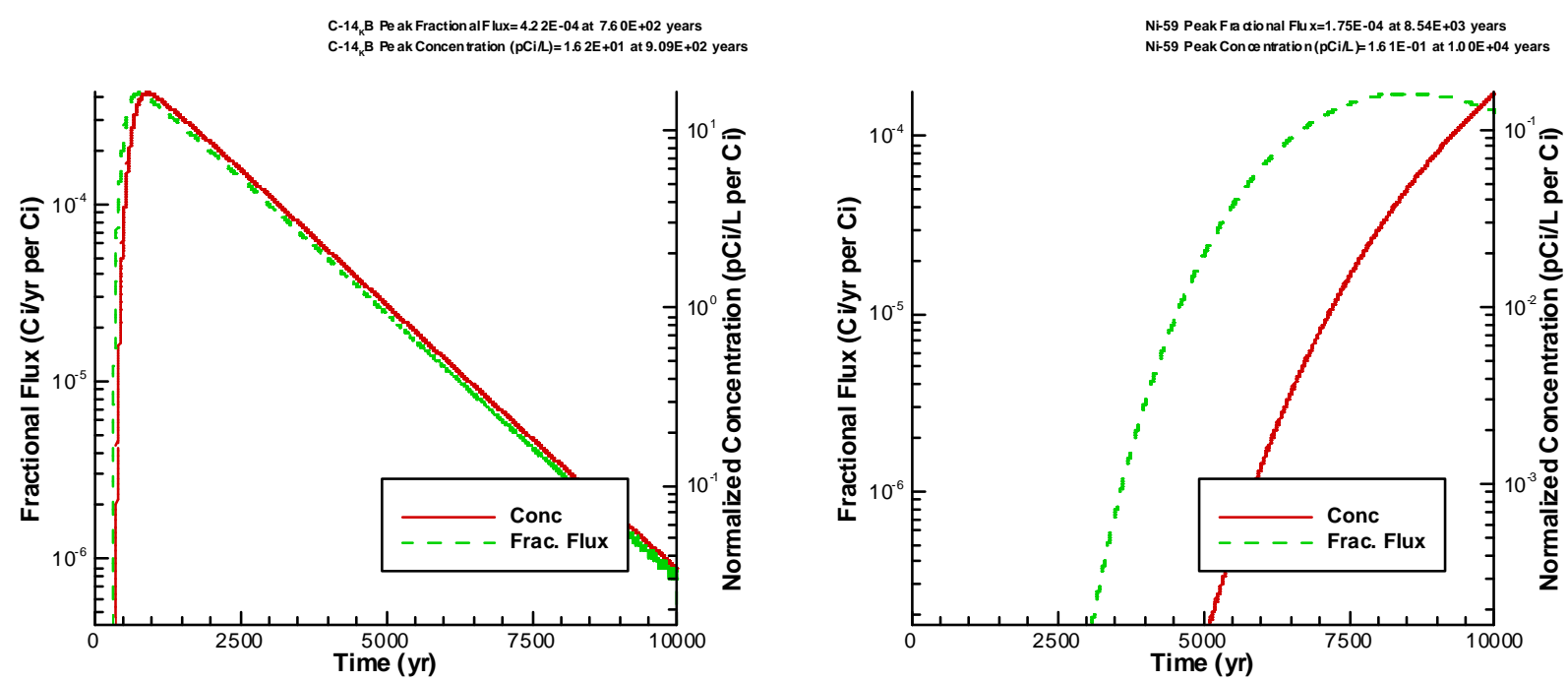

Figure CIG 3. Flux and Concentration for C-14_KB

Figure CIG 4. Flux and Concentration for Ni-59

Se-79 Peak Fractional Flux $=5.49 \mathrm{E}-04$ at $299 \mathrm{E}+03$ yea s

Se-79 Peak Concentration $(\mathrm{pC} / \mathrm{L} L)=3.78 \mathrm{E}+00$ at $7.05 \mathrm{E}+03$ years

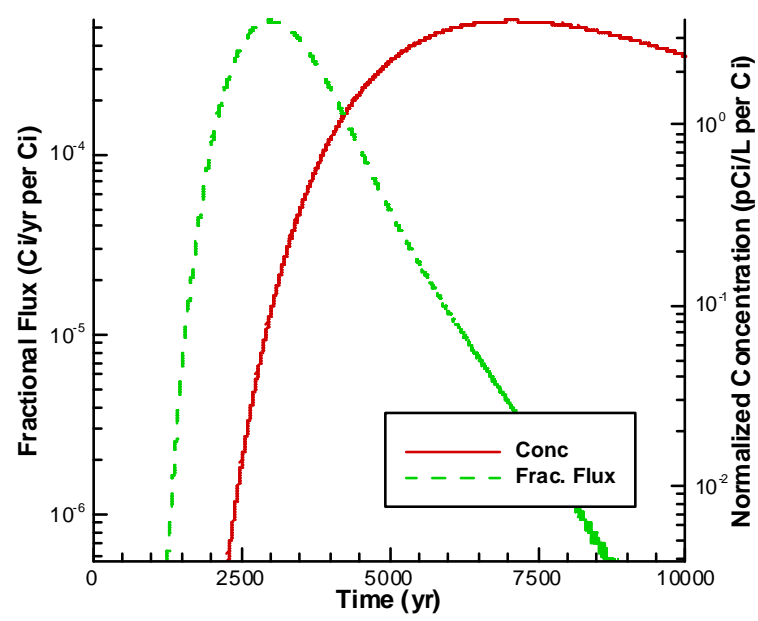

Figure CIG 5. Flux and Concentration for Se-79

SF9 0 Peak F ractional $F u x=1.43 E-07$ at $4.38 E+02$ years SF9 0 Peak $C$ oncentration $(\mathrm{pC} / \mathrm{L})=2.54 \mathrm{E}-05$ at $5.34 \mathrm{E}+02$ years

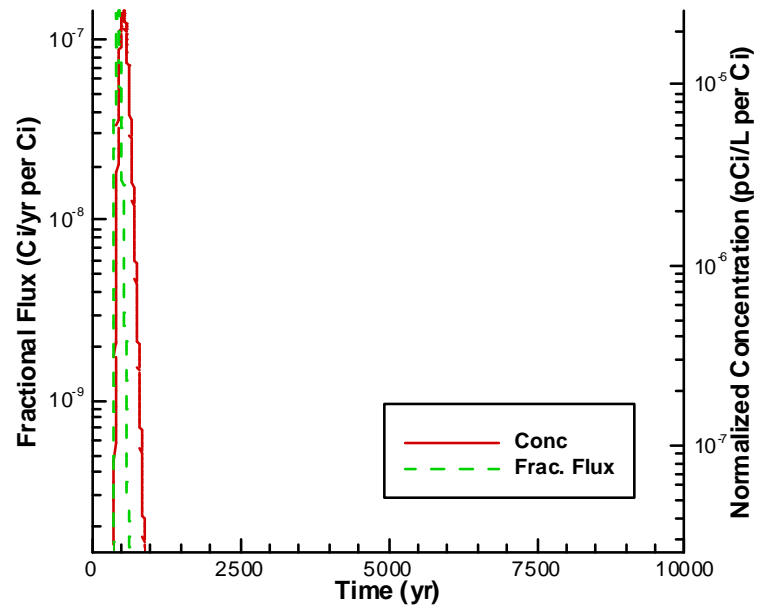

Figure CIG 7. Flux and Concentration for Sr-90

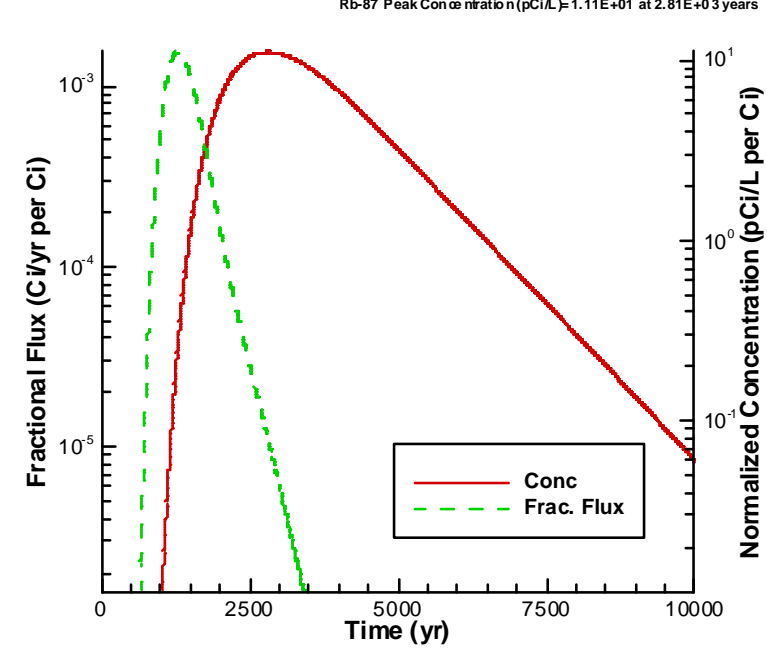

Figure CIG 6. Flux and Concentration for Rb-87

TC-99 Peak Fractional Flu $x=1.32 \mathrm{E}-01$ at $3.12 \mathrm{E}+02$ years
Tc-99 Peak Con $œ$ ntration $(\mathrm{pCC} / \mathrm{L})=1.20 \mathrm{E}+03$ at $3.26 \mathrm{E}+02$ yea

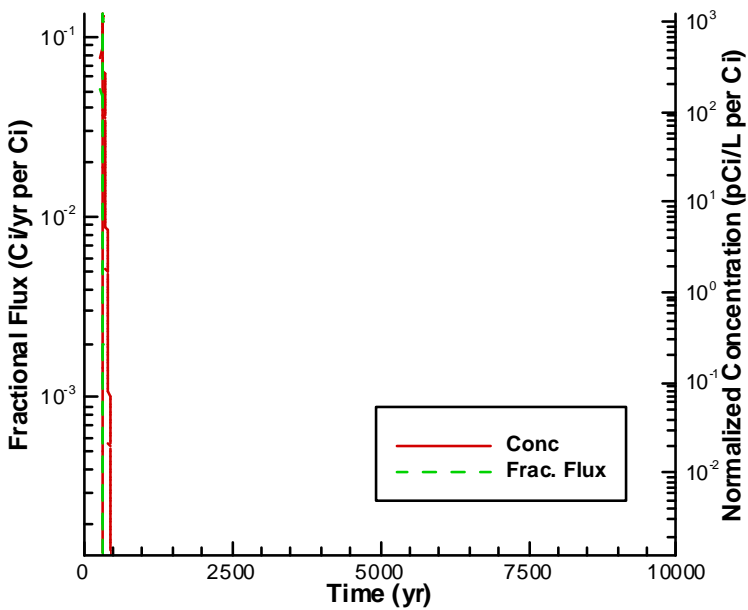

Figure CIG 8. Flux and Concentration for Tc-99 


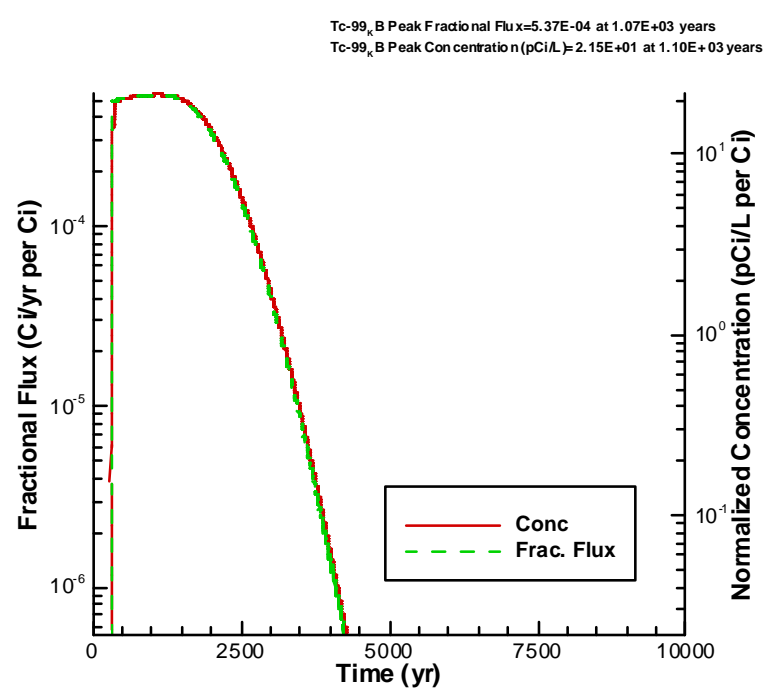

Figure CIG 9. Flux and Concentration for Tc-99_KB Sn-126 Peak Fractional Flu $x=5.65 \mathrm{E}-04$ at $282 \mathrm{E}+03$ years Sn-126 Peak Concen ratio $n(P C i L)=4.43 E+00$ at $6.44 E+03$ years

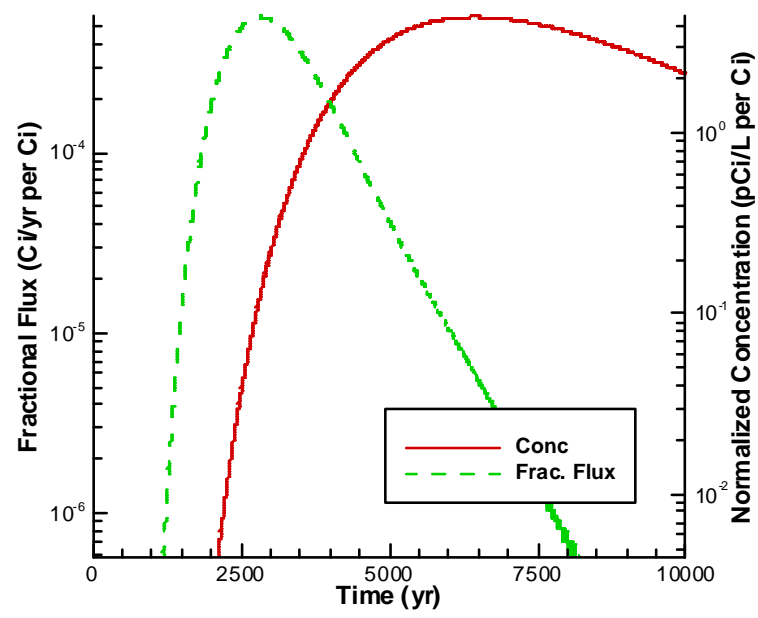

Figure CIG 11. Flux and Concentration for Sn-126 $1-129_{\mathrm{A}}$ Pea $\mathrm{k}$ Fraction al F lux $=1.36 \mathrm{E}-03$ at $6.17 \mathrm{E}+02$ years $1-129_{A}$ Pea k Concentration $(p C i L)=5.45 \mathrm{E}+01$ at $6.62 \mathrm{E}+02$ years

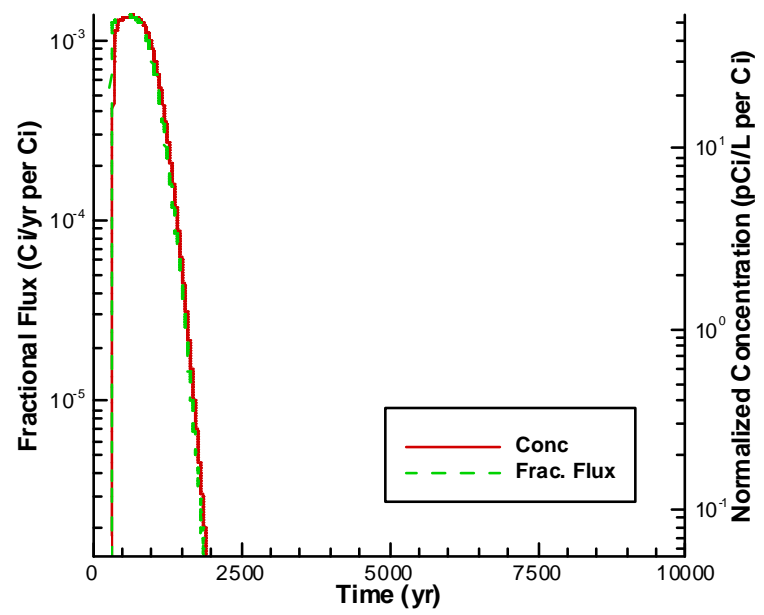

Pd-107 Peak Fractional Flu $x=1.53 \mathrm{E}-03$ at $1.32 \mathrm{E}+03$ years
Pd-107 Peak Concen tration $(\mathrm{pCC} / \mathrm{L})=1.1 \mathrm{E}+01$ at $2.83 \mathrm{E}+03$ year

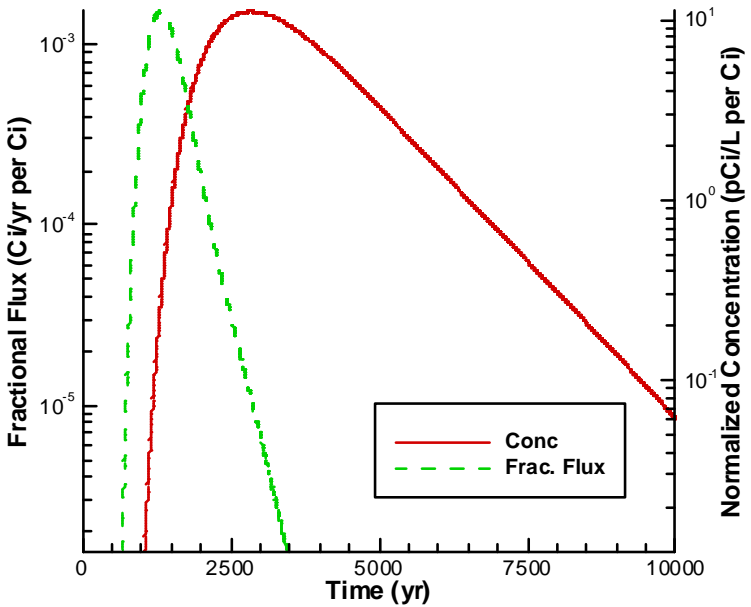

Figure CIG 10. Flux and Concentration for Pd-107

1129 Peak Fractional F lux $=9.53 \mathrm{E}-02$ at $3.17 \mathrm{E}+02$ years

1.129 Peak Concentration $(\mathrm{pC} / \mathrm{L} / \mathrm{L})=8.15 \mathrm{E}+02$ at $3.37 \mathrm{E}+02$ years

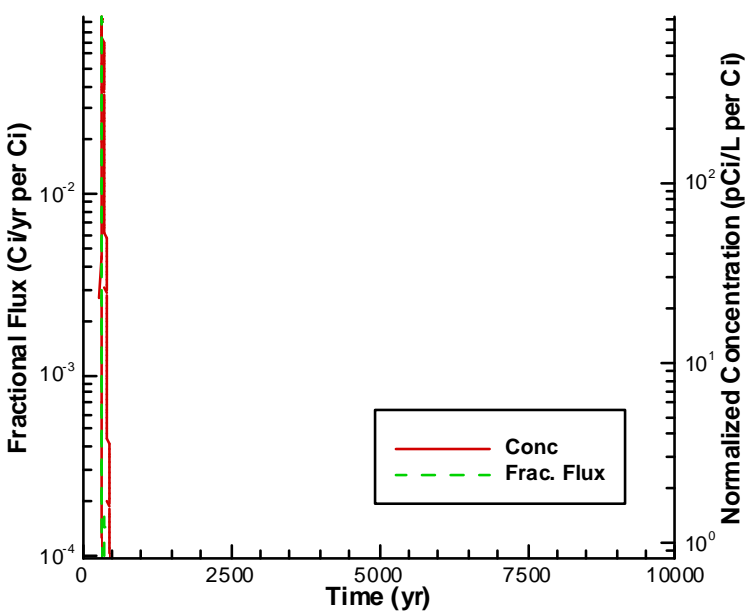

Figure CIG 12. Flux and Concentration for I-129

$1-129_{\mathrm{B}}$ Peak Fractio $+129_{\mathrm{B}}$ Pea $\mathrm{k}$ Concentration $(\mathrm{pC} / \mathrm{L} L)=1.98 \mathrm{E}+01$ at $1.19 \mathrm{E}+03$ years

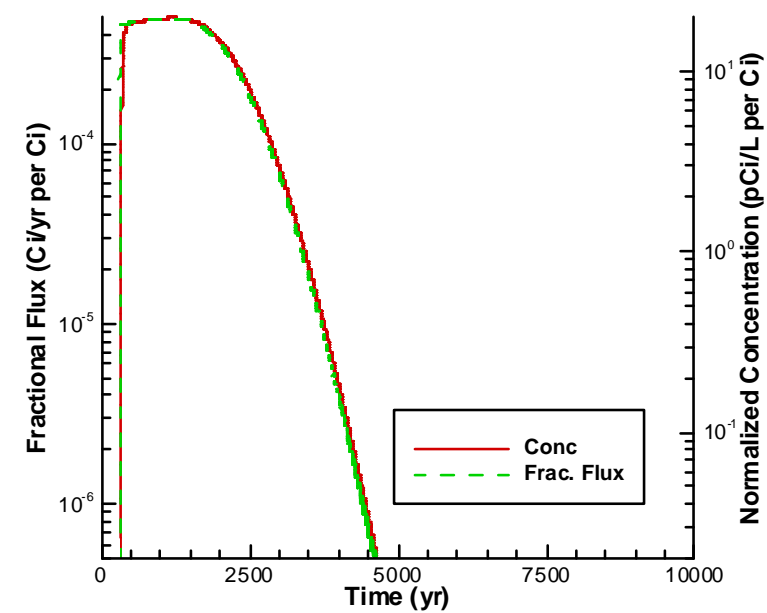


Figure CIG 13. Flux and Concentration for I-129_A

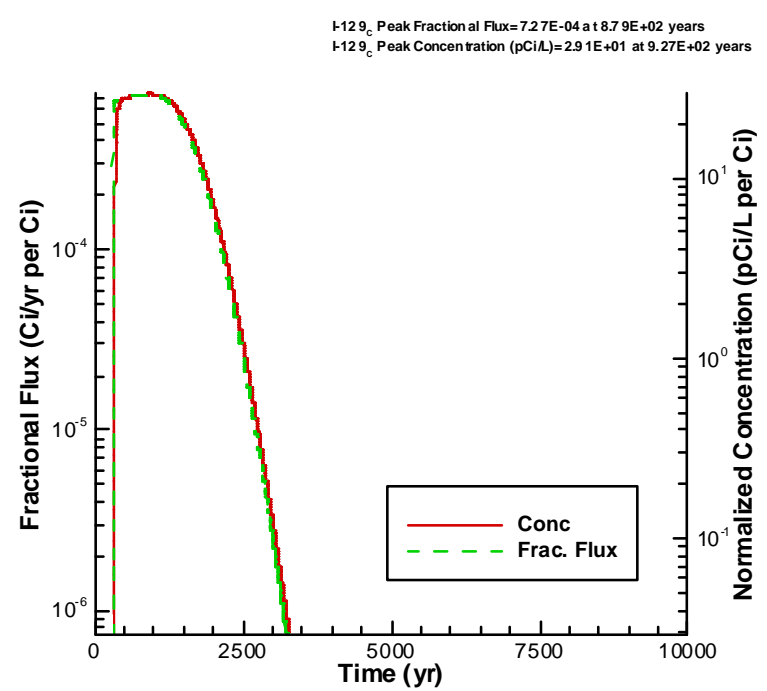

Figure CIG 15. Flux and Concentration for I-129_C

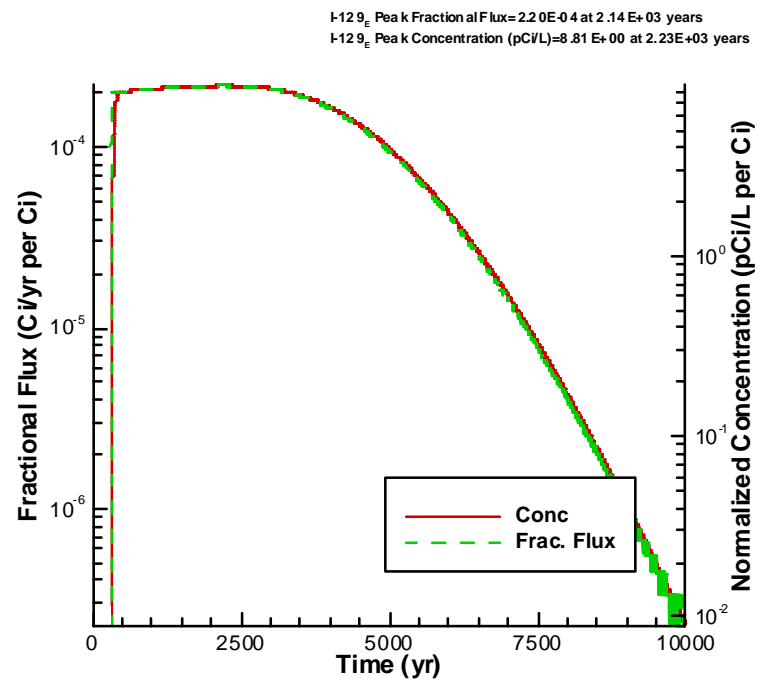

Figure CIG 17. Flux and Concentration for I-129_E
Figure CIG 14. Flux and Concentration for I-129_B

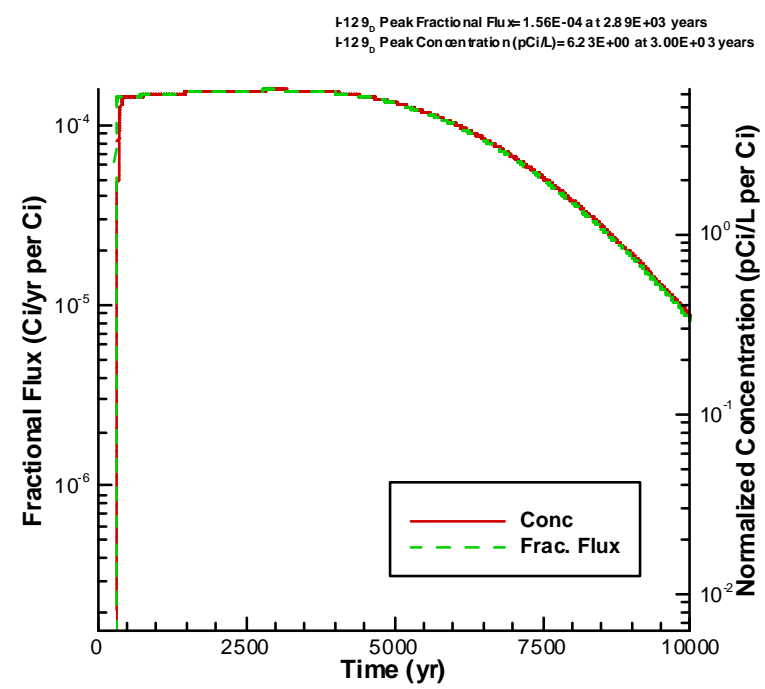

Figure CIG 16. Flux and Concentration for I-129_D

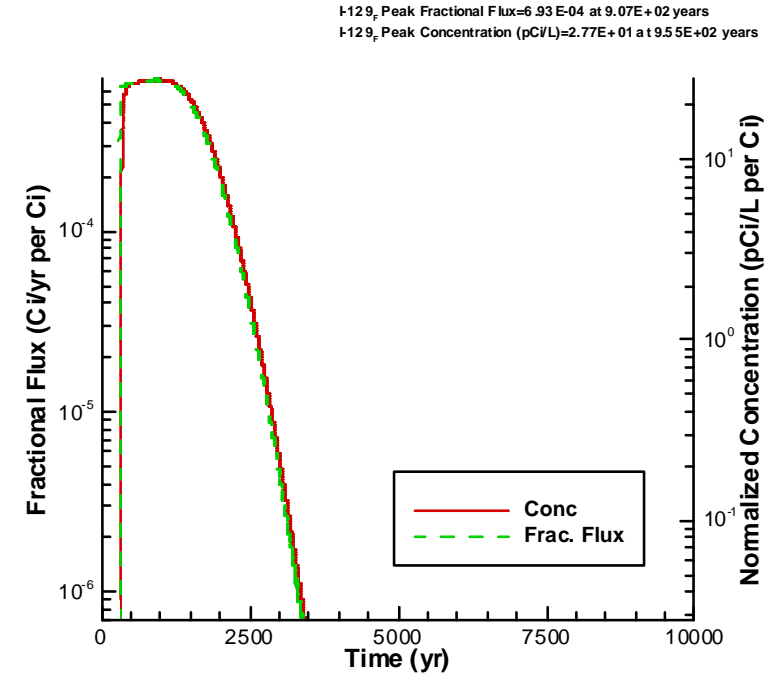

Figure CIG 18. Flux and Concentration for I-129_F 
$1-129_{\mathrm{G}}$ Peak Fradio nal Flu $\mathrm{x}=7.94 \mathrm{E}-02$ at $3.20 \mathrm{E}+02$ yea $1-129_{\mathrm{G}}$ Peak Con centration $(\mathrm{PCi} / \mathrm{L})=8.01 \mathrm{E}+02$ at $3.40 \mathrm{E}+02$ years

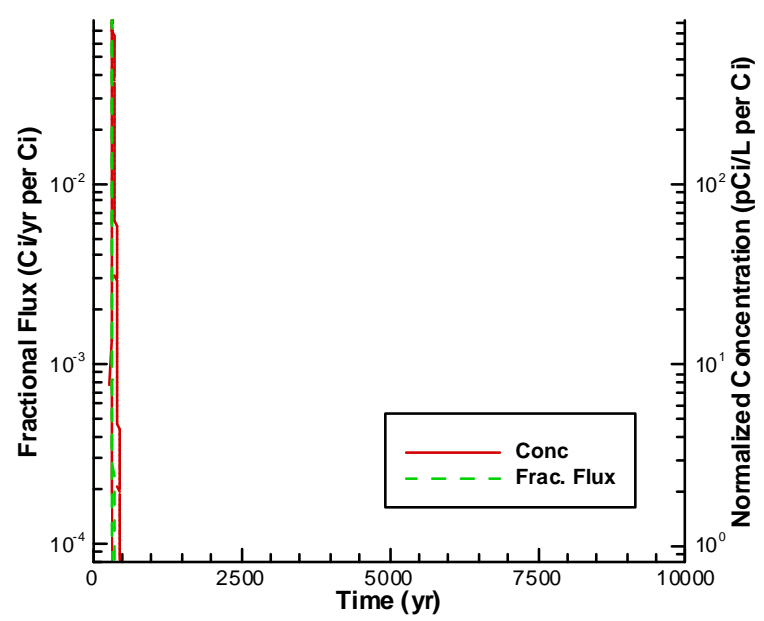

Figure CIG 19. Flux and Concentration for I-129_G

$1-129$, Peak F ractional Flux $=1.41 \mathrm{E}-04$ at $3.15 \mathrm{E}+03$ years 1-129, Peak $C$ oncentration $(\mathrm{pC} / \mathrm{L} L)=5.63 \mathrm{E}+00$ at $328 \mathrm{E}+03$ years

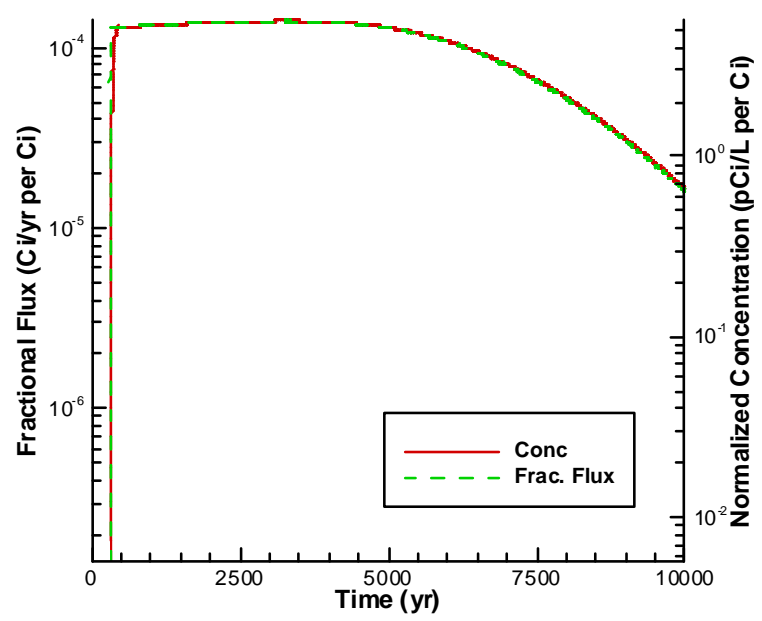

Figure CIG 21. Flux and Concentration for I-129_I

$129_{\mathrm{K}} \mathrm{B}$ Peak Fractional Flux $=1.18 \mathrm{E}-04$ at $3.80 \mathrm{E}+03$ yea is $-129_{k} B$ Peak Concentration $(p C / L)=4.71 E+00$ at $3.85 E+03$ years

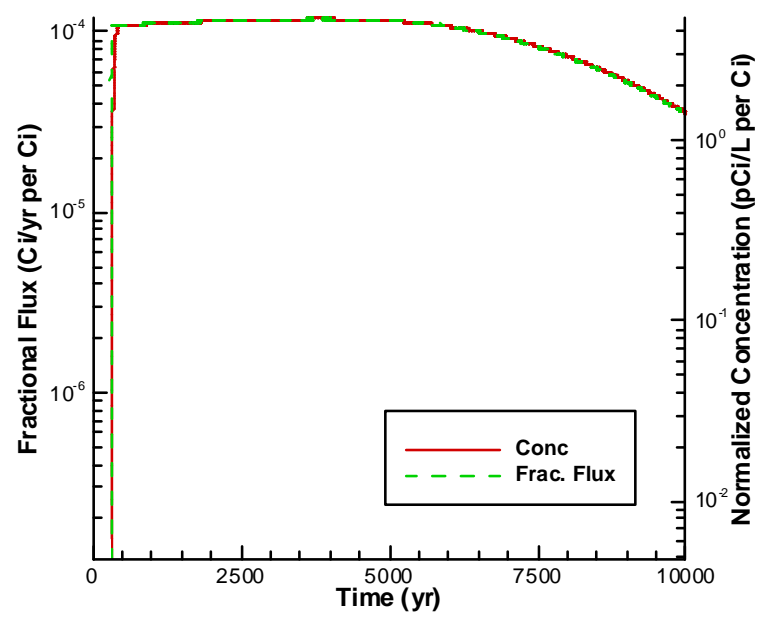

Figure CIG 23. Flux and Concentration for I-129_KB

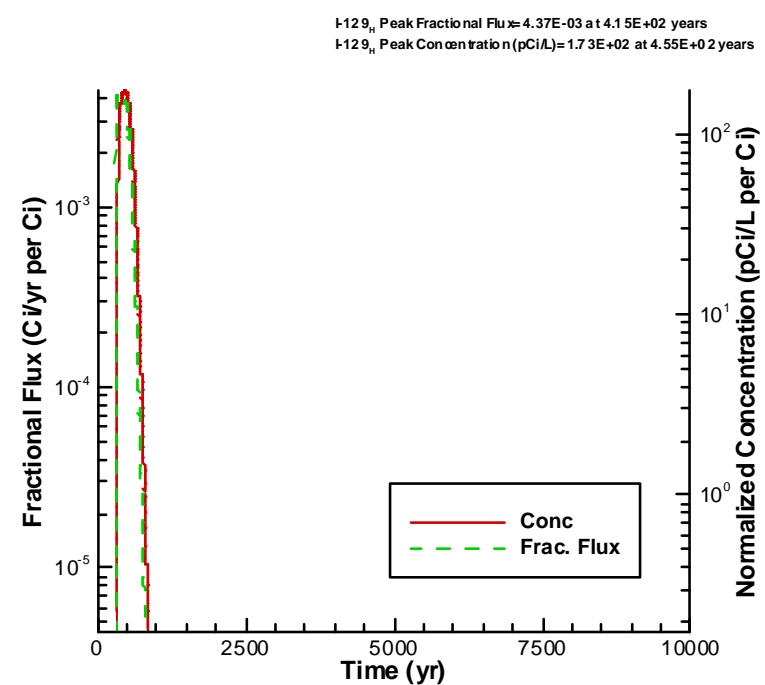

Figure CIG 20. Flux and Concentration for I-129_H

129 PeakFraciona 7 ux $=3.31 \mathrm{E}-02$ at $3.31 \mathrm{E}+02$ years

$1-129$ Peak Concenta $\mathrm{f}$ on $(\mathrm{pC} \mathrm{C})=6.75 \mathrm{E}+02$ at $3.66 \mathrm{E}+02$ years

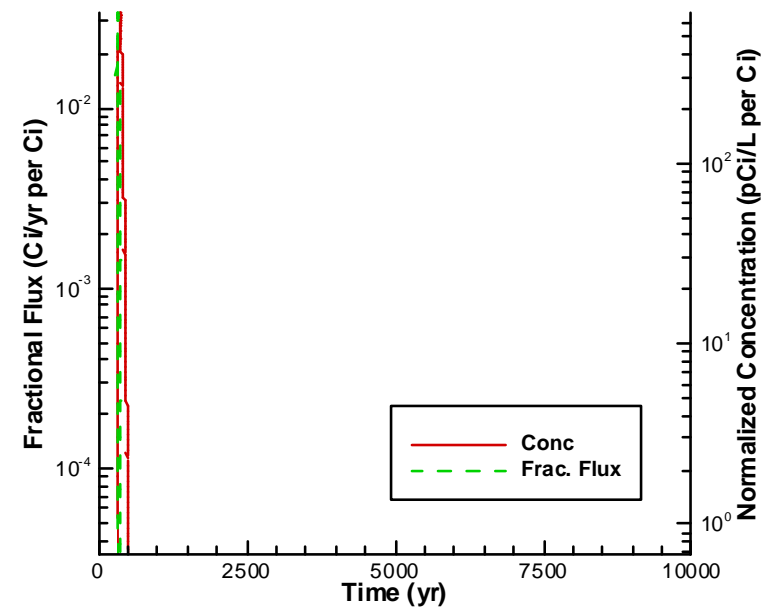

Figure CIG 22. Flux and Concentration for I-129_J $129_{\mathrm{s}}$ Peak Concentration $(\mathrm{pC} / \mathrm{L} \mathrm{L})=3.03 \mathrm{E}+00$ at $5.80 \mathrm{E}+03$ years

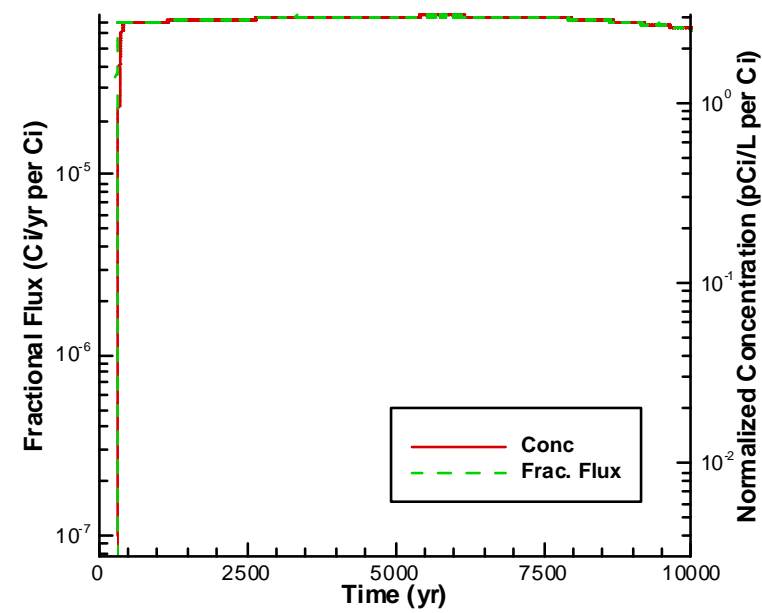

Figure CIG 24. Flux and Concentration for I-129_S 


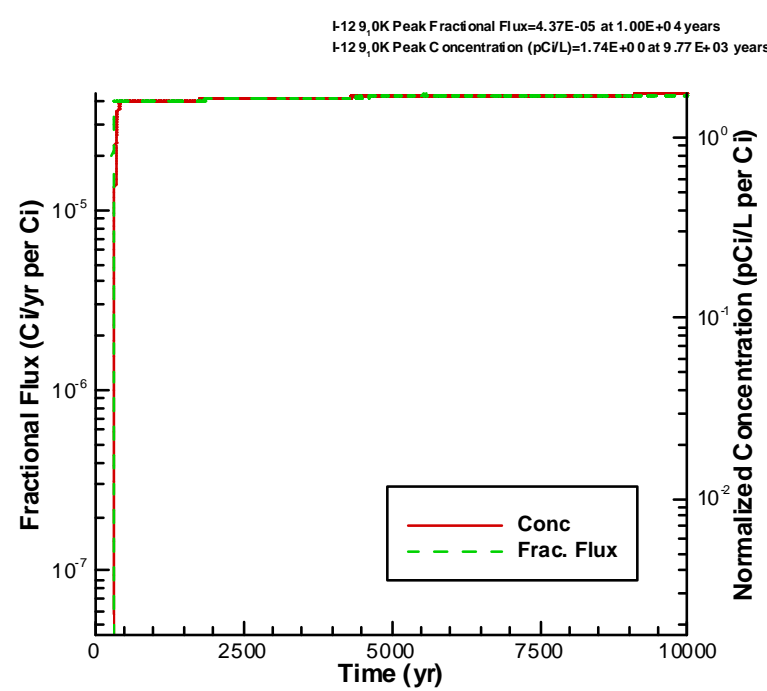

Figure CIG 25. Flux and Concentration for I-129_10K

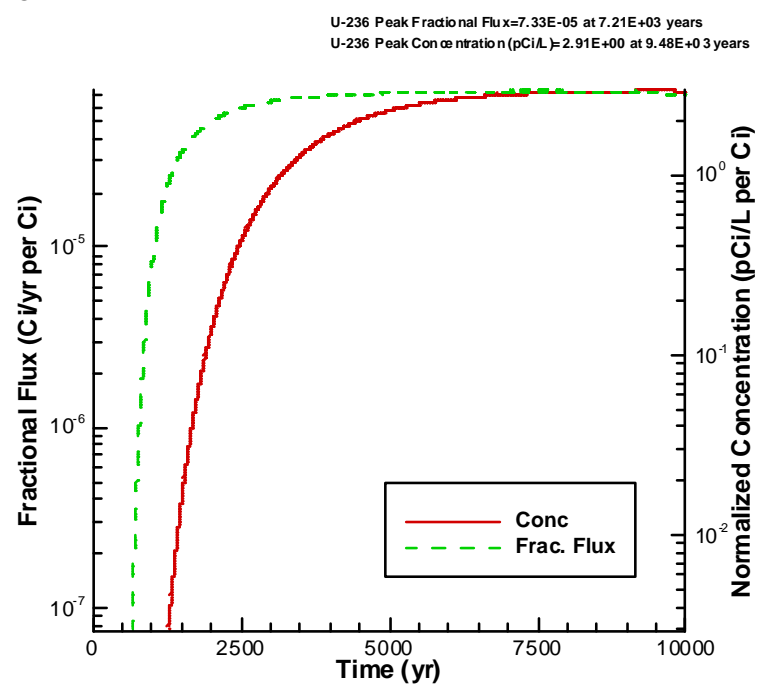

Figure CIG 27. Flux and Concentration for U-236

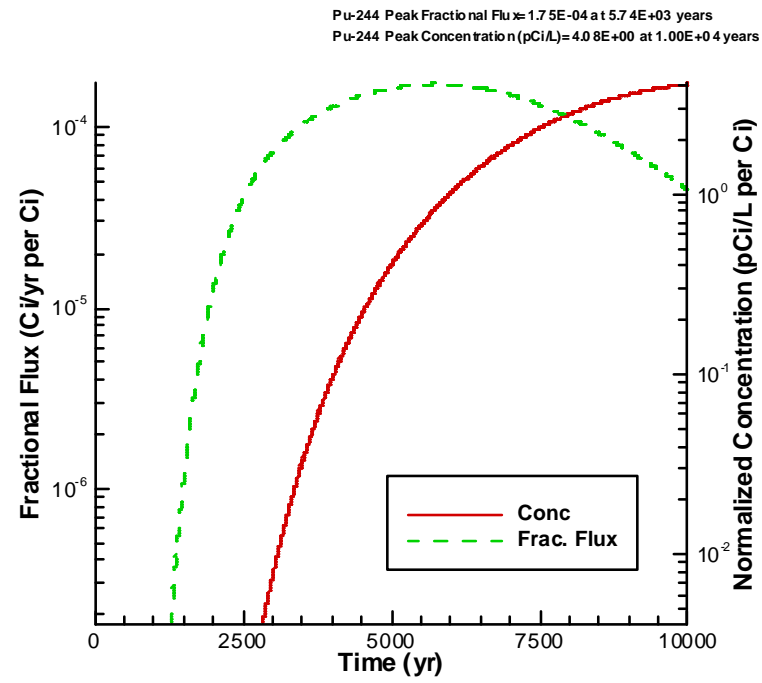

Figure CIG 29. Flux and Concentration for $\mathrm{Pu}-244$

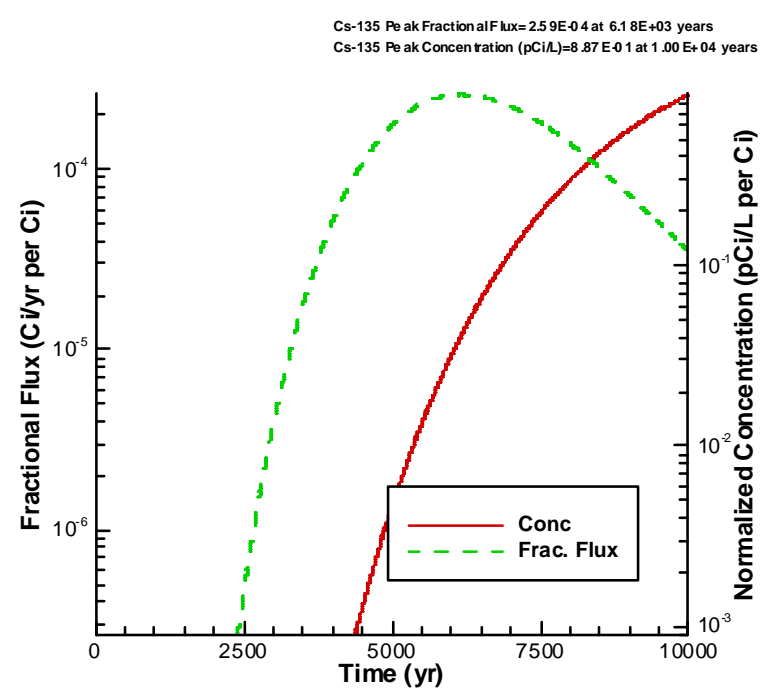

Figure CIG 26. Flux and Concentration for Cs-135

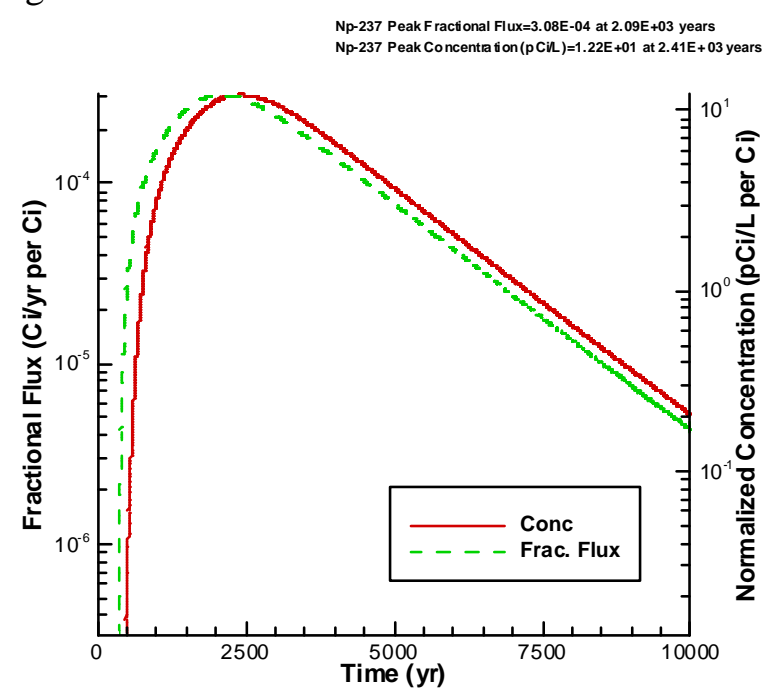

Figure CIG 28. Flux and Concentration for Np-237 Cm-2 46 Pea Fraction alF $F(u x=2.41 \mathrm{E}-15$ at $1.00 \mathrm{E}+04$ years
$\mathrm{Cm} 246$ Peak Concentration $(\mathrm{pCC} / \mathrm{L})=4.30 \mathrm{E}-18$ at $1.00 \mathrm{E}+04$ ye ars

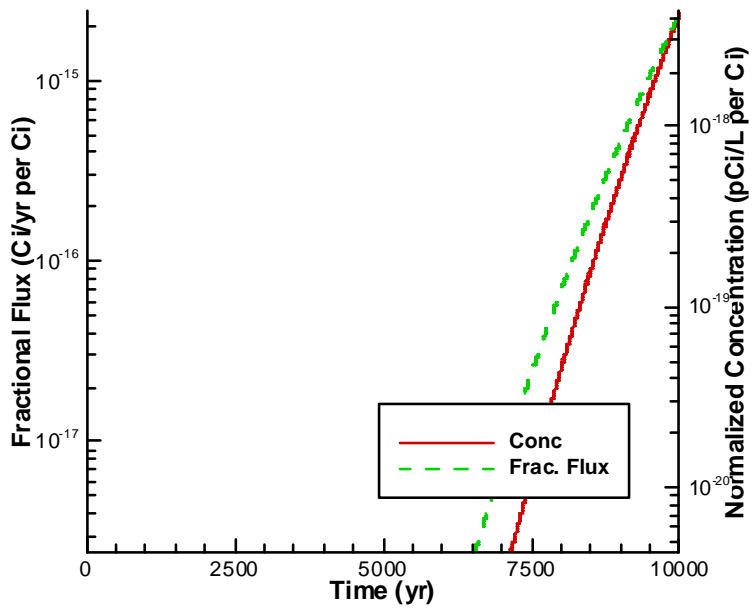

Figure CIG 30. Flux and Concentration for Cm-246 
Zr-93 Peak Conc of $3.23 \mathrm{E}-03$ at $1.00 \mathrm{E}+04$ years
$\mathrm{Nb}-93 \mathrm{~m}$ Peak Conc of $1.26 \mathrm{E}-02$ at $1.00 \mathrm{E}+04$ years

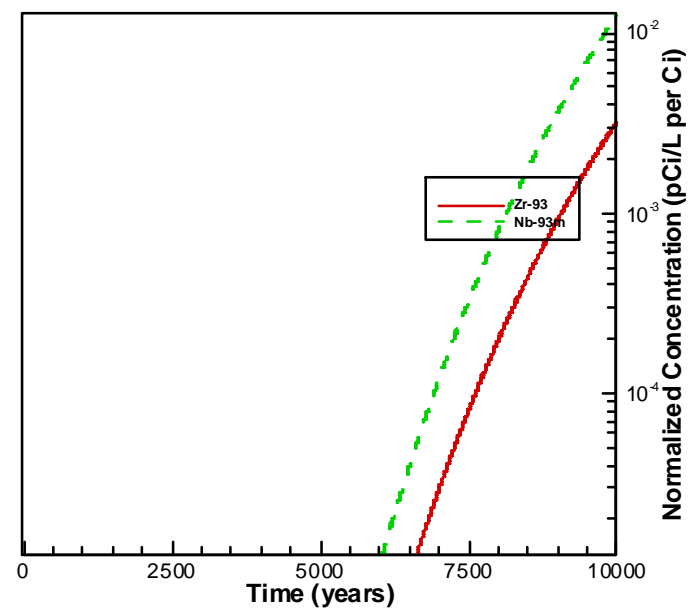

Figure CIG 31. Concentrations for Zr-93

Zr-93 Peak Flux of $8.27 E-05$
Nb-93m PeakFlux of $3.09 E-04$ at $1.00 E+04$ ye ars

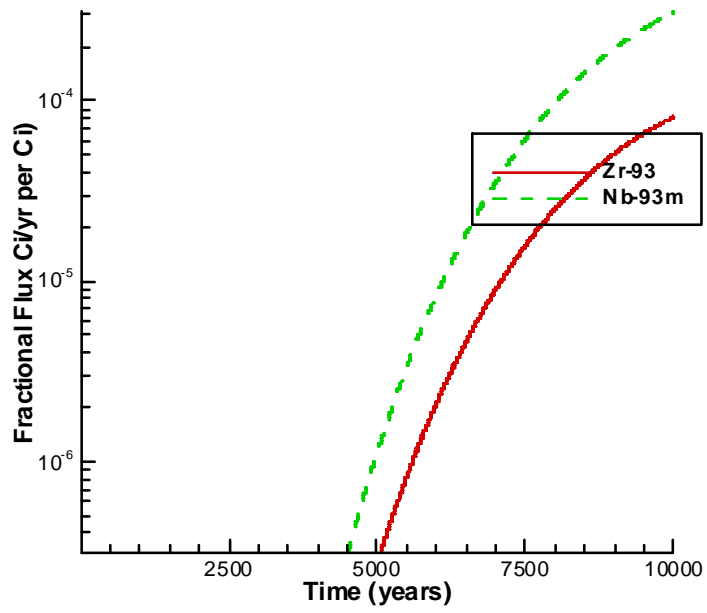

Figure CIG 33. Fluxes for Zr-93
Th-232 Peak Conc of $2.59 \mathrm{E}-15$ at $1.00 \mathrm{E}+04$ years \begin{tabular}{l}
$\mathrm{T} T-228$ \\
$\mathrm{R}$ a-224 Peak Conc of $2.84 \mathrm{E}-15$ at \\
\hline
\end{tabular}

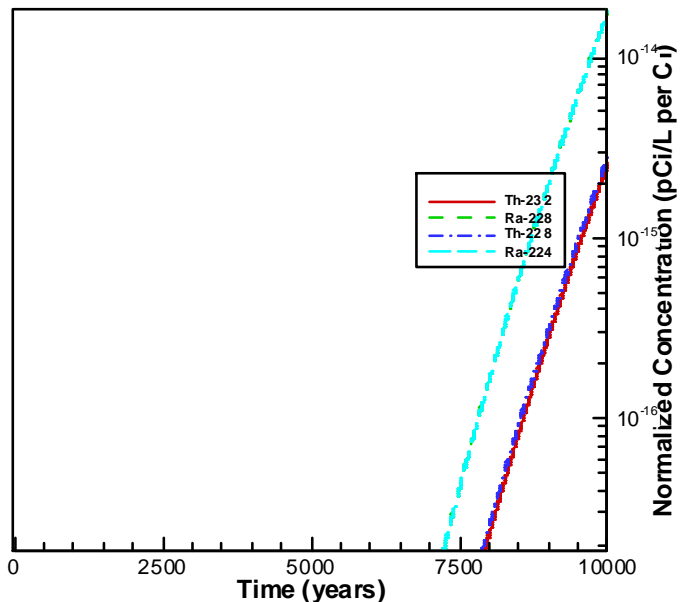

Figure CIG 32. Concentrations for Th-232

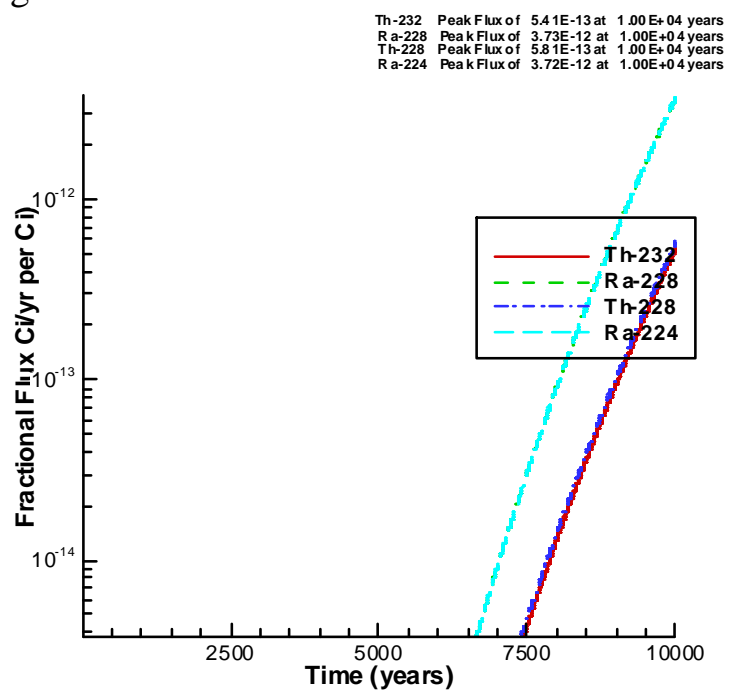

Figure CIG 34. Fluxes for Th-232 
U-232 Peak Conc of $6.62 \mathrm{E}-08$ at $1.18 \mathrm{E}+03$ years

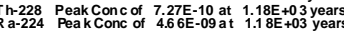

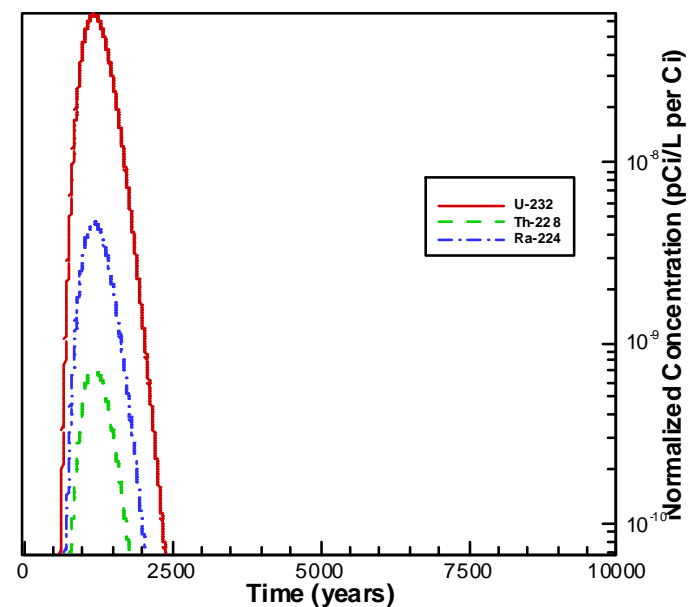

Figure CIG 35. Concentrations for U-232 $\begin{array}{ll}\text { U-232 } & \text { Peak Flux of } 6.64 \mathrm{E}-10 \text { at } \\ \text { Th-22.88 } & \text { Peak }+02 \text { years } \\ \text { Thelux of } 7.32 \mathrm{E}-12 \text { at } & 8.88 \mathrm{E}+02 \text { years }\end{array}$

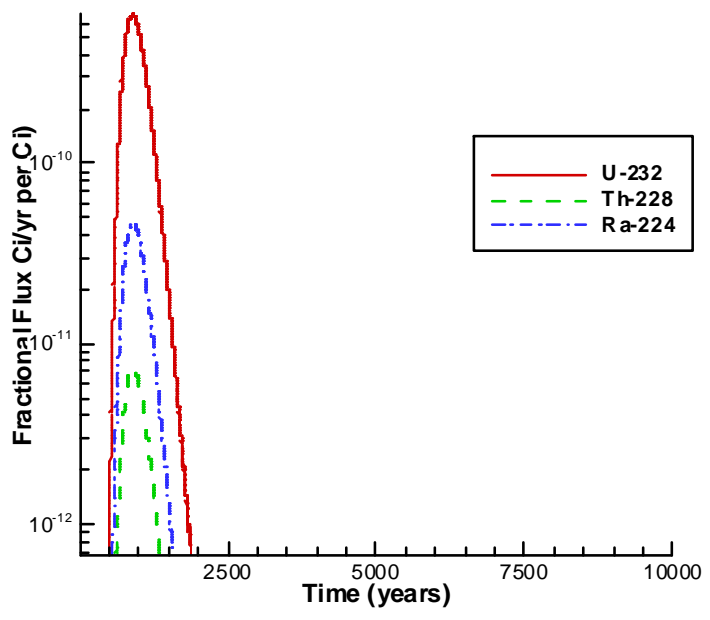

Figure CIG 37. Fluxes for U-232
U-233 Peak Conc of $2.79 E+00$ at $9.14 E+03$ yea is Ra-225 PeakConc of $8.40 \mathrm{E}-02$ at $1.00 \mathrm{E}+04$ years

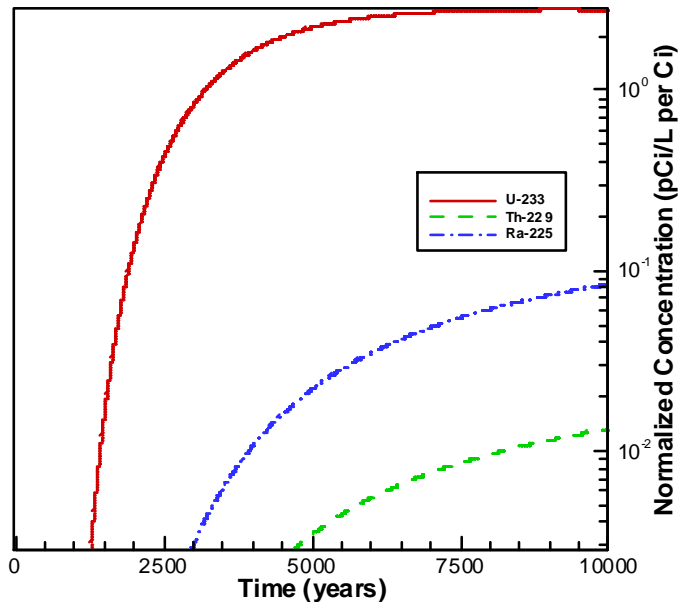

Figure CIG 36. Concentrations for U-233 U-233 Peak Flux of $7.11 \mathrm{E}-05$ at $6.91 \mathrm{E}+03$ years

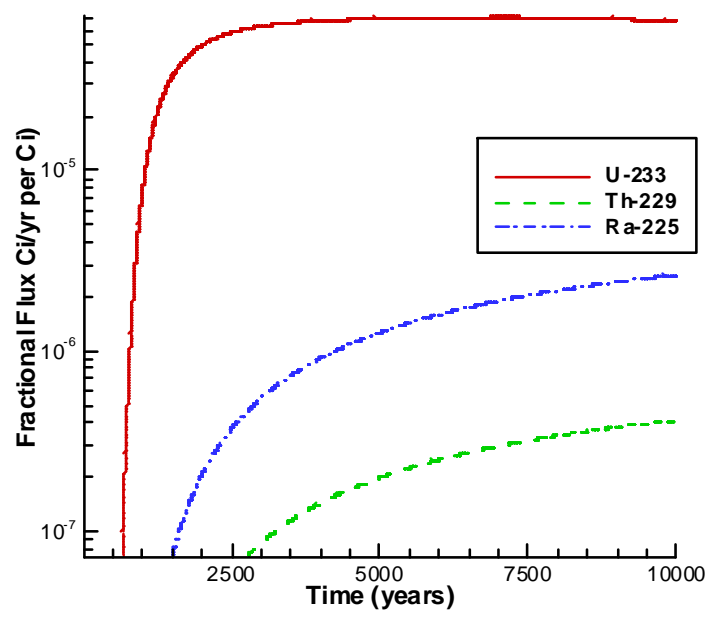

Figure CIG 38. Fluxes for U-233 


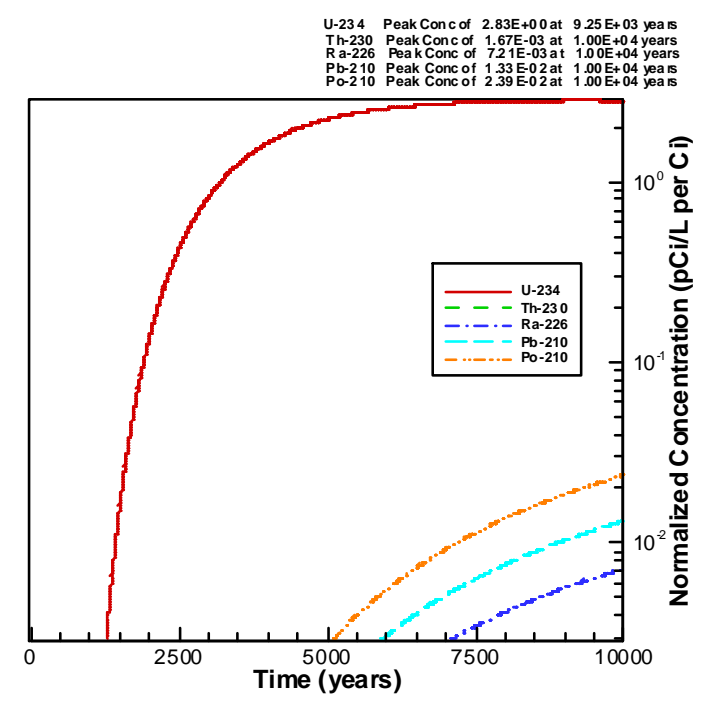

Figure CIG 39. Concentrations for U-234

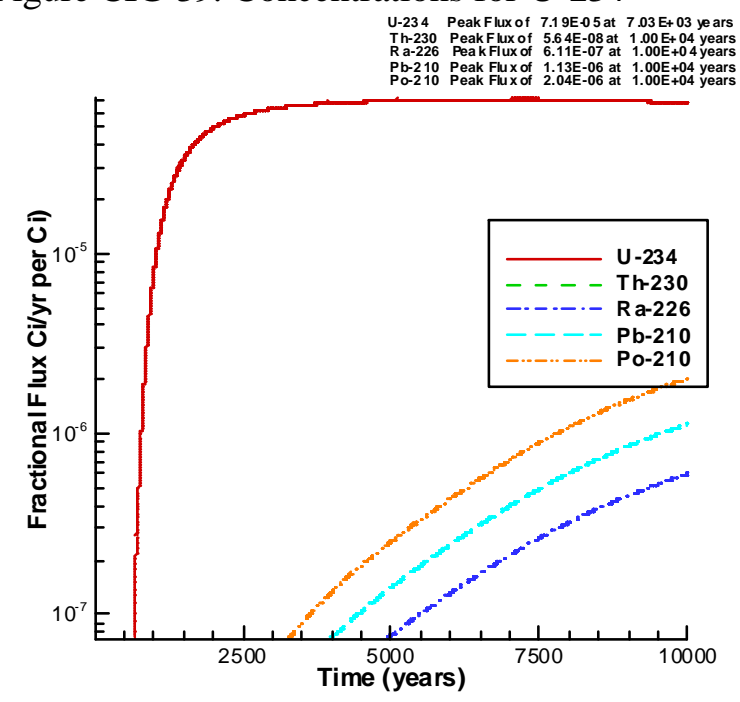

Figure CIG 41. Fluxes for U-234

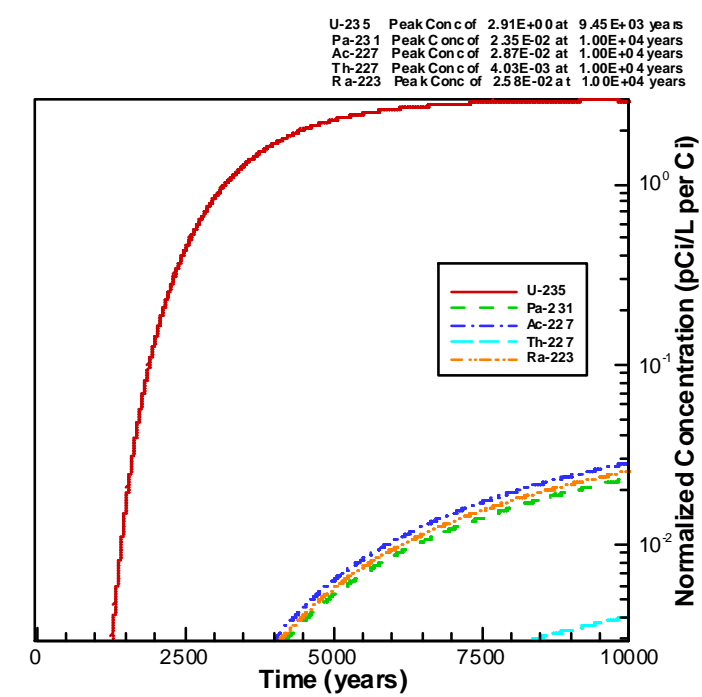

Figure CIG 40. Concentrations for U-235

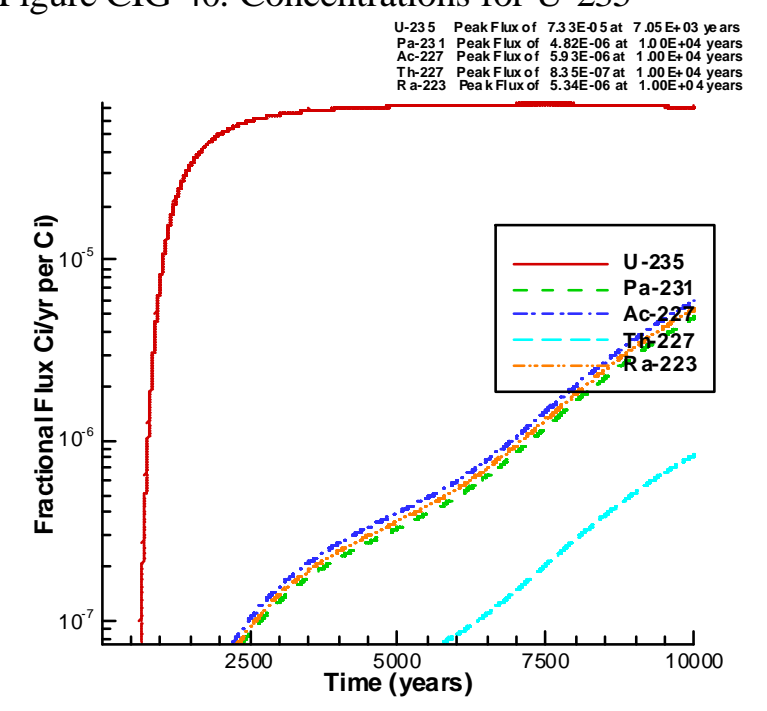

Figure CIG 42. Fluxes for U-235 
WSRC-TR-2003-00318

Rev. 0

U-238 Peak Conc of $4.22 \mathrm{E}-08$ at $9.95 \mathrm{E}+03$ years U-234 Peakconc of $7.64 \mathrm{E}-04$ at $1.00 \mathrm{E}+04$ years

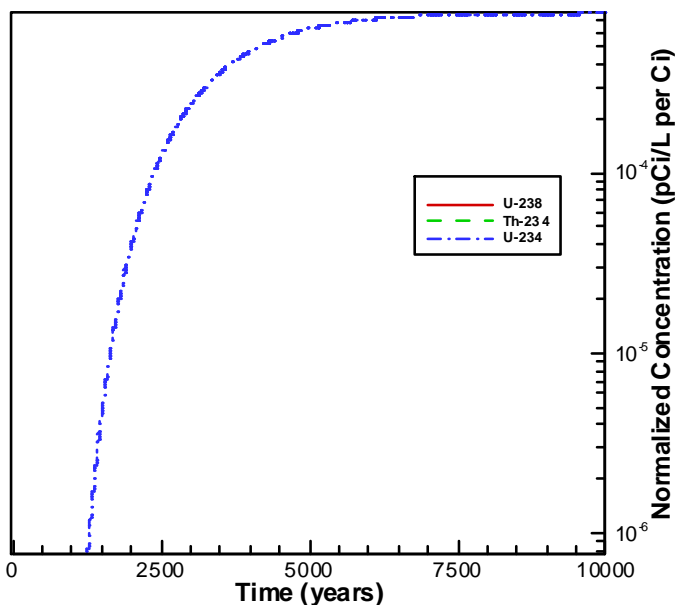

Figure CIG 43. Concentrations for U-238

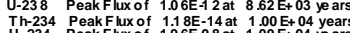

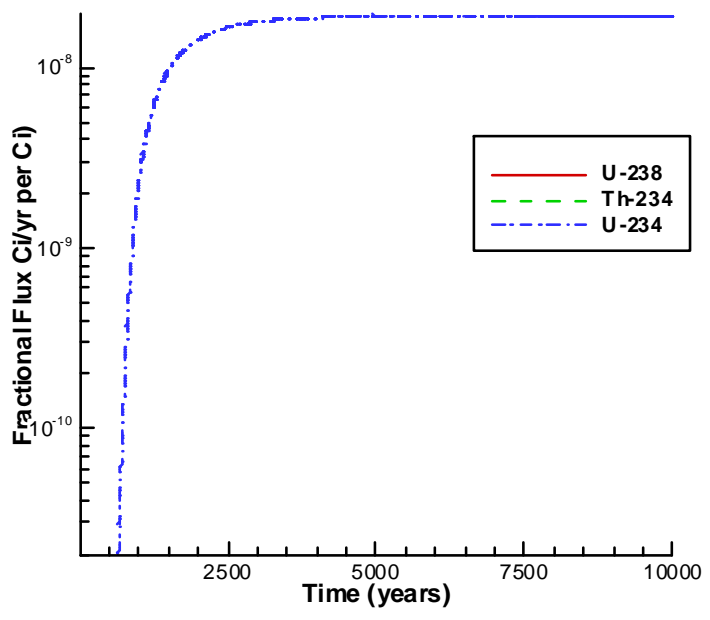

Figure CIG 45. Fluxes for U-238
Pu-238 Peak Conc of $6.29 \mathrm{E}-11$ at $1.85 \mathrm{E}+03$ yea is
U-234 PeakConc of $1.02 \mathrm{E}-03$ at $9.23 \mathrm{E}+03$ years

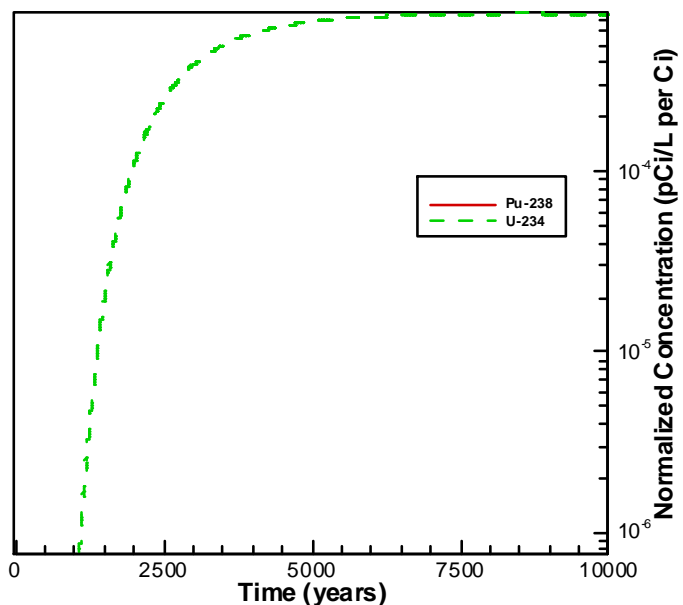

Figure CIG 44. Concentrations for Pu-238 $\begin{array}{ll}\text { Pu-238 } & \text { Peak Flux of } 8.38 \mathrm{E}-12 \text { at } 1.44 \mathrm{E}+03 \text { years } \\ \mathrm{U}-234 & \text { PeakFlux of } 2.58 \mathrm{E}-08 \text { at } \\ 6.67 \mathrm{E}+03 \text { years }\end{array}$

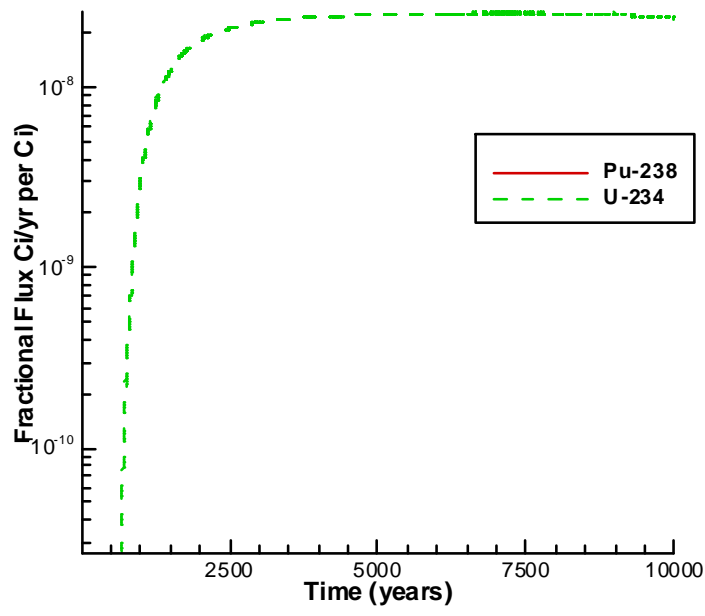

Figure CIG 46. Fluxes for Pu-238 
WSRC-TR-2003-00318

Rev. 0

Pu-239 Peak Conc of $5.47 \mathrm{E}-06$ at $1.00 \mathrm{E}+04$ yea s
$\mathrm{U}-235$ Peak Conc of $2.67 \mathrm{E}-07$ at $9.99 \mathrm{E}+03$ years

Pu-240 Peak Conc of $1.49 \mathrm{E}+00$ at $8.33 \mathrm{E}+03$ years
$\mathrm{U}-236$ Peak Conc of $9.45 \mathrm{E}-04$ at $7.94 \mathrm{E}+03$ years

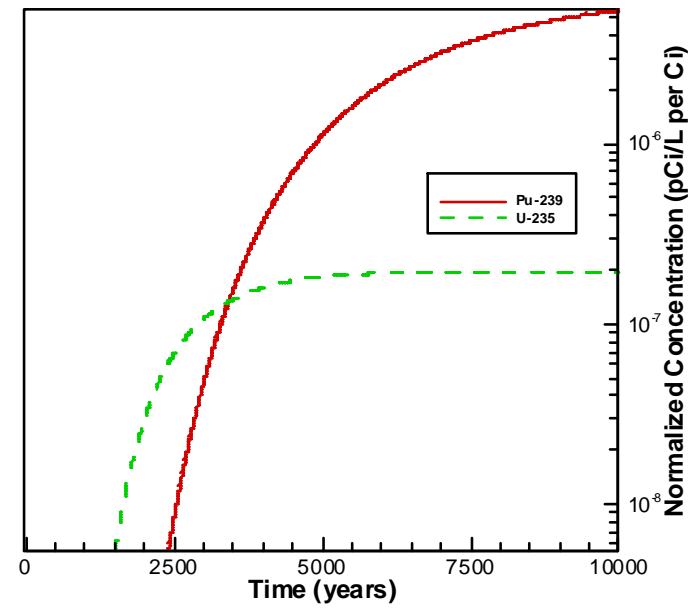

Figure CIG 47. Concentrations for Pu-239

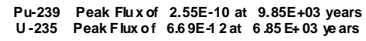

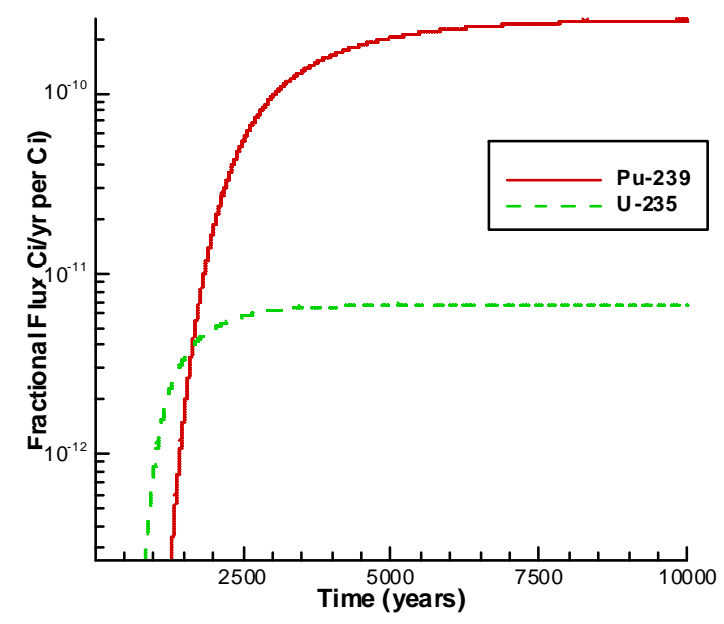

Figure CIG 49. Fluxes for Pu-239

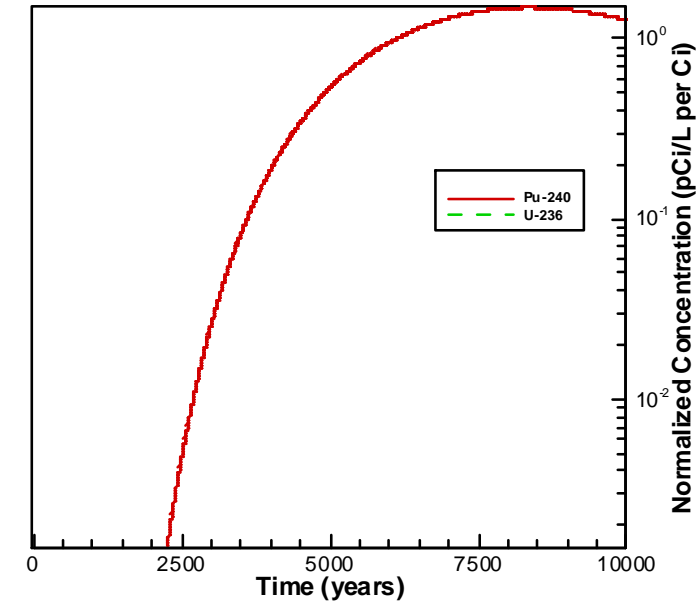

Figure CIG 48. Concentrations for Pu-240 Pu-240 Peak Fux of $9.80 E-05$ at $5.21 E+03$ years
$U-236$
PeakF Fux of $1.82 \mathrm{E}-08$ at $5.12 \mathrm{E}+03$ years

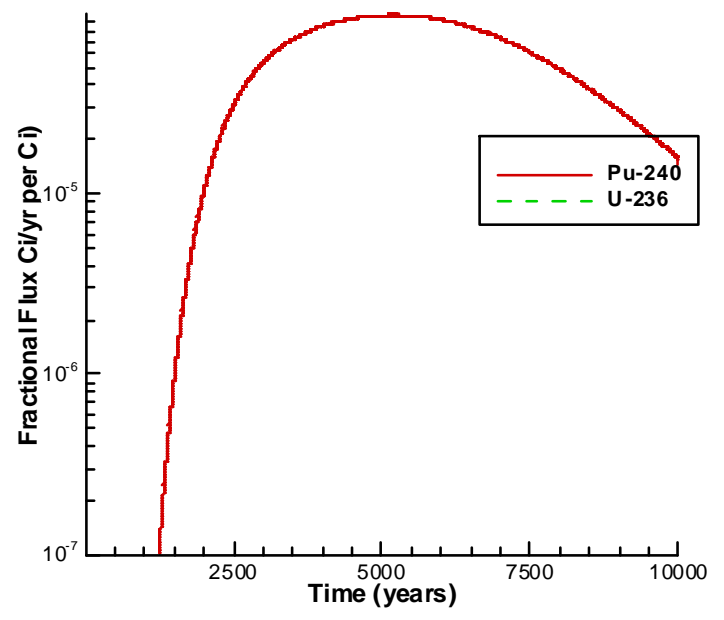

Figure CIG 50. Fluxes for Pu-240 
WSRC-TR-2003-00318

Rev. 0

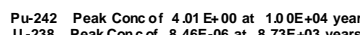

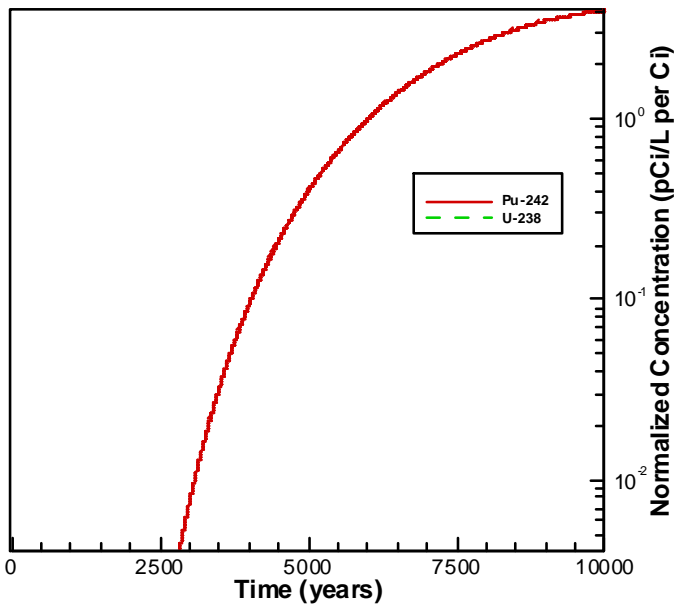

Figure CIG 51. Concentrations for Pu-242 Pu-242
U-238 Peak Flux of $1.73 E-04$ at $5.777+03$ years
Peak Fux of $1.40 \mathrm{E}-10$ at
$5.51 \mathrm{E}+03$ years

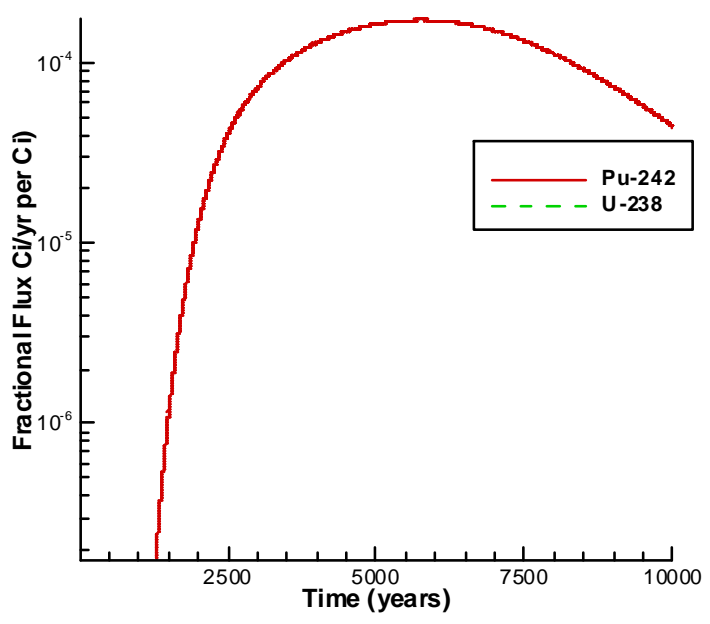

Figure CIG 53. Fluxes for Pu-242
Am-2 43 Peak Conc of $1.00 \mathrm{E}-11$ at $1.00 \mathrm{E}+04$ years $\mathrm{Np}-239$
$\mathrm{Put}-239$

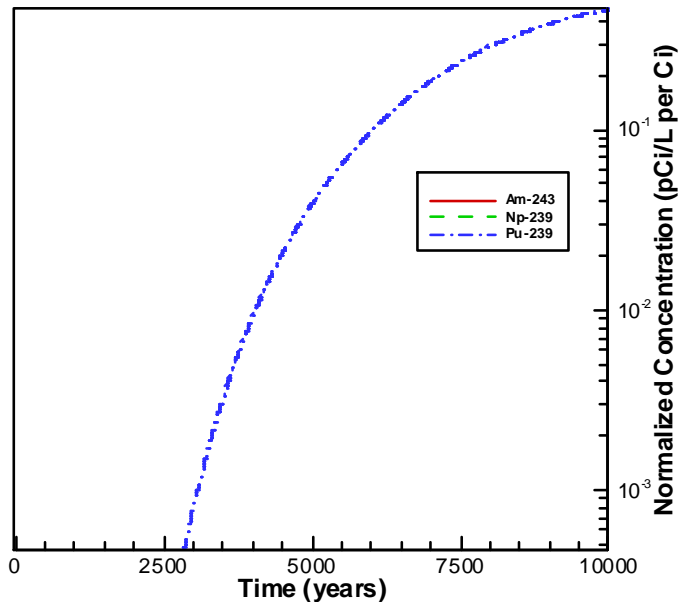

Figure CIG 52. Concentrations for Am-243 Am-2 23 Peak Flux of $1.90 \mathrm{E}-10$ at $1.00 \mathrm{E}+04$ years
$\mathrm{Np}-239$ Peak Fux of $6.85 \mathrm{E}-08$ at $1.00 \mathrm{E}+04$ years
Put-239 Peak Flux of $2.35 \mathrm{E}-05$ at $8.02 \mathrm{~B}+03$ years

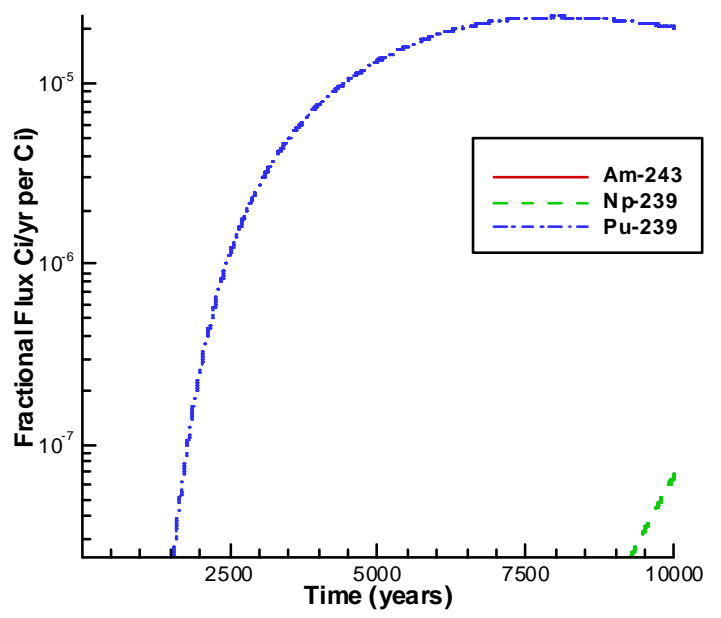

Figure CIG 54. Fluxes for Am-243 
WSRC-TR-2003-00318

Rev. 0

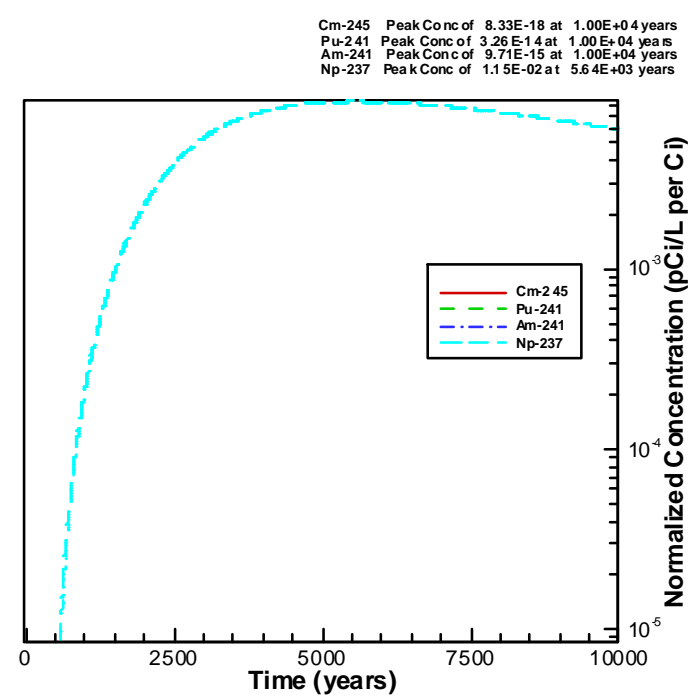

Figure CIG 55. Concentrations for Cm-245

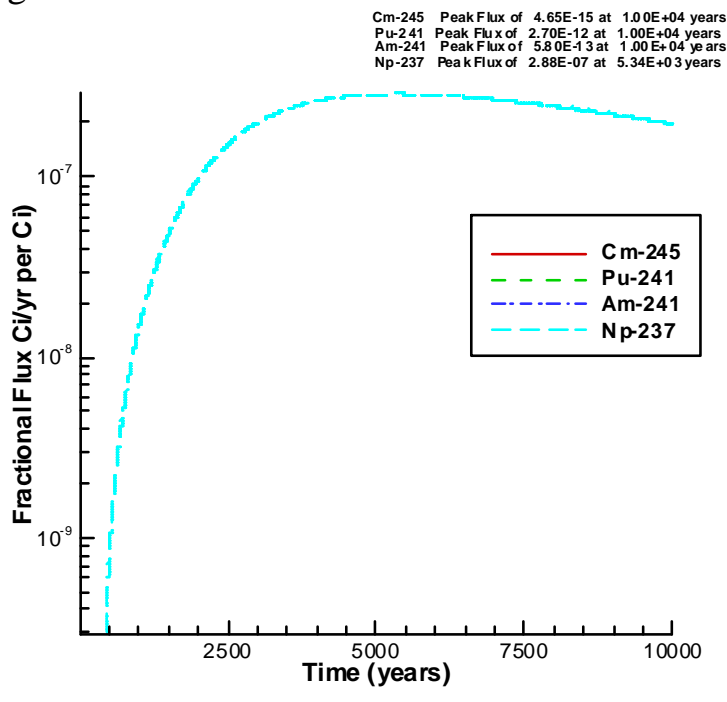

Figure CIG 57. Fluxes for Cm-245

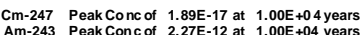
Np-239 Peak Konc of $8.50 \mathrm{E}-10$ at $1.00 \mathrm{E}+04$ years

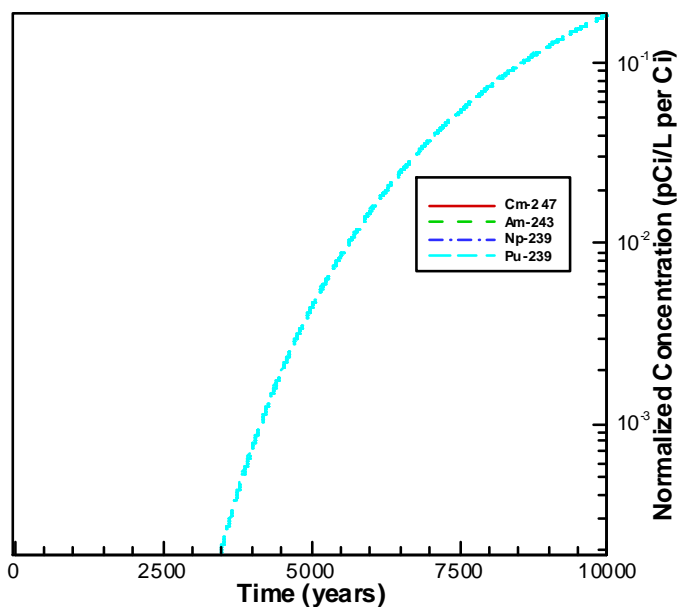

Figure CIG 56. Concentrations for Cm-247

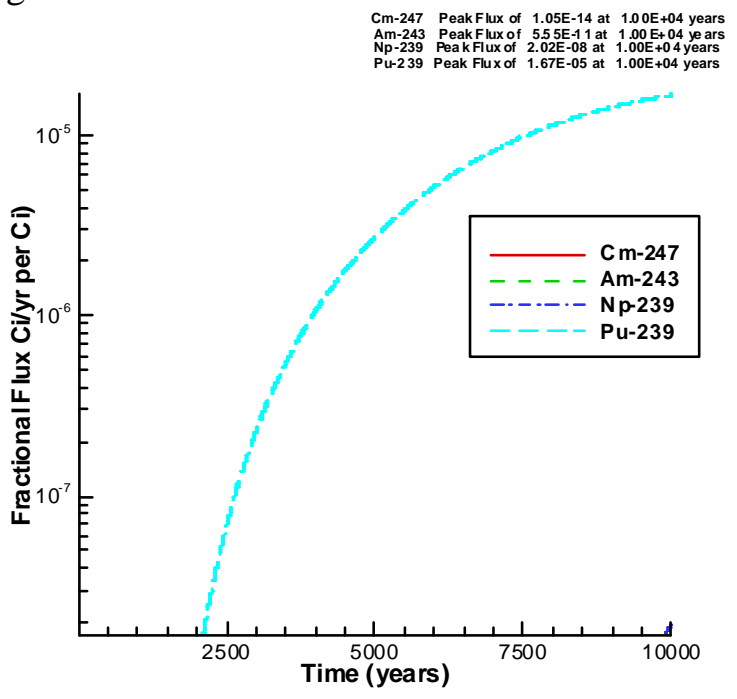

Figure CIG 58. Fluxes for Cm-247 
Cm-248 Peak Concof $1.85 E-17$ at $1.006+04$ years
Pu-244

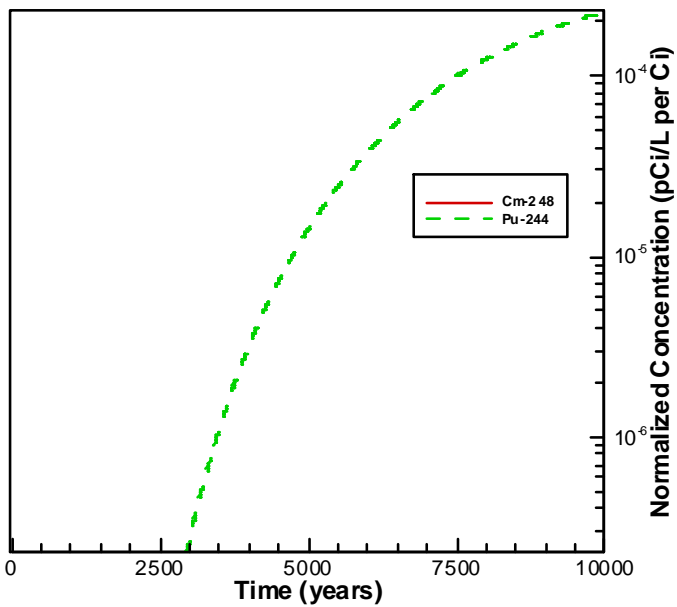

Figure CIG 59. Concentrations for Cm-248

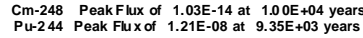

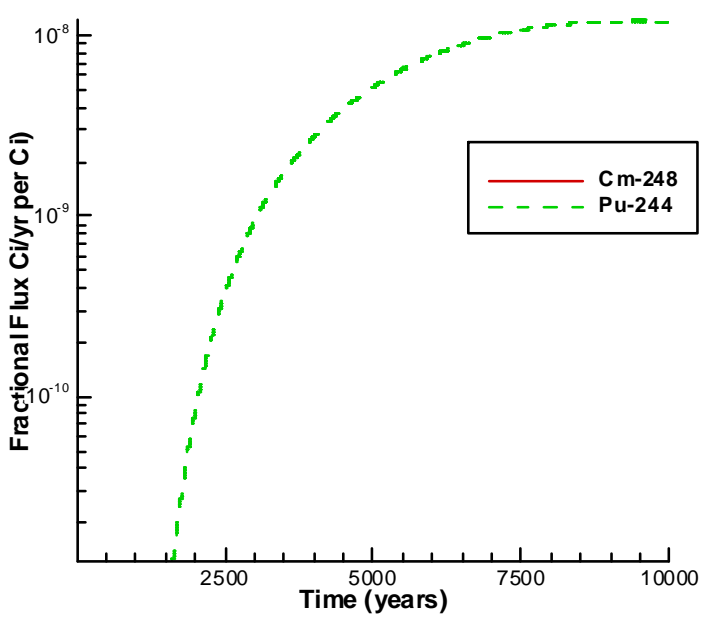

Figure CIG 61. Fluxes for Cm-248
Cf-249 Peak Conc of $3.77 \mathrm{E}-11$ at $6.99 \mathrm{E}+03$ years Pu-241 Peak Conc of $1.47 \mathrm{E}-10$ at $1.00 \mathrm{E}+04$ yea is Am-241 Peak Conc of $9.83 E-12$ at $1.00 E+04$ years
$\mathrm{Np}-237$ Peak Conc of $4.71 \mathrm{E}-04$ at $6.06 \mathrm{E}+03$ years

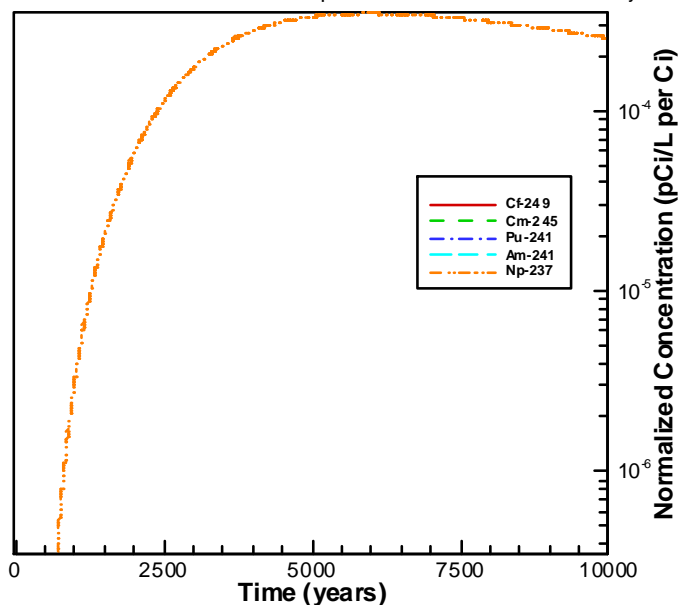

Figure CIG 60. Concentrations for Cf-249

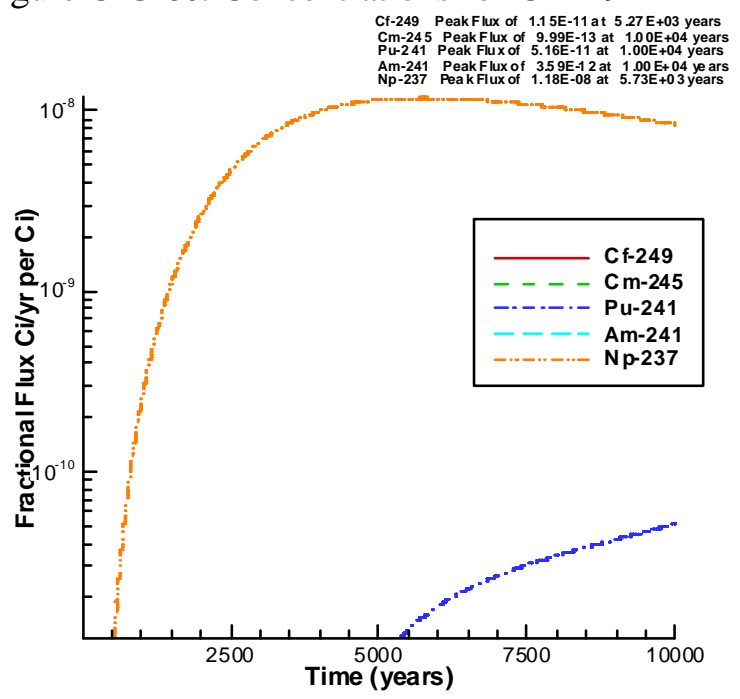

Figure CIG 62. Fluxes for Cf-249 


\section{Appendix C. Air Analysis Report}

An electronic copy of the air analysis report is included below. The headings in that report were superceded by the headings in the current report. Otherwise the contents are the same although the contents may appear on different pages than in the printed report.

Point source calculations that were performed in the air analysis report using NESHAPS approved methods were coded in CAP88. As explained below, these methods are not appropriate for an area source release where the receptor is close to the source, so different methods were developed for the area source release. If these dose estimates for an area source release are to be used for the purpose of a NESHAP permit, additional EPA approval would be required.

This air analysis report uses average meteorology. For routine dose estimates involving releases that are assumed to happen over the entire year the use of average meteorology is acceptable.

This air analysis report includes a factor of five to account for the difference between area versus point source releases. The hand calculations that were performed indicated a decrease of a factor of 7 was warranted, so decreasing the doses (from a point source) by only a factor of five provides an extra conservatism.

Scanned signature pages are included after the electronic report.

\section{WESTINGHOUSE SAVANNAH RIVER COMPANY INTER-OFFICE MEMORANDUM}

November 13, 2002

SRT-EST-2002-00184

Technical Reviewer

cc: J.B. Gladden, 773-42A

G.T. Jannik, 773-42A

P.L. Lee 773-42A

ES\&T Files, 773-42A

TO: $\quad$ L. COLLARD, 773-43A

WASTE PROCESSING TECHNOLOGY 
From: A.A. Simpkins, 773-42A (5-9643)

Environmental analysis section

\section{MODELING OF AIR RELEASES FROM THE NEW BURIAL GROUND FOR NESHAP COMPLIANCE}

As requested in your email on 10/23/2002, doses to the maximally exposed individual (MEI) located at the site boundary and at a distance of $100 \mathrm{~m}$ from the release point have been estimated for a ground level atmospheric radioactive release from the New Burial Ground, E Area. Estimates were performed for both a point source and an area source for the receptor at $100 \mathrm{~m}$. The point source estimates can be used to demonstrate compliance with the National Emission Standards for Hazardous Air Pollutants (NESHAP) (U.S. EPA 2002). The EPA computer code, CAP88 (Beres 1990), was used for this estimation. The use of CAP88 is required for demonstrating NESHAP (40CFR61) compliance. Previous dose estimates (Simpkins 1998) were not performed using the appropriate model for NESHAP and these new estimates should be used instead.

In accordance with 40CFR61, the maximally exposed individual (MEI) is assumed to be located at the nearest home, farm, business, or school and is assumed to eat vegetables, meat, and milk produced at that location. The maximally exposed individual at the site boundary is located at a distance of approximately 11,050 meters to the north. The release was assumed to be from ground level and occurred over one year. The results of the CAP88 dose estimates are shown in Table 1 for the MEI located at $100 \mathrm{~m}$ and at the boundary for unit releases $(1 \mathrm{Ci}$ ) of $\mathrm{H} 3$ and $\mathrm{C}-14$. These estimates differ from previously supplied estimates, which used MAXDOSE-SR (Simpkins 1999), primarily due to the difference in how the models utilize the meteorological data.

CAP88 has the ability to handle area sources, but the model is not deemed to be appropriate close to the source as stated in Moore et al. (1979): ' . . caution should be exercised when applying the area-source treatment where the ratio of the distance from the center (to the receptor) to the diameter of the source is less than 1.3." For the large area considered here (196 $\mathrm{m}$ by $44 \mathrm{~m}$ ), the methodology within CAP88 for handling area sources will not be used.

Although it deviates from the NESHAP model, hand calculations of air concentrations were performed for a point versus area source for average meteorological conditions, and the ratio of these two can be used as a rough approximation as to how much the dose would decrease due to the area release. This approach is approximate in that average meteorological conditions were assumed rather than the actual joint frequency distribution that is used within the CAP88 model. More detailed analysis could be performed using the actual joint frequency distribution, if desired. 
For a point source, the sector-average relative air concentration is estimated using the following Gaussian plume equation (U.S. NRC 1977):

$$
\frac{\chi}{\mathrm{Q}}=\frac{2.032}{\sigma_{\mathrm{z}} \mathrm{xU}} \exp \left[-\frac{1}{2}\left(\frac{\mathrm{H}}{\sigma_{\mathrm{z}}}\right)^{2}\right]
$$

Where

$\begin{array}{ll}\chi / \mathrm{Q} & \text { sector-average relative air concentration }\left(\mathrm{s} / \mathrm{m}^{3}\right) \\ \sigma_{\mathrm{z}} & \text { vertical diffusion coefficient }(\mathrm{m}) \\ \mathrm{x} & \text { wownwind distance }(\mathrm{m}) \\ \mathrm{U} & \text { wind speed at the release height }(\mathrm{m} / \mathrm{s}) \\ \mathrm{H} & \text { height of the release }(\mathrm{m})\end{array}$

Assuming average meteorological conditions (D stability, $4.5 \mathrm{~m} / \mathrm{s}$ windspeed) (U.S. DOE 1997) and a ground level release, the air concentration can be estimated. The vertical diffusion coefficient at $100 \mathrm{~m}$ is estimated using Pasquill Briggs Diffusion coefficients (Moore et al. 1979) as follows:

$\sigma_{\mathrm{z}}=0.06 \mathrm{x}(1+0.0015 \mathrm{x})^{-0.5}=0.06(100)[1+0.0015(100)]^{-0.5}=5.6 \mathrm{~m}$

The sector-average concentration for a point source associated with this average weather conditions would then be

$$
\frac{\chi}{\mathrm{Q}}=\frac{2.032}{\sigma_{\mathrm{z}} \mathrm{xU}} \exp \left[-\frac{1}{2}\left(\frac{\mathrm{H}}{\sigma_{\mathrm{z}}}\right)^{2}\right]=\frac{2.032}{5.6 * 100 * 4.5} \exp \left[-\frac{1}{2}\left(\frac{0}{5.6}\right)^{2}\right]=8.1 \mathrm{E}-04 \mathrm{~s} / \mathrm{m}^{3}
$$

For an area source that is square with length $2 \mathrm{a}$ with sides parallel and perpendicular to the wind direction, the sector-average concentration at $100 \mathrm{~m}$ can be estimated by (Napier 2002):

$$
\frac{\chi}{\mathrm{Q}}=\int_{-\mathrm{a}}^{\mathrm{a}} \frac{1}{\operatorname{area} * \sqrt{2 \pi} \sigma_{\mathrm{z}}(\mathrm{r}-\zeta) \mathrm{U}} \mathrm{G}(\mathrm{z}, \zeta) \mathrm{d} \zeta
$$


where

area area of the release $\left(\mathrm{m}^{2}\right)$

$\sigma_{\mathrm{z}}(\mathrm{r}-\zeta)$ vertical diffusion coefficient at distance $\mathrm{r}-\zeta(\mathrm{m})$

$\mathrm{r} \quad$ distance from the center of the release - note for $100 \mathrm{~m}$ from the edge of the contaminated area this number is $100+\mathrm{a}(\mathrm{m})$

$\zeta \quad$ variable of integration $(\mathrm{m})$

$\mathrm{G}(\mathrm{z}, \zeta)$ vertical factor which is 1 for this case since the release is ground level

$\mathrm{Z} \quad$ vertical distance of the release above ground (m)

All other terms have been previously defined.

This equation can be integrated using numerical integration such as Simpson's Rule (Beyer 1981). The dimension of the release is $196 \mathrm{~m}$ by $44 \mathrm{~m}$. Using conservation of area, this area is converted to a square with dimensions of $93 \mathrm{~m}$ by $93 \mathrm{~m}$. Numerical integration of this equation leads to a concentration estimate of $1.2 \mathrm{E}-04 \mathrm{~s} / \mathrm{m}^{3}$ that is roughly a factor of 7 less than the point source estimate of sector-average concentration of $8.1 \mathrm{E}-4 \mathrm{~s} / \mathrm{m}^{3}$ calculated above. Using this estimate, dose-release factors at $100 \mathrm{~m}$ were conservatively reduced by a factor of five to account for an area source as shown in Table 1. This conservatism is included to account for the fact that actual meteorological data was not used. The use of average meteorology is an assumption and estimates could be refined using actual meteorological joint frequency distribution data.

Methods such as these are gross approximations of atmospheric releases and should be treated as such. This methodology for an area source deviates from the approved NESHAP model and therefore, additional approval may be required before using it.

Table 1. Dose-Release Factors for Atmospheric Releases from New Burial Ground

\begin{tabular}{|l|c|c|c|}
\hline Radionuclide & $\begin{array}{c}\text { CAP88 } \\
\text { MEI at } 100 \mathrm{~m} \\
\text { Pt. Source } \\
(\mathrm{mrem} / \mathrm{Ci})\end{array}$ & $\begin{array}{c}\text { Hand-Calc } \\
\text { MEI at } 100 \mathrm{~m} \\
\text { Area Source } \\
(\mathrm{mrem} / \mathrm{Ci})\end{array}$ & $\begin{array}{c}\text { CAP88 } \\
\text { MEI at Boundary } \\
\text { Pt. Source } \\
(\mathrm{mrem} / \mathrm{Ci})\end{array}$ \\
\hline $\mathrm{H}-3$ & $7.3 \mathrm{E}-03$ & $1.5 \mathrm{E}-03$ & $2.3 \mathrm{E}-06$ \\
$\mathrm{C}-14$ & $3.5 \mathrm{E}-01$ & $7.0 \mathrm{E}-02$ & $1.1 \mathrm{E}-04$ \\
\hline
\end{tabular}




\section{$\underline{\text { Scanned Signature Pages }}$}

WSRC-IM-2002-00011

Technical Report Design Check Guidelines

SRTC Waste Treatment Technology

Figure 2

Figure 2

TECHNICAL REPORT DESIGN CHECKLIST

Report Tite: Motelung of Relecoses from the

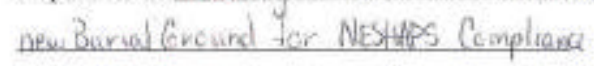
Document No. SRT-EST-2002-60184 Author(s): Ali A. Simpkins
Reviewer: Patricic Lee

Activity Code: $L 0410$

Current Date: $11 / 7 / 02$

Date Needed: $11 / 13 / 02$

Location of report and supplemental information:

supplied at sent uia email

Analytical/Experimental Instruction: Revifut methods to enswe Ligical

Approach

Mathematical Check Instruction: Spol Check Spreadsheet

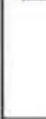
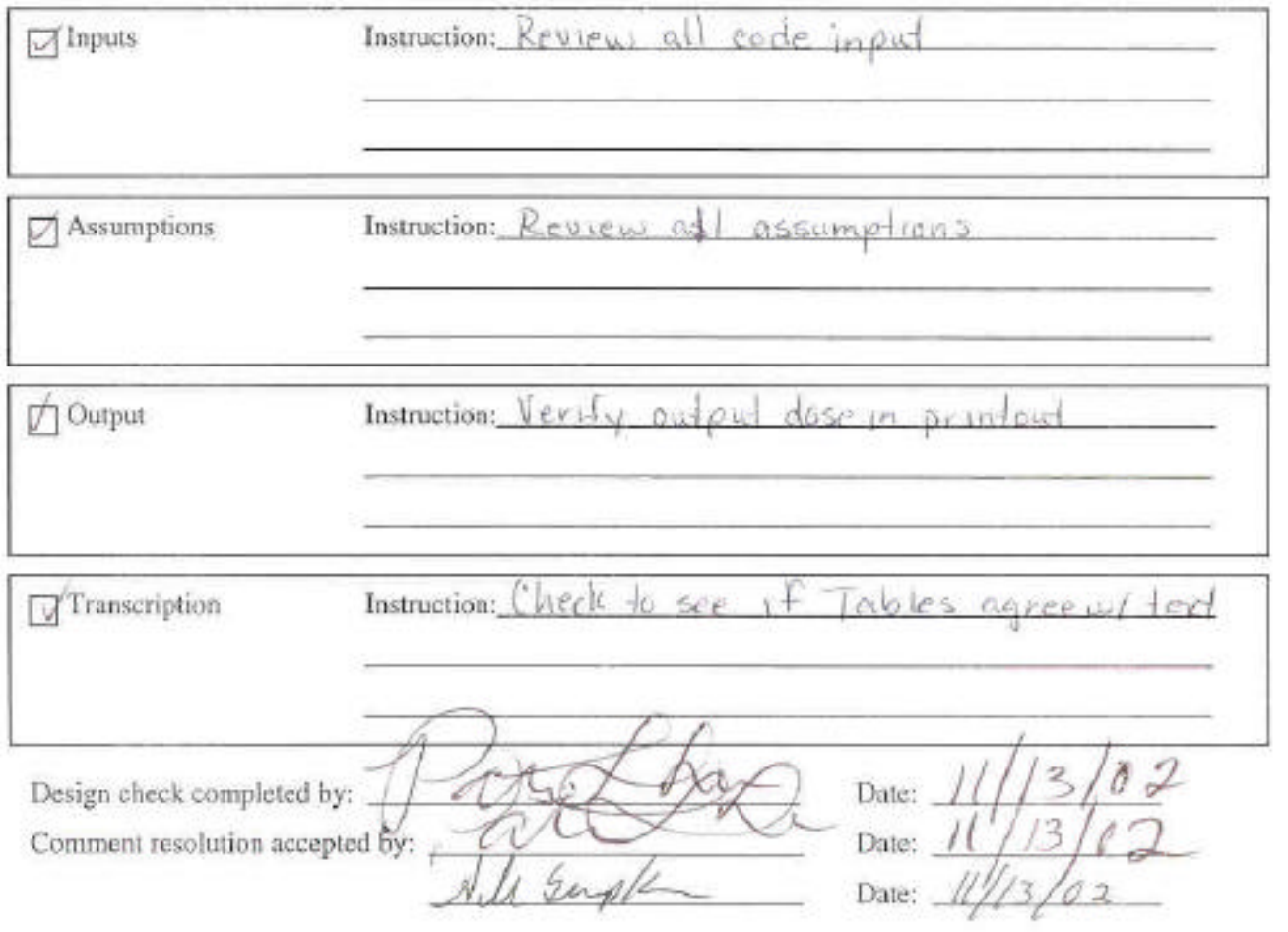


\title{
WESTINGHOUSE SAVANNAH RIVER COMPANY
}

\section{INTER-OFFICE MEMORANDUM}

November 13,2002

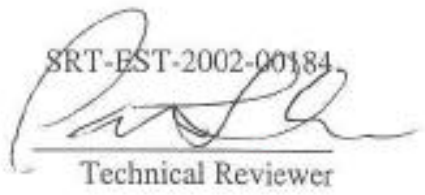

cc: J.B. Gladden, $773-42$ A

G.T. Jannik, $773-42 \mathrm{~A}$

P.L. Lee 773-42A

ES\&T Files, 773-42A

\author{
TO: $\quad$ L. COLLARD, 773-43A \\ WASTE PROCESSING TECHNOLOGY \\ A. 15 \\ FROM: A.A. SIMPKINS, 773-42A (5-9643) \\ ENVIRONMENTAL ANALYSIS SECTION
}

\section{MODELING OF AIR RELEASES FROM THE NEW BURIAL GROUND FOR NESHAP COMPLIANCE}

As requested in your email on 10/23/2002, doses to the maximally exposed individual (MEI) located at the site boundary and at a distance of $100 \mathrm{~m}$ from the release point have been estimated for a ground level atmospheric radioactive release from the New Burial Ground, E Area. Estimates were performed for both a point source and an area source for the receptor at $100 \mathrm{~m}$. The point source estimates can be used to demonstrate compliance with the National Emission Standards for Hazardous Air Pollutants (NESHAP) (U.S. EPA 2002). The EPA computer code, CAP88 (Beres 1990), was used for this estimation. The use of CAP88 is required for demonstrating NESHAP (40CFR61) compliance. Previous dose estimates (Simpkins 1998) were not performed using the appropriate model for NESHAP and these new estimates should be used instead.

In accordance with 40CFR61, the maximally exposed individual (MEI) is assumed to be located at the nearest home, farm, business, or school and is assumed to cat vegetables, meat, and milk produced at that location. The maximally exposed individual at the site boundary is located at a distance of approximately 11,050 meters to the north. The release was assumed to be from ground level and occurred over one year. The results of the CAP88 dose estimates are shown in Table 1 for the MEI located at $100 \mathrm{~m}$ and at the boundary for unit releases $(1 \mathrm{Ci})$ of $\mathrm{H}-3$ and $\mathrm{C}-14$. These estimates differ from previously supplied estimates, which used MAXDOSE-SR (Simpkins 1999), primarily due to the difference in how the models utilize the meteorological data. 


\section{Appendix D. Groundwater Pathway Model QA Changes}

The solubility-limited models for U-238 and $\mathrm{Pu}-239$ incorporate a new Porflow computer command, FIXED, that was not previously tested. A simple test case was developed to ensure that the command functioned correctly. Conceptually, a two-dimensional model was developed with a length of 10 feet and a height of 1 foot see Figure 3). Flow was restricted to move in the positive X-direction at $10 \mathrm{ft} /$ year. The porosity was set at 0.5 and the domain was fully saturated. A concentration of $1 \mathrm{Ci} / \mathrm{cu} \mathrm{ft}$ was fixed in the two center cells. No flux was allowed in the Y-direction and at both $\mathrm{X}$-boundaries the concentrations were set at zero $\mathrm{Ci} / \mathrm{cu} \mathrm{ft}$.

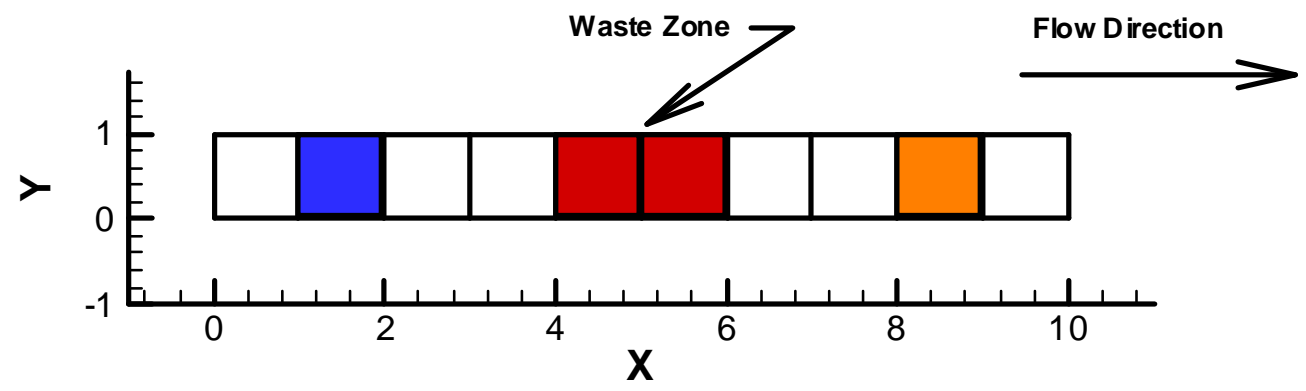

Figure 3. Conceptual model for fixed concentration test case

Concentration histories over a 1000 year period were reported at the two center cells, one upstream cell, and one downstream cell (see Figure 4). The two waste cells showed constant $1 \mathrm{Ci}$ per cubic foot concentrations throughout the analysis. The concentration in the downstream cell quickly increased to $1 \mathrm{Ci} / \mathrm{cu} \mathrm{ft}$ where it remained. The concentration in the upstream cell remained at $0 \mathrm{Ci} / \mathrm{cu} \mathrm{ft}$ throughout the analysis. These results demonstrate that the FIXED command functioned correctly. The Porflow input file is provided below.

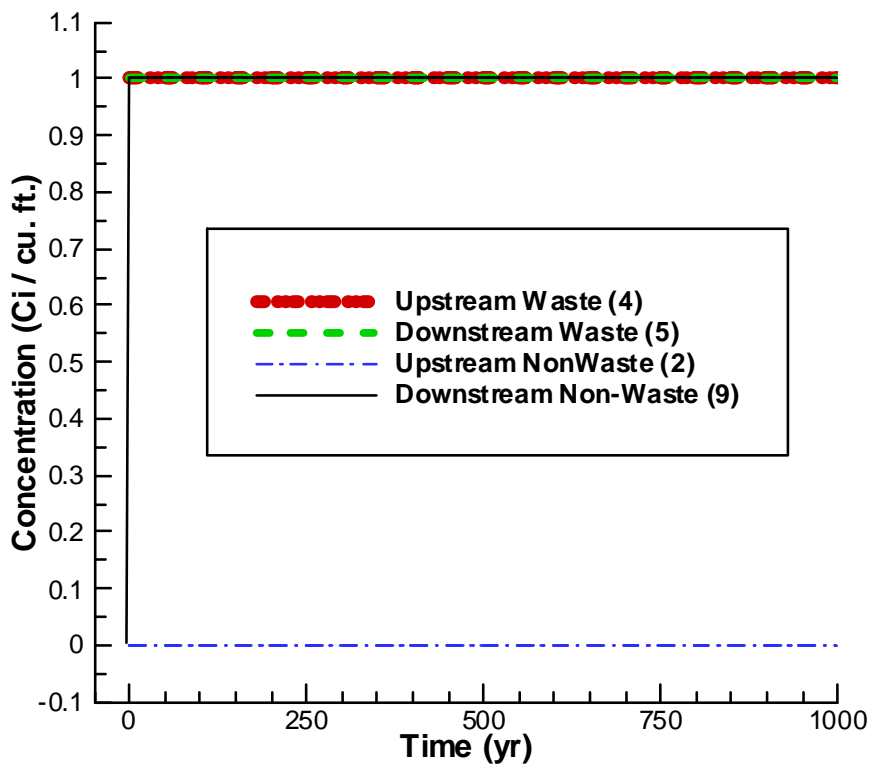

Figure 4 Concentration histories for fixed concentration test case 
TITLE Test FIXED command Transport

GRID 12 by 3

COOR X 012345678910

COOR Y 01

MATErial type 1 from $1 \quad 1$ to 123

MATErial DENSity 2.65

MATErial POROsity $=.5 .5$

TRAN for $\mathrm{C} \mathrm{Kd}=0$ diff $=0$ al $=0$ at $=0$

LOCA $(1,1)$ to $(12,3)$ ID=DOMA

LOCA $(4,1)$ to $(5,3)$ ID=WAST

BOUN C X-valu $=0$.

BOUN C X+valu=0.

BOUN C Y- flux $=0$.

BOUN C Y+FLUX $=0$.

FIXED C 1 ID=WAST

SET $U=10$

SET $S=1$

PROPerty for $\mathrm{C}$ is HARMonic

MATRix in $\mathrm{X}$ and $\mathrm{Y}$ for $\mathrm{C}$ in 3 sweep using $\mathrm{ADI}$

DIAG TIME U S C at (4,2) every 1000 steps

OUTPut every 900000 steps

CONVergence for C REFE GLOBal 1.e-3, max iterations $=30$

DISAble FLOW

HIST AT 4,2 5,2 2,2 9,2

HIST C TIME 'run.his' 1 yr

FLUX C ID=DOMA 'RUN.FLX' TIME 1.0E+00 yr

FLUX C ID=WAST 'RUN.FLX' TIME 1.0E+00 yr

SOLV C $1000 \mathrm{dt}$ 1.0E-04 inc 1.001 max 1

SAVE U V S C in 'END1k.ARC' NOW

END 


\section{Appendix E. Design Check}

\section{E.1 Design Check for Groundwater Pathway}

A two-part design check was completed for the groundwater pathway analyses. The initial design check noted that an incorrect porosity was used for the waste zone in the vadose zone models. That error was corrected and rechecked. The design check instructions and report are provided in this section.

\section{E.1.1 Design check instructions for groundwater pathway}

Perform a design check for Special Analysis for Disposal of Cement-Stabilized Encapsulated Waste at the E-Area Low-Level Waste Facility, WSRC-RP_99-00596, Rev 1, May 22, 2003 following the general guidance provided in WSRC-IM-2002-00011.

\section{E.1.1.1 Original Request}

1. A. (for Elmer)

- Check the APPROACH to ensure that the conceptual models are reasonable, appropriate, and consistent with the PA. Special areas to focus on are

- Does the screening process remove nuclides that should not be removed

- Does the vadose zone model represent field operations

1. B. (for Thong)

- Check the APPROACH to ensure that the conceptual models are reasonable, appropriate, and consistent with the PA. Special areas to focus on are

- Are there any conditions where the aquifer model with a reduced footprint would produce answers that are different from the full aquifer model

2. Check the MATHEMATICS by

- Spot checking mass balance information produced by Porflow

- Check that Pu-241 and Am-241 results are correctly calculated in the flux and concentration tables

3. Check to ensure that the INPUTS are correct for

- One nuclide with no progeny and one nuclide with at least 2 progeny

Input checks include flow and transport files for vadose zone and aquifer models

- Check that the modeling changes stated in the report were properly implemented

4. Check to ensure that the OUTPUTS are reasonable by

- For One nuclide with no progeny and one nuclide with at least 2 progeny

Check the fractional flux data that is plotted

(note that the unprocessed output is for an inventory of $1 \mathrm{E} 6 \mathrm{Ci}$, thus the fractional fluxes are

divided by 1E6) 
Check the fractional flux data that is uses as a source file

Check the well concentrations

(note that the unprocessed output is in units of $\mathrm{Ci} / \mathrm{ft}^{3}$, while processed data are in units of

$\mathrm{pCi} / \mathrm{L})$

- For each nuclide, check that the general pattern well concentrations "matches" the general pattern of the fluxes

- Check the concentration patterns among nuclides versus their Kds and half-lives for anomalies

5. TRANSCRIPTION:

- Ensure that the peak concentrations, peak fluxes and times in the figures match the values in the tables. If the numbers are presented at two different locations throughout the report, they should be identical, except for rounding.

\section{E.1.1.2 New Request}

Changes for Thong after authors revised report based on initial comments

1. Check that initial errors detected have been corrected

2. Complete any items on design check list not previously performed

3. Check the MATHEMATICS by

- Spot checking mass balance information produced by Porflow

- Check that for chains with Pu-241, Am-241, Cm-242, and Bd-249 as parents, results are correctly calculated in the flux and concentration tables

4. Check U-238 and Pu-239 values in Table 5.1-13, because they were modeled differently, as solubility-limited

5. Check concentration limits in Table 5.1-13

\section{E.1.2 Design Check Report}

\section{E.1.2.1 Original Request}

\section{$\underline{\text { Item 1A }}$}

The conceptual models are reasonable and appropriate. They improve on those used in the PA by more accurately representing the disposal of waste encapsulated in grout. The screening method (i.e., use of the slit trench screening results) does not inappropriately remove radionuclides. The vadose zone model adequately represents field operations. 


\section{Item 1B}

Aquifer model with reduced footprint (Cut model) should produce same answers as the full aquifer model if same flow field and correct sources are used.

---> Big model (Run.dat) and reduced model (CutRun.dat) were checked.

\section{$\underline{\text { Item } 2}$}

- Checked all nuclides input files for correctly reading flow field and $\mathrm{Kd}$ files: OK

- Mass balance: Spot checked mass balance for Aquifer/Transport, Vadose/Transport/BadCap and Vadose/Transport/OKCap. The results show disparity less than $1 \%$.

Model

Aquifer/Transport

Aquifer/Transport

Aquifer/Transport

Aquifer/Transport

Aquifer/Transport
Nuclide

C-14

$\mathrm{Ni}-59$

Sr-90

Tc-99

U-235

Pa-231

Ac-227

Th-227

Ra-223

Nuclide

Vadose/Transport/OKCap

Vadose/Transport/OKCap

Vadose/Transport/OKCap

Vadose/Transport/OKCap

Vadose/Transport/OKCap

Vadose/Transport/BadCap

Vadose/Transport/BadCap

Vadose/Transport/BadCap

Vadose/Transport/BadCap

Vadose/Transport/BadCap
C-14

$\mathrm{H}-3$

Pu-239

U-235

Tc-99

$\mathrm{Zr}-93$

$\mathrm{Nb}-$

$93 \mathrm{~m}$

Nuclide

C-14

I-129

$\mathrm{Ni}-59$

Tc-99

U-235

$\mathrm{Pa}-231$

Ac-227

Cumulative Total Inflow
$7.878594 \mathrm{E}-01$
$5.247530 \mathrm{E}-01$
$1.180888 \mathrm{E}-05$
$9.988839 \mathrm{E}-01$
$5.897845 \mathrm{E}-01$
$3.651007 \mathrm{E}-02$
$3.250339 \mathrm{E}+00$
$1.366633 \mathrm{E}+03$
$2.241235 \mathrm{E}+03$

Total Initial Property or Current Total

Property $1.000000 \mathrm{E}+06$ $1.000000 \mathrm{E}+06$ 2.283012E-06 $5.322360 \mathrm{E}-08$ $1.000000 \mathrm{E}+06$ $1.000000 \mathrm{E}+06$

$9.966685 \mathrm{E}+05$

\section{Total Initial Property} or Current Total

Property $9.643604 \mathrm{E}+05$ $9.999879 \mathrm{E}+05$ $9.974041 \mathrm{E}+05$ $9.990149 \mathrm{E}+05$ 9.999997E+05 1.327693E+05 $1.330274 \mathrm{E}+05$
Cumulative Flux

Disparity 8.402502E-14 6.674291E-07 $-2.761629 \mathrm{E}-14$

6.772360E-15 1.643324E-12 2.040035E-15 $-4.975187 \mathrm{E}-15$ $-4.133846 \mathrm{E}-13$ 1.996799E-12

Cumulative Flux Disparity -1.605534E-05

$-4.119329 \mathrm{E}-03$ 1.969871E-08 $-6.599450 \mathrm{E}-13$ 9.512900E-03 1.452264E-09

6.753253E-07

Cumulative Flux

Disparity 3.038763E-01

$-7.400499 \mathrm{E}-01$

$-2.346743 \mathrm{E}+00$

$-6.148256 \mathrm{E}-01$ $-8.192147 \mathrm{E}-02$ $-7.610163 \mathrm{E}-02$ $-8.624210 \mathrm{E}-02$
Disparity \% 0.00 0.00 0.00

0.00

0.00

0.00

0.00

0.00

0.00

Disparity \%

0.00

0.00

0.86

0.00

0.00

0.00

0.00

Disparity \%

0.00

0.00

0.00

0.00

0.00

0.00

0.00 
- Check Pu-241, Am-241, Cm-242, and Bk-249 for correct calculation of flux (Table 4.37) and concentration (Table 5.1-7). The calculated values as shown below agree with those given in tables.

\section{$\mathrm{T}_{1 / 2}(\mathrm{yrs})$}

$\begin{array}{ll}\text { Pu-241 } & 14.4 \\ \text { Am-241 } & 432 \\ \text { Cm-242 } & 0.45 \\ \text { Bk-249 } & 0.88 \\ \text { Np-237 } & 2.14 \text { E6 } \\ \text { Cf-249 } & 350 \\ \text { U-234: } & 2.45 E 5\end{array}$

Flux (Table 4.3-7):

Np-237 (parent): $\quad 3.08 \mathrm{E}-4 \mathrm{Ci} / \mathrm{yr}$

Np-237 (daughter of Pu-241): (3.08E-4 Ci/yr) (14.4/2.14E6) = 2.0725E-9 Ci/yr

Np-237 (daughter of Am-241): (3.08E-4 Ci/yr) (432/2.14E6) = 6.2175E-8 Ci/yr

U-234 (parent): $\quad$ 7.19E-5 Ci/yr

U-234 (daughter of Cm-242): (7.19E-5 Ci/yr) $(0.45 / 2.45 \mathrm{E} 5)=1.32 \mathrm{E}-10 \mathrm{Ci} / \mathrm{yr}$

Cf-249 (parent): $\quad 1.15 \mathrm{E}-11 \mathrm{Ci} / \mathrm{yr}$

Cf-249 (daughter of Bk-249): $(1.15 \mathrm{E}-11 \mathrm{Ci} / \mathrm{yr})(0.88 / 350)=2.89 \mathrm{E}-14 \mathrm{Ci} / \mathrm{yr}$

Concentration (Table 5.1-7):

$\mathrm{Np}-237$ (parent): $\quad 1.22 \mathrm{E}+1 \mathrm{pCi} / \mathrm{L}$

Np-237 (daughter of Pu-241): (1.22E+1 pCi/L) $(14.4 / 2.14 \mathrm{E} 6)=8.21 \mathrm{E}-5 \mathrm{pCi} / \mathrm{L}$

$\mathrm{Np}-237$ (daughter of Am-241): (1.22E+1 pCi/L) $(432 / 2.14 \mathrm{E} 6)=2.46 \mathrm{E}-3 \mathrm{pCi} / \mathrm{L}$

U-234 (parent): $\quad 2.83 \mathrm{pCi} / \mathrm{L}$

U-234 (daughter of Cm-242): $(2.83 \mathrm{pCi} / \mathrm{L})(0.45 / 2.45 \mathrm{E} 5)=5.2 \mathrm{E}-6 \mathrm{pCi} / \mathrm{L}$

Cf-249 (parent): $\quad 3.77 \mathrm{E}-11 \mathrm{pCi} / \mathrm{L}$ 
Cf-249 (daughter of Bk-249): (3.77E-11 pCi/L) $(0.88 / 350)=9.48 \mathrm{E}-14$ pCi/L

\section{$\underline{\text { Item } 3}$}

Input files (RUN.DAT) for the following were checked:

1) Aquifer/Transport (for Tc-99, and Th-232 with daughters Ra-228, Th-228, Ra224)

2) VadoseZ/Flow/OKCap and VadoseZ/Flow/BadCap

3) Vadose/Transport/OKCap (for C-14 K\&L Basin, and Am-243 with daughters Np239, Pu-239) and Vadose/Transport/BadCap (for C-14 K\&L Basin, and Cm-245 with daughters $\mathrm{Pu}-241, \mathrm{Am}-241, \mathrm{~Np}-237$ )

Inputs (including porosity data) are correctly set up to incorporate all modeling changes stated under Sections 3.1 and 3.2

\section{$\underline{\text { Item } 4}$}

- Fractional flux data for Ni59 were checked. Two methods were used to obtain fractional flux:

1. Use of ?(total cumulative outflow)/?t

2. Use of total instantaneous influx.

Method 1 shows a peak flux of $1.7527 \mathrm{E}-4$ at 8545 years. In method 2, the peak flux of $1.7523 \mathrm{E}-4$ is at 8509 years. The report, in which method 1 was used, shows a peak flux of $1.753 \mathrm{E}-4$ at 8545 years. It was also noted that method 2 gives a smooth fractional flux curve over time, while method 1 shows that data bounce around the peak flux.

- Flux data for U-238 with daughters Th-234 and U-234 were checked. Again the two methods as described above were used to obtain fractional flux. Below are the results obtained:

\begin{tabular}{lrrrrrr} 
& \multicolumn{2}{c}{ Method 1 } & \multicolumn{2}{c}{ Method 2 } & \multicolumn{2}{c}{ Report } \\
& Time & Peak & Time & Peak & Time & Peak \\
& (yrs) & Flux & (yrs) & Flux & (yrs) & Flux \\
& & $1.06 E-$ & & $1.06 E-$ & & $1.06 E-$ \\
U-238 & 8534 & 12 & 9962 & 12 & 8623 & 12 \\
Th- & & $1.18 E-$ & & $1.16 E-$ & & $1.18 E-$ \\
234 & 10000 & 14 & 9948 & 14 & 10000 & 14 \\
& & $1.96 E-$ & & $1.92 E-$ & & $1.96 E-$ \\
U-234 & 10000 & 08 & 9993 & 08 & 10000 & 08
\end{tabular}


Method 1 gives the same results as those in the report except the time at which U-238 flux peaks.

- The fractional flux data used as source file (SOUR.DAT) were spot checked for Ni59 and U-238. Flux data from SOUR.DAT are plotted together with those fluxes obtained from method 1. The plots show identical curves.

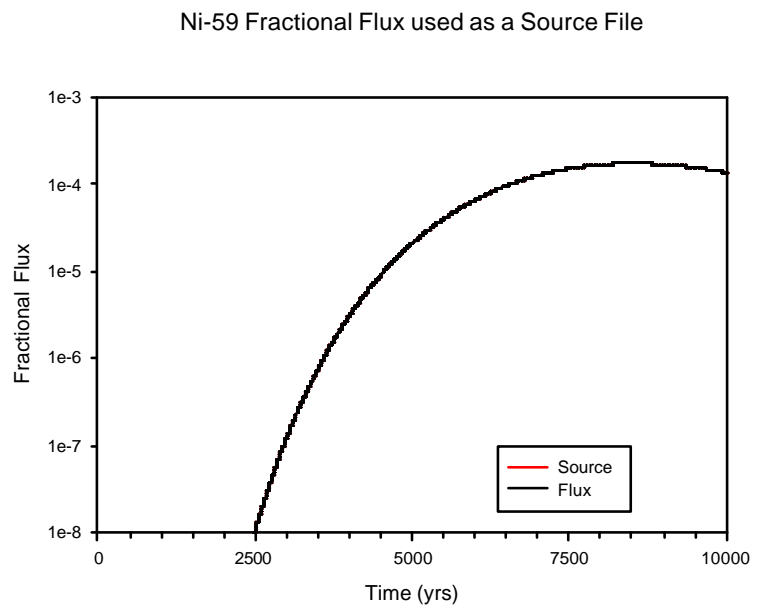

U-238 Flux used as a Source File

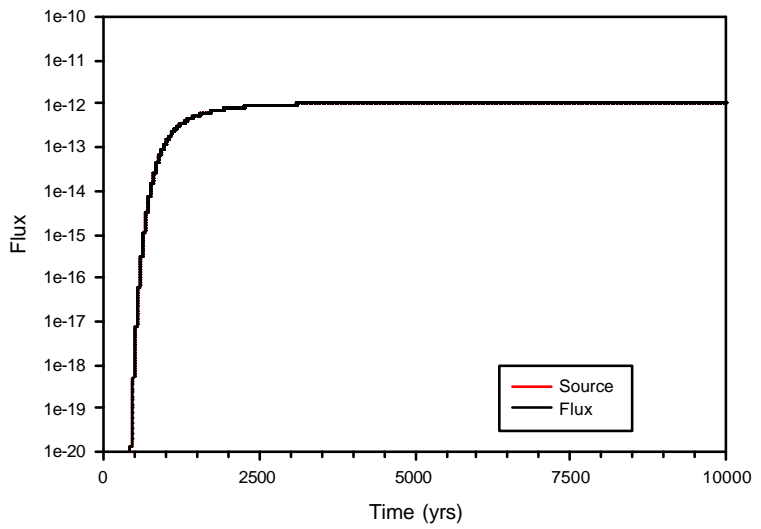

- Well concentrations check for I-129 and U-232 (daughters Th-228 and Ra-224). For I229 , concentration curve, the peak concentration and the peak time agree with those in the report. For U-232, the concentration curves, peak concentrations and peak times don't agree with those in the report. 

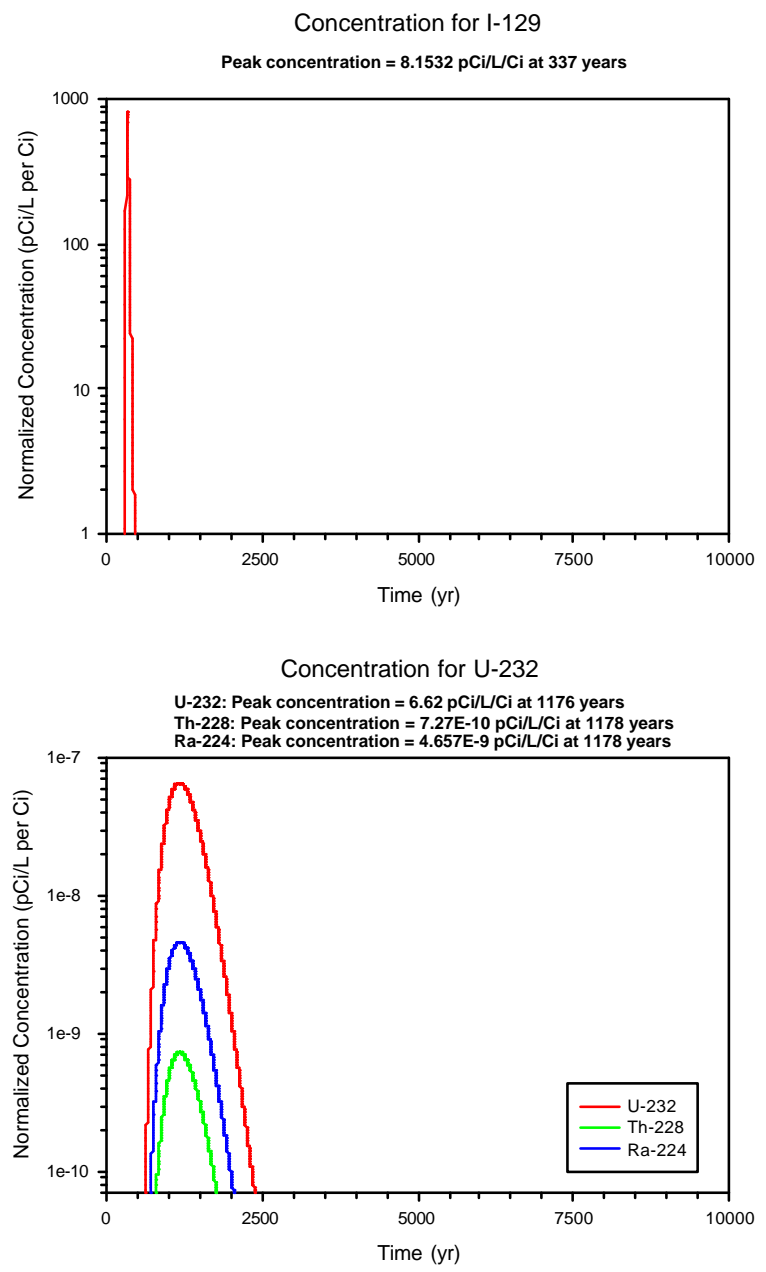

- For each radionuclide, check that the general pattern of well concentrations "matches" the general pattern of the fluxes:

- For U-235, daughters Pa-231, Ac-227, and Ra-223 display suspicious pattern of the fluxes.

- Pu-238 curves are missing in Figure CIG 44 and Figure CIG 46.

○ U-236 curves are missing in Figure CIG 48 and Figure CIG 50.

- U-238 curves are missing in Figure CIG 51 and Figure CIG 53.

- Only Pu-239 curves are shown in Figure CIG 52 and Am-243 curves are missing in Figure CIG 54.

○ Only Np-237 curves are shown in Figure CIG 55 and Figure CIG 57.

- Only Np-239 curves are shown in Figure CIG 56 and Figure CIG 58.

$\circ$ Cm-248 curves are missing in Figure CIG 59 and Figure CIG 61

○ Only Np-237 is shown in Figure CIG 60.

○ Cm-245, Pu-241 and Am-241 are missing in Figure CIG 62.

- Check the concentration patterns among nuclides vs. their Kds and half-lives for anomalies. Cs-135, Cm-246 and Ni59 were spot checked. No abnomalies were observed. 


\section{$\underline{\text { Transcription }}$}

- Peak concentrations, peak fluxes and peak times in the figures and in the tables were spot checked for Ni59, Tc-99, U-232 (daughters Th-228 and Ra-224), and Th-232 (daughters Ra-228, Th-228 and Ra-224). Values for Ni59 and Tc-99 are correct. But values for U$232+$ daughters and Th-232+daughters in the figures are different from those in tables.

Need to re-check all tables.

E.1.2.2

New request

\section{$\underline{\text { Item } 3}$}

- See Item 2 in the original request.

\section{$\underline{\text { Item } 4}$}

- Check U-238 and Pu-239 values in Table 5.1-13. Values are correct.

\section{$\underline{\text { Item } 5}$}

- Check concentration limits in Table 5.1-13. All values are correct. Some radionuclides don't have concentration limits listed (i.e., Pu-241 with daughter Am-241; Am-241; Cm242; and Bk-249).

\section{E.2}

\section{Intruder Pathway}

\section{Design Check for}

\section{E.2.1 Design check instructions for intruder pathway}

1. Verify the logic and correctness of the equations on the spreadsheet "UPDATE CIG INTRUDER.XLS".

2. Spot check data in columns B through $A D$ and Al for consistency with Tables 6.31 through 6.3-4 in EAV PA Rev 1.

3. Verify that the F Factors (Columns AF, AG and AH) are correctly transcribed from PORFLOW data (attached). For those F Factors that were calculated, verify the logic and execution of the calculation.

4. Verify that numbers in spreadsheet columns AL, AO and AP are correctly transcribed to the intruder result tables for Post Drilling, Resident and Agriculture scenarios, respectively. 
5. Verify that numbers in spreadsheet columns AS and AT are correctly transcribed from the tables of groundwater and air results, respectively.

6. Verify that the limits calculated in Column AU are correctly calculated in the spreadsheet and transcribed to the limits table in the report.

\section{E.2.2}

Design check report

The subject spreadsheet and tables in the report were generally found to be correct with comments, questions and corrections noted in the discussion of specific spreadsheet columns below. The approach to checking each column is shown in bold and below it observations have been itemized. Responses by the author to the design check are shown in italics below specific observations. Where appropriate, responses back to the author are underlined.

\section{A. Column B and C; Branching Fraction and Half Life. Compared values with those in Table C.3-1 in the E-Area PA Revision 1.}

1. Half life of Th-231 incorrect (see marked up spreadsheet). Other small rounding differences between spreadsheet and PA table (not generally noted).

Correction made.

2. Missing nuclides include Mo-93 and Th-230 and daughters. Please explain.

Nuclides added.

\section{B. Column D and E; Ingestion and Inhalation DCFs. Compared values with Table C.3- 2 in the E-Area PA Revision 1.}

1. DCF values for Ac-225 and $\mathrm{Bi} 213$ (Ra-225 daughters) in spreadsheet not found in PA table. Where did they come from?

They came from DOE/EH-0071, the source of the other DCFs in this spreadsheet.

DCFs from this source were spot-checked and found to be correct.

2. Where the PA table denotes daughters but does not list them it uses the notation "+d". In your spreadsheet you sometimes list these daughters and sometimes do not (see for example, "Ra-225 +d" vs. "Ra-223 +d"; also see "Np-237+d"). Please explain.

The use of the "+" in the spreadsheet is an aid to me in keeping track of short-lived daughters. It does not necessarily correspond to the notation in the PA tables. Generally, the determination on when to include the contribution from short-lived daughters in an EDE depended upon a number of factors including relative half-life, availability and magnitude of the daughter DCFs, decay rate mechanism and branching factors. 
Checking the resulting EDE from your spreadsheet against the corresponding EDE table produced the same results indicating that the logic used in establishing the PA value was followed.

C. Column F; External DCFs for nuclides uniformly distributed in $15 \mathrm{~cm}$ of soil (PA Table C.3-3), and Columns G, $H$ and I (PA Table C.3-4) for nuclides uniformly distributed in an infinite soil column. Compared values with referenced PA table.

1. DCF values for numerous nuclides in Columns $F$ and $G$ are not found in the corresponding PA table. They are noted in the marked up copy of your spreadsheet. Where did they come from?

The tables in the PA do not include data for radionuclides that turn out to be insignificant. The spreadsheets that we use contain a greater number of radionuclides. The data for the direct gamma exposure came from Dave Kocher's calculations, and he has had them thoroughly checked.

2. A number of DCFs are omitted from spreadsheet. See marked up spreadsheet for additions.

The missing values have been added.

3. Several small rounding differences in DCFs noted in Columns G and I in marked up spreadsheet.

The corrections were made.

D. Columns M and AJ; Plant to Soil Concentration Ratios and Geometric Factor, respectively. Compared values with Table C.3-6 and Table 6.3-4 in PA Revision 1, respectively.

1. PA Table and spreadsheet values were all the same. Correct Geometric Reduction Factor was employed.

No changes needed.

E. Columns K, N, P, R, T, V, X, and Z; EDEs for Drinking Water, Vegetables, Soil Ingestion, Garden Exposure, Home Exposure (Ag Scenario), Home Exposure (Res Scenario), Garden Inhalation, and Home Inhalation, respectively. Checked logic and correctness of computation and compared with Table C.3-5, 3-7, 3-8, 3-9, 3-10, 3-14, 311, and 3-12, respectively, in PA Revision 1.

1. Basic EDE equation logic was found to be correct in all cases. Results were spot checked with hand calculation and found to be implemented correctly in the spreadsheet. Several 
questions arose with respect to treatment of daughters that are discussed in the following comments

2. The rule is not clear as to when to include the EDE contributions from daughters into the parent's EDE. In some cases contributions are included while in others they are not. For example, U235 decays as follows: U235->Th231->Pa231->.... Only Th231 is included in the U235 EDE. In another example, U234 decays as follows: U234->Th230->Ra226->... None of the daughter contributions are included with the parent. This is more a question of the treatment of them in the PA revision since the spreadsheet seems to simply implement the logic of the PA. A general explanation would be sufficient.

In general, the first level of daughters are summed up in the limits columns. The second level daughters are summed to the first level daughters in the EDE columns.

3. A number of nuclides included in the spreadsheet were excluded from the subject PA tables. Most notably different was Column K when compared with Table C.3-5 (Drinking Water EDEs). These are marked on the spreadsheet for this column. Please explain.

As noted above, many radionuclides are in the spreadsheet that do not appear in the PA tables. In particular, the ones noted in this comment are radionuclides that have been screened from the groundwater analysis.

4. EDE value for $\mathrm{Zr}-93$ did not include contribution from daughter $(\mathrm{Nb}-93 \mathrm{~m})$ in columns $\mathrm{K}$ and $\mathrm{V}$, but did include it in columns $\mathrm{N}, \mathrm{P}, \mathrm{T}, \mathrm{X}$ and $\mathrm{Z}$.

Contributions from the Nb-93 daughter were added in columns $K$ and $V$.

5. Should the EDE from vegetables (column N) for Bi213 (Ra225 daughter) be zero when it has a nonzero ingestion DCF (column D)?

As noted in footnote c of Table C.3-6, this is a short-lived daughter that is not taken up by plant root independent of the parent. This is how very short-lived daughters are consistently treated in the analysis, e.g., Sr-90 - Y-90, and Cs-137 - Ba-137m.

6. Branching factors for Th227 (U235 daughter) and Cm248 (Cf252 daughter) not included in EDE equations for columns T and V. Branching factor for Pu244 (Cm248 daughter) not included in column $\mathrm{R}$.

These corrections have been made.

7. PA Tables C.3-5, C.3-9, C.3-10 and C.3-14 show that EDE contributions from daughters of Ra223 are included whereas the other PA tables (i.e., C.3-7, C.3-8, C.3-11 and C.3-12) do not. No Ra223 daughters are included in the spreadsheet. Which is correct? 
The PA tables should all indicate that the DCFs for Ra-223 include the contribution from the extremely short-lived daughters Pb-211, Bi-211 and Tl-207.

8. PA Tables C.3-9, C.3-10, C.3-14 show that EDE contributions from daughters of Pu244 (as a daughter of $\mathrm{Cm} 248$ ) are included whereas the other PA tables (i.e., C.3-5, C.3-7, C.3-8, C.3-11 and C.3-12) do not. No Pu244 daughters are included in the spreadsheet. Which is correct?

The PA tables should all indicate that the DCFs for Pu-244 include the contribution from the extremely short-lived daughter $\mathrm{Np}-240 \mathrm{~m}$, which is what is implemented in the spreadsheet.

9. Soil Ingestion EDE equation for Th234 incorrectly adds Pa234 contribution twice instead of the second daughter $(\mathrm{Pa} 234 \mathrm{~m})$.

This correction has been made.

10. General question; Why are you using the DCF for $100 \mathrm{~cm}$ shielding in calculating the Home Exposure (Resident Scenario) EDE (column V) if the basement of the home sits directly on top of the grout layer which only has only a one-foot grout envelope?

As stated in Section 6.3.2.6, the Resident Scenario is assumed to take place at 100 years, when there is still sufficient backfill that the basement will bet least $100 \mathrm{~cm}$ from the waste material. Thus the $100 \mathrm{~cm}$ shielding is correct for the Resident Scenario. Note that the Agriculture Scenario also involves a basement, and at 700 years, when this scenario is assumed to occur, the shielding is $0 \mathrm{~cm}$.

F. Columns AB, AC and AD; Summary EDEs for Agriculture, Resident and PostDrilling Scenario, respectively. Checked logic and correctness of computation and compared with Table C.3-13, 3-14 and 3-15, respectively, in PA Revision 1.

1. Basic EDE equation logic was found to be correct in all cases. Results were spot checked with hand calculation and found to be implemented correctly in the spreadsheet.

G. Columns AF, AG and AH; Fraction Remaining at 100, 300 and 700 years, respectively. Checked logic and correctness of computation and compared with Collard's Fraction remaining output from his groundwater modeling runs.

1. With the exceptions noted in the marked up spreadsheet for Pu240 and U236 all values transcribed from Collard's output is correct. Be sure to use consistent rounding rules when transferring to report tables.

Corrections made. Consistent rounding will be observed when transferring to report tables. 
2. The decay rate equation was used correctly for those radionuclides where decay was used to calculate Fraction Remaining with the exception noted in comment 3 below. Results were spot checked by hand calculation.

3. The time used in the decay equation for Fraction Remaining (i.e.,100, 300 and 700 years in columns $\mathrm{AF}, \mathrm{AG}$, and $\mathrm{AH}$, respectively) seemed to be inconsistently applied in some cases. For example in the case of Cd-113m, Sn121m, Bk249, Cf250, Cf251 and Cf252 you used 100, 700 and 1,000 years in your equation. In the case of U235 (as Pu239 daughter) you used 100, 300 and 300 years. In the case of Cm242 you used 100, 700 and 700 years.

\section{These corrections were made.}

4. General question; As I understand it $\mathrm{U}$ and $\mathrm{Pu}$ are solubility limited. In cases whe re $\mathrm{U}$ and $\mathrm{Pu}$ show up as daughters would it make sense to simply decay the parent for 100, 300 and 700 years and use that as the Fraction Remaining instead of Len's output (i.e., without leaching)?

I agree. This was implemented.

$\underline{\text { Implementation was spot checked with author. }}$

5. I need for you to explain to me the logic in those cases where you calculate the Fraction Remaining of a daughter by ratioing the half life of the parent to the daughter and then multiplying this by the Fraction Remaining of the daughter when it is a parent (i.e., starts with 1 curie) in a separate chain. See for example, Pu238 (daughter of Am242m).

The process described above corrects the fraction remaining by removing a portion of the daughter by leaching (in proportion to the amount removed when the same radionuclide is a parent).

\section{H. Columns AL, AM, AO, AP, AR and AT; CIG Limits (Ci/5 trenches and uCi/cu.m) for Agriculture, Resident and Post-Drilling Scenarios, respectively. Checked logic and correctness of computation and compared with replacement Tables 6.3-10, 6.3-16, and 6.3-20, respectively, in Special Analysis report.}

1. I need to sit down with you to understand the equation used.

The request discussion was held and the various factors explained to the reviewer's satisfaction.

The logic of these columns for calculating trench limits for each of the pathways was discussed and checked. A number of corrections were made and results spot checked with hand calculations. The limits in columns AM, AP and AS were all cross-checked with the corresponding tables in the SA and are correctly transcribed. 
I. Column AX; Final CIG Limit column (Ci/5 trenches). Checked logic and correctness of computation and compared with replacement Table 7.1-6 in Special Analysis report.

1. The limits in column AX were all cross-checked with the corresponding table in the SA and are correctly transcribed. Also, checked and verified that the proper limiting pathway was listed in SA table. 